\title{
Analysis of degenerate elliptic operators of Grušin type
}

\author{
Derek W. Robinson ${ }^{1}$ and Adam Sikora ${ }^{2}$
}

June 2006

\begin{abstract}
We analyze degenerate, second-order, elliptic operators $H$ in divergence form on $L_{2}\left(\mathbf{R}^{n} \times \mathbf{R}^{m}\right)$. We assume the coefficients are real symmetric and $a_{1} H_{\delta} \geq H \geq a_{2} H_{\delta}$ for some $a_{1}, a_{2}>0$ where$$
H_{\delta}=-\nabla_{x_{1}} c_{\delta_{1}, \delta_{1}^{\prime}}\left(x_{1}\right) \nabla_{x_{1}}-c_{\delta_{2}, \delta_{2}^{\prime}}\left(x_{1}\right) \nabla_{x_{2}}^{2}
$$

Here $x_{1} \in \mathbf{R}^{n}, x_{2} \in \mathbf{R}^{m}$ and $c_{\delta_{i}, \delta_{i}^{\prime}}$ are positive measurable functions such that $c_{\delta_{i}, \delta_{i}^{\prime}}(x)$ behaves like $|x|^{\delta_{i}}$ as $x \rightarrow 0$ and $|x|^{\delta_{i}^{\prime}}$ as $x \rightarrow \infty$ with $\delta_{1}, \delta_{1}^{\prime} \in[0,1\rangle$ and $\delta_{2}, \delta_{2}^{\prime} \geq 0$.

Our principal results state that the submarkovian semigroup $S_{t}=$ $e^{-t H}$ is conservative and its kernel $K_{t}$ satisfies bounds

$$
0 \leq K_{t}(x ; y) \leq a\left(\left|B\left(x ; t^{1 / 2}\right)\right|\left|B\left(y ; t^{1 / 2}\right)\right|\right)^{-1 / 2}
$$

where $|B(x ; r)|$ denotes the volume of the ball $B(x ; r)$ centred at $x$ with radius $r$ measured with respect to the Riemannian distance associated with $H$. The proofs depend on detailed subelliptic estimations on $H$, a precise characterization of the Riemannian distance and the corresponding volumes and wave equation techniques which exploit the finite speed of propagation.

We discuss further implications of these bounds and give explicit examples that show the kernel is not necessarily strictly positive, nor continuous.
\end{abstract}

AMS Subject Classification: 35J70, 35H20, 35L05, 58J35.

1. Mathematical Sciences Institute Australian National University Canberra, ACT 0200 Australia.
2. Department of Mathematical Sciences New Mexico State University

P.O. Box 30001

Las Cruces

NM 88003-8001, USA. 


\section{Introduction}

The classical work of Nash [Nas58, De Giorgi De 57] Aronson [Aro67] gives boundedness and regularity properties of weak solutions of linear parabolic and elliptic equations with measurable coefficients. Their results cover the equations given by second-order operators $H$ in divergence form on $L_{2}\left(\mathbf{R}^{d}\right)$, i.e., operators formally expressed as

$$
H=-\sum_{i, j=1}^{d} \partial_{i} c_{i j} \partial_{j}
$$

where $\partial_{i}=\partial / \partial x_{i}$, the $c_{i j}$ are real-valued measurable functions and the coefficient matrix $C=\left(c_{i j}\right)$ is assumed to be symmetric and positive-definite almost-everywhere. The principal hypothesis of the theory is (uniform) strong ellipticity of the coefficients. This condition is formulated as $\infty>\lambda I \geq C \geq \mu I>0$ or as the equivalent operator condition $\lambda L \geq H \geq \mu L$ where $L=-\sum_{i=1}^{d} \partial_{i}^{2}$ is the usual Laplacian. It is a condition of non-degeneracy.

The prime conclusion of the Nash-De Giorgi-Aronson theory is that the fundamental solution of the parabolic equation, the heat kernel, satisfies global Gaussian upper and lower bounds. Hölder continuity of solutions of the elliptic and parabolic equations is then a consequence of the Gaussian bounds. It has become increasingly apparent that these bounds encode a great deal of useful information on related concepts such as Riesz transforms, spectral multipliers, holomorphic functional calculus etc. (see, for example, DOS02 Ouh05 and references therein). It has also been established that the Gaussian bounds follow from two general structural features, a Poincaré inequality and volume doubling Gri92 [SC95]. This has led to extension of many results to a broader class of operators, e.g., the Laplace-Beltrami operator on Riemannian manifolds with non-negative curvature (see Gri99] and [SC02] for reviews of these developments).

The theory of degenerate elliptic operators is in comparison underdeveloped although two general classes have been identified which retain many of the structural features associated with strong ellipticity, at least locally, albeit with significant geometric modifications. These classes weaken the strong ellipticity hypotheses in different directions and cover complementary types of degeneracy but only describe some of the possibilities .

The first class consists of operators for which the largest eigenvalue $\mu_{M}$ and the inverse of the smallest eigenvalue $\mu_{m}$ of the coefficient matrix $C$ are both locally integrable [FKS82. Tru73. Poincaré and Harnack inequalities, Hölder continuity etc. follow from this condition, or slightly more stringent conditions. These conditions place direct restraints on the order of degeneracy, e.g., the order of $\mu_{m}$ in the neighbourhood of a zero, and limit the analysis to weakly degenerate operators. Under such restraints one can still exploit many of the methods used to analyze strongly elliptic operators. Note, however, that this class of operators does not contain simple examples such as the Heisenberg sublaplacian $H_{\text {Heis }}=-\partial_{1}^{2}-\left(\partial_{2}+x_{1} \partial_{3}\right)^{2}$ on $L_{2}\left(\mathbf{R}^{3}\right)$ for which $\mu_{m}$ is identically zero.

The second class consists of operators which satisfy a subelliptic estimate $H \geq \mu L^{\gamma}-\nu I$ where $\mu>0, \nu \geq 0$ and $\gamma \in\langle 0,1]$. This condition, which is a natural extension of the operator form of strong ellipticity $H \geq \mu L$, is of a rather different nature since it cannot be expressed in terms of the lowest eigenvalue $\mu_{m}$ or, indeed, in terms of the coefficients $c_{i j}$. For example, the Heisenberg sublaplacian $H_{\text {Heis }}$ on $L_{2}\left(\mathbf{R}^{3}\right)$ satisfies the estimate, with $\gamma=$ $1 / 2$. This is a specific example of a type of subelliptic operator introduced by Hörmander 
Hör67. The Hörmander operators are expressed in the form $H=\sum_{i=1}^{n} X_{i}^{*} X_{i}$ where the $X_{i}$ are smooth vector fields. The principal assumption is that the $X_{i}$ together with their multicommutators of a fixed order $r$ span the tangent space at each point $x \in \mathbf{R}^{d}$. Then $H$ satisfies the subellipticity condition locally with $\gamma=1 / r$ (see, JSC87 for a detailed review and references). The algebraic structure of the fields required by the Hörmander commutator condition places restrictions on the smoothness and growth of the coefficients of the operators. Fefferman and Phong [FP83] subsequently extended the scope of the theory by establishing that locally the subellipticity is equivalent to a property of the balls defined by the corresponding Riemannian geometry. The results of these authors then opened the way to the extension of many of the local estimates of the NashDe Giorgi-Aronson theory to the subelliptic situation (again see, [JSC87] for a detailed description). Nevertheless the results of Fefferman and Phong depend on some smoothness of the coefficients of $H$. In particular it is necessary for the coefficients to be at least twice differentiable to define the balls that play a key role in their analysis (see [FSC86], Section 1) and this is possibly sufficient. This is indeed the case in two dimensions Xu Xu92. But the $C^{2}$ requirement places a substantial restraint on the possible degeneracy.

In this paper we introduce and analyze global properties of a family of degenerate operators with measurable coefficients which is not covered by either of the above classes but which incorporates two different types of degeneracy typical of the classes. As a preliminary we define two functions $f, g$ with values in an ordered space to be equivalent, $f \sim g$, if there are $a, a^{\prime}>0$ such that $a f \leq g \leq a^{\prime} f$ uniformly. We use this notion in a variety of contexts, e.g., for functions over $\mathbf{R}^{d}$, for positive symmetric matrices and lower semibounded selfadjoint operators and also for the equivalence of quadratic forms. In addition for $a>0$ we set

$$
a^{\left(\alpha, \alpha^{\prime}\right)}= \begin{cases}a^{\alpha} & \text { if } a \leq 1 \\ a^{\alpha^{\prime}} & \text { if } a \geq 1\end{cases}
$$

for all $\alpha, \alpha^{\prime} \in \mathbf{R}$.

We analyze operators $H$ on $L_{2}\left(\mathbf{R}^{n} \times \mathbf{R}^{m}\right)$ of the form (11) but with the matrix of coefficients $C \sim C_{\delta}$ where $C_{\delta}$ is a block diagonal matrix $C_{\delta}\left(x_{1}, x_{2}\right)=c_{\delta_{1}, \delta_{1}^{\prime}}\left(x_{1}\right) I_{n}+c_{\delta_{2}, \delta_{2}^{\prime}}\left(x_{1}\right) I_{m}$ and $c_{\delta_{1}, \delta_{1}^{\prime}}, c_{\delta_{2}, \delta_{2}^{\prime}}$ are positive measurable functions such that

$$
c_{\delta_{i}, \delta_{i}^{\prime}}(x) \sim|x|^{\left(2 \delta_{i}, 2 \delta_{i}^{\prime}\right)}
$$

where the indices $\delta_{1}, \delta_{2}, \delta_{1}^{\prime}, \delta_{2}^{\prime}$ are all non-negative and $\delta_{1}, \delta_{1}^{\prime}<1$ but there is no upper bound on $\delta_{2}$ and $\delta_{2}^{\prime}$. Thus $H \sim H_{\delta}$, where $H_{\delta}$ is given by

$$
H_{\delta}=-\nabla_{x_{1}} c_{\delta_{1}, \delta_{1}^{\prime}} \nabla_{x_{1}}+c_{\delta_{2}, \delta_{2}^{\prime}} L_{x_{2}}
$$

with $x_{1} \in \mathbf{R}^{n}, x_{2} \in \mathbf{R}^{m}, \nabla_{x_{1}}$ the gradient operator on $L_{2}\left(\mathbf{R}^{n}\right)$ and $L_{x_{2}}=-\nabla_{x_{2}}^{2}$ the Laplacian on $L_{2}\left(\mathbf{R}^{m}\right)$. Since $C_{\delta}$ is only defined up to equivalence there is a freedom of choice which will often be exploited in the sequel.

In summary we consider elliptic operators $H$ of the form (11), defined precisely by quadratic form techniques in Section 2, with $H \sim H_{\delta}$ where $H_{\delta}$ is the elliptic operator (3) with coefficients satisfying (21). The operator (3) is a natural generalization of the Hörmander type operators $-\partial_{1}^{2}-x_{1}^{2 n} \partial_{2}^{2}$ introduced by Grušin Gru70]. Therefore we refer to them as Grušin operators. Note that for these operators the lowest eigenvalue $\mu_{m}$ of 
the coefficient matrix $C$ satisfies $\mu_{m}(x) \sim\left|x_{1}\right|^{2\left(\delta_{1} \vee \delta_{2}\right)}$ for $|x| \leq 1$ thus the inverse of $\mu_{m}$ is locally integrable if and only if $\delta_{1} \vee \delta_{2}<n$. Moreover, we make no smoothness assumptions on the coefficients $c_{i j}$.

We will prove that the semigroup $S$ generated by the Grušin operator $H$ is conservative, i.e., $S_{t} \mathbb{1}=\mathbb{1}$ on $L_{\infty}\left(\mathbf{R}^{n+m}\right)$ and that its kernel $K$ satisfies Gaussian bounds with respect to the appropriate Riemannian geometry.

The Riemannian distance associated with an elliptic operator (11) with measurable coefficients $C=\left(c_{i j}\right)$ is defined by

$$
d(y ; z)=\sup _{\psi \in D}(\psi(y)-\psi(z))
$$

for all $y, z \in \mathbf{R}^{d}$ where

$$
D=\left\{\psi \in W^{1, \infty}\left(\mathbf{R}^{d}\right): \sum_{i, j=1}^{d} c_{i j}\left(\partial_{i} \psi\right)\left(\partial_{j} \psi\right) \leq 1\right\}
$$

If $C$ is strongly elliptic then this is equivalent to the usual Euclidean distance but for a general elliptic operator it is not necessarily a genuine distance, i.e., it could take the value infinity. If, however, $H$ is a Grušin operator on $\mathbf{R}^{n} \times \mathbf{R}^{m}$ then $C \sim C_{\delta}$ and $d(\cdot ; \cdot)$ is equivalent to

$d_{\delta}(y ; z)=\sup \left\{|\psi(y)-\psi(z)|: \psi \in W^{1, \infty}\left(\mathbf{R}^{n} \times \mathbf{R}^{m}\right), c_{\delta_{1}, \delta_{1}^{\prime}}\left|\nabla_{x_{1}} \psi\right|^{2}+c_{\delta_{2}, \delta_{2}^{\prime}}\left|\nabla_{x_{2}} \psi\right|^{2} \leq 1\right\}$.

The restrictions $\delta_{1}, \delta_{1}^{\prime}<1$ ensure that this latter function is a bona fide distance.

There are two technical difficulties in our analysis of Grušin operators. First one needs to derive subelliptic estimates on $H_{\delta}$ in order to obtain a priori estimates on the semigroup $S$ (see Section 31). Secondly it is necessary to estimate the volume (Lebesgue measure) $|B(x ; r)|$ of the Riemannian balls $B(x ; r)=\{y: d(x ; y)<r\}$ or, equivalently, the balls $B_{\delta}(x ; r)=\left\{y: d_{\delta}(x ; y)<r\right\}$ (see Section [5). But then we establish in Section 6 that the semigroup $S$ is conservative and and its kernel $K$ satisfies Gaussian bounds. Specifically, for each $\varepsilon \in\langle 0,1]$ there is an $a>0$ such that

$$
0 \leq K_{t}(x ; y) \leq a\left(\left|B\left(x ; t^{1 / 2}\right)\right|\left|B\left(y ; t^{1 / 2}\right)\right|\right)^{-1 / 2} e^{-d(x ; y)^{2} /(4(1+\varepsilon) t)}
$$

for all $x, y \in \mathbf{R}^{n} \times \mathbf{R}^{m}$ and all $t>0$.

Lower bounds and continuity properties are more sensitive, e.g., if $n=1$ and $\delta_{1} \in$ $[1 / 2,1\rangle$ then the kernel is not strictly positive nor is it continuous (see [ERSZ06], Sections 5 and 6) since the system separates into two distinct subsystems, the halfspace $x_{1} \geq 0$ and the halfspace $x_{1} \leq 0$. In Section [7 we discuss the simplest example in one-dimension, $n=1$, $m=0$. The one-dimensional example has been analyzed at length in the setting of control theory ABCF06 [MV06] from a slightly different perspective. In the latter context the case $\delta \in[0,1 / 2\rangle$ is referred to as weakly degenerate and the case $\delta_{1} \in[1 / 2,1\rangle$ as strongly degenerate. We will indeed establish positivity and continuity properties similar to those associated with strongly elliptic operators in the weakly degenerate case. In addition we establish some partial regularity and positivity properties for the ergodic components on $L_{2}\left(\mathbf{R}_{ \pm}\right)$in the strongly degenerate case. We emphasize that the separation phenomenon demonstrates that the Riemannian distance is not appropriate for the full description of the structure associated with strongly degenerate operators. Indeed the small time asymptotics 
of the kernel is given by a larger distance [ERS06] HR03] AH05] which incorporates the separation. This indicates that the above Gaussian bounds are not optimal. Nevertheless, they suffice for the derivation of several significant results in the global spectral analysis of Grušin operators (see Section 8).

Finally we note that Sawyer and Wheeden [SW06] have recently reformulated the Hörmander-Fefferman-Phong theory to incorporate elliptic operators with $L_{\infty}$-coefficients. But their emphasis is from the outset on establishing local regularity properties of weak solutions. Our analysis of Grušin operators with $n=1$ and $\delta_{1} \in[1 / 2,1\rangle$ shows that such properties are not universally valid.

\section{Preliminaries}

In the sequel we are only interested in qualitative estimates. Hence we adopt the variable constant convention. In subsequent estimates $a, a^{\prime}$ etc. denote strictly positive constants whose value may vary bound by bound but which are independent of the key variables in the estimates.

We begin by discussing the precise definition of elliptic operators of the form (11) or more specifically (3) through closed quadratic forms.

First, let $C=\left(c_{i j}\right)$ be a real-valued symmetric matrix with measurable, locally integrable, coefficients $c_{i j}$. Assume $C$ is positive-definite almost everywhere and define the positive quadratic form $h$ by

$$
h(\varphi)=\sum_{i, j=1}^{d}\left(\partial_{i} \varphi, c_{i j} \partial_{j} \varphi\right)=\int_{\mathbf{R}^{d}} d x \sum_{i, j=1}^{d} c_{i j}(x)\left(\partial_{i} \varphi\right)(x)\left(\partial_{j} \varphi\right)(x)
$$

with $D(h)$ consisting of those $\varphi \in W^{1,2}\left(\mathbf{R}^{d}\right)$ for which the integral converges. Since the coefficients are locally integrable the form is densely defined. But it is not necessarily closed. There are three possibilities.

First, if $h$ is closed then there is a positive self-adjoint operator $H$ such that $D(h)=$ $D\left(H^{1 / 2}\right)$ and $h(\varphi)=\left\|H^{1 / 2} \varphi\right\|_{2}^{2}$. Then we define $H$ to be the elliptic operator with coefficients $C$. For example if $C$ satisfies the usual strong ellipticity assumptions, $\lambda I \geq C \geq$ $\mu I>0$, then $D(h)=W^{1,2}\left(\mathbf{R}^{d}\right)$ and $h$ is closed. More generally one has the following.

Lemma 2.1 If $C \geq \mu I>0$ almost everywhere then $h$ is closed.

Proof The lemma is established by a monotonicity argument. If $C_{N}=C \wedge N I$ with $N \in \mathbf{N}$ then $C_{N}$ is strongly elliptic and the corresponding closed forms $h_{N}$ with $D\left(h_{N}\right)=$ $W^{1,2}\left(\mathbf{R}^{d}\right)$ are monotonically increasing. But $h(\varphi)=\sup _{N} h_{N}(\varphi)$ with $D(h)$ the subspace of $W^{1,2}\left(\mathbf{R}^{d}\right)$ for which the supremum is finite. Then, however, $h$ is closed (for details see, for example [BR97, Lemma 5.2.23).

Remark 2.2 Note that if $H$ is the elliptic operator associated with the closed form $h$ in the lemma and $H_{N}$ the strongly elliptic operators associated with the $h_{N}$ then the $H_{N}$ converge in the strong resolvent sense to $H$. But the $h_{N}$ are Dirichlet forms. Hence $h$ is a Dirichlet form and the semigroup $S$ generated by $H$ is submarkovian. 
Secondly, it is possible that $h$ is closable although it is not closed. Then the elliptic operator $H$ is naturally defined as the self-adjoint operator associated with the closure $\bar{h}$ of $h$. Again $\bar{h}$ is a Dirichlet form and the elliptic semigroup $S$ is submarkovian. This is indeed the case of principal interest in the current context.

Lemma 2.3 The form $h$ of a Grušin operator is closable.

Proof Let $h_{\delta}$ denote the form of the operator $H_{\delta}$ given formally by (3). Since $C \sim C_{\delta}$ it follows that $h \sim h_{\delta}$. Explicitly, $D(h)=D\left(h_{\delta}\right)$ and there are $a, a^{\prime}>0$ such that

$$
a h_{\delta}(\varphi) \geq h(\varphi) \geq a^{\prime} h_{\delta}(\varphi)
$$

for all $\varphi \in D(h)$. Thus it suffices to prove that $h_{\delta}$ is closable. But we may assume the coefficients $c_{\delta_{1}, \delta_{1}^{\prime}}, c_{\delta_{2}, \delta_{2}^{\prime}}$ are continuous. Then $h_{\delta}$ is closable by the proof of Proposition 2.3 in [ERSZ06].

Although the forms of the Grušin operators are closable it is nevertheless of interest to consider the third possibility for general elliptic forms, the possibility that $h$ is neither closed nor closable. (For explicit examples see [FOT94 Section 3.1 and in particular Theorem 3.1). In this case one can introduce the relaxation $h_{0}$ of $h$ as the largest positive, closed, quadratic form $h_{0}$ such that $h_{0} \leq h$. The relaxation occurs in the context of nonlinear phenomena and discontinuous media (see, for example, Bra02] [ET76] [Jos98] Dal93 Mos94 and references therein) and can be characterized in several different ways. Simon [Sim78, Theorems 2.1 and 2.2, has shown that a general positive quadratic form $h$ can be decomposed as a sum $h=h_{r}+h_{s}$ of two positive forms with $D\left(h_{r}\right)=D(h)=D\left(h_{s}\right)$ with $h_{r}$ the largest closable form majorized by $h$. Simon refers to $h_{r}$ as the regular part of $h$. Then $h_{0}=\bar{h}_{r}$. Note that $h_{0}=h$ if $h$ is closed and $h_{0}=\bar{h}$ if $h$ is closable.

There is an alternative method of constructing $h_{0}$ by monotone approximation. Let $l$ be the closed quadratic form associated with the Laplacian $L$. Then $D(l)=W^{1,2}\left(\mathbf{R}^{d}\right)$. Next define $h_{\varepsilon}=h+\varepsilon l$ for $\varepsilon>0$ with $D\left(h_{\varepsilon}\right)=D(h)$. Then $h_{\varepsilon}$ corresponds to the elliptic operator with coefficients $c_{i j}+\varepsilon \delta_{i j}$ and is closed by Lemma 2.1. The corresponding positive self-adjoint operators $H_{\varepsilon}$ form a decreasing sequence which, by a result of Kato Kat80, Theorem VIII.3.11, converges in the strong resolvent sense to a positive self-adjoint operator $H_{0}$. This is the operator $H_{0}$ associated with the relaxation $h_{0}$ (see Sim78, Theorem 3.2). The latter construction justifies the interpretation of $H_{0}$ as the elliptic operator with coefficients $C$. It follows again that the relaxation $h_{0}$ is a Dirichlet form. Moreover the construction of the relaxation respects order properties, i.e., if $h$ and $k$ are two elliptic forms and $h \geq k$ then $h_{0} \geq k_{0}$.

In each of the above situations the elliptic operator $H$ with coefficients $C$ is obtained as a double limit $N \rightarrow \infty, \varepsilon \rightarrow 0$ of the strongly elliptic operators $H_{N, \varepsilon}$ with coefficients $C_{N, \varepsilon}=(C \wedge N I)+\varepsilon I$. The convergence is in the strong resolvent sense. In particular this construction is applicable to the Grušin operators. Therefore it is not essential to make a notational distinction between the three cases. Thus in the sequel we use $H$ to denote the self-adjoint elliptic operator constructed by this limiting process, $S$ the submarkovian semigroup generated by $H$ and $K$ the semigroup kernel. It follows in particular from the construction that the semigroups $S^{(N, \varepsilon)}$ generated by the strongly elliptic operators $H_{N, \varepsilon}$ converge strongly to $S$ on $L_{2}\left(\mathbf{R}^{d}\right)$. 
Our next aim is to discuss a priori bounds on $t \rightarrow K_{t}$ which are uniform over $\mathbf{R}^{d}$. This is equivalent to obtaining bounds on the crossnorms $\left\|S_{t}\right\|_{1 \rightarrow \infty}$ of the semigroup as a map from $L_{1}$ to $L_{\infty}$, or the crossnorms $\left\|S_{t}\right\|_{2 \rightarrow \infty}$ from $L_{2}$ to $L_{\infty}$ since

$$
\left\|S_{t}\right\|_{1 \rightarrow \infty}=\operatorname{esssup}_{x, y \in \mathbf{R}^{d}}\left|K_{t}(x ; y)\right|=\operatorname{essiup}_{x \in \mathbf{R}^{d}} \int_{\mathbf{R}^{d}} d y\left|K_{t / 2}(x ; y)\right|^{2}=\left(\left\|S_{t / 2}\right\|_{2 \rightarrow \infty}\right)^{2}
$$

for all $t>0$.

The standard method of obtaining bounds on the crossnorms $\left\|S_{t}\right\|_{2 \rightarrow \infty}$, and hence on $\left\|S_{t}\right\|_{1 \rightarrow \infty}$, for strongly elliptic semigroups is via Nash inequalities. In the context of degenerate elliptic operators it is useful to consider a particular class of inequalities defined in terms of Fourier multipliers.

Let $F$ be a positive real function over $\mathbf{R}^{d}$ and define the corresponding Fourier multiplier $F$ on $L_{2}\left(\mathbf{R}^{d}\right)$ by $\widetilde{(F \varphi)}(p)=F(p) \widetilde{\varphi}(p)$ where $\widetilde{\varphi}$ denotes the Fourier transform of $\varphi \in L_{2}\left(\mathbf{R}^{d}\right)$. One can also interpret $F$ as a differential operator $F=F\left(i \nabla_{x}\right)$ with constant coefficients. Next let $f$ denote the closed form corresponding to $F$, i.e.,

$$
f(\varphi)=\int_{\mathbf{R}^{d}} d p F(p)|\widetilde{\varphi}(p)|^{2}
$$

with $D(f)$ the subspace of $\varphi \in L_{2}\left(\mathbf{R}^{d}\right)$ for which the integral is finite. Finally let $V_{F}(r)$ denote the volume (Lebesgue measure) of the set $\left\{p: F(p)<r^{2}\right\}$.

A subelliptic estimate of the form $h \geq f$ immediately gives a Nash type inequality.

Lemma 2.4 If $h \geq f$ then

$$
\|\varphi\|_{2}^{2} \leq r^{-2} h(\varphi)+(2 \pi)^{-d} V_{F}(r)\|\varphi\|_{1}^{2}
$$

for all $\varphi \in D(h) \cap L_{1}$ and all $r>0$.

Proof The proof is a direct consequence of the Plancherel formula;

$$
\begin{aligned}
\|\varphi\|_{2}^{2}=\int_{\mathbf{R}^{d}} d p|\widetilde{\varphi}(p)|^{2} & =\int_{F(p) \geq r^{2}} d p|\widetilde{\varphi}(p)|^{2}+\int_{F(p)<r^{2}} d p|\widetilde{\varphi}(p)|^{2} \\
& \leq r^{-2} \int_{\mathbf{R}^{d}} d p F(p)|\widetilde{\varphi}(p)|^{2}+(2 \pi)^{-d} \int_{F(p)<r^{2}} d p\|\varphi\|_{1}^{2} \\
& \leq r^{-2} h(\varphi)+(2 \pi)^{-d} V_{F}(r)\|\varphi\|_{1}^{2}
\end{aligned}
$$

for all $r>0$.

The Nash inequality allows one to obtain bounds on the cross-norm $\left\|S_{t}\right\|_{2 \rightarrow \infty}$ for many different $F$. In particular if $V_{F}$ has a polynomial behaviour one can estimate $\left\|S_{t}\right\|_{1 \rightarrow 2}$, and by duality $\left\|S_{t}\right\|_{2 \rightarrow \infty}$, by a straightforward extension of Nash's original argument. In particular one obtains the following.

Lemma 2.5 If $h \geq f$ and $V_{F}(r) \leq a r^{\left(D^{\prime}, D\right)}$ then $\left\|S_{t}\right\|_{1 \rightarrow \infty} \leq b t^{\left(-D / 2,-D^{\prime} / 2\right)}$. 
Proof The result can be deduced from [CKS87, Theorem 2.9 or from the alternative argument given in Rob91 pages 268-269.

The lemma demonstrates that large values of $r$ give small $t$ bounds and small values of $r$ give large $t$ bounds. If $h$ is strongly elliptic then $F(p)=\mu p^{2}=\left(\mu^{1 / 2} p\right)^{2}, V_{F}(r) \sim r^{d}$ and $\left\|S_{t}\right\|_{1 \rightarrow \infty} \leq a(\mu t)^{-d / 2}$ for all $t>0$. Alternatively the subellipticity condition $H \geq \mu L^{\gamma}-\nu I$ corresponds to $F(p)=\left(\mu|p|^{2 \gamma}-\nu\right) \vee 0$ and this only gives useful information on the large $r$ behaviour of $V_{F}$. It yields bounds $\left\|S_{t}\right\|_{1 \rightarrow \infty} \leq a t^{-d /(2 \gamma)}$ for $t \leq 1$.

Lemmas 2.4 and 2.5 allow us in Section 3 to obtain uniform bounds on the semigroup kernels associated with the Grušin operators (3) except if $n=1$ and $\delta_{1}$ or $\delta_{2}$ is in $[1 / 2,1\rangle$. In the latter case one obtains subelliptic bounds of a different character, bounds in terms of the Neumann Laplacian. But these can also be used to obtain Nash inequalities.

Let $L_{x, N}$ denote the self-adjoint version of the operator $-d^{2} / d x^{2}$ on $L_{2}(\mathbf{R})$ with Neumann boundary conditions at the origin. Then $L_{x, N}=L_{x, N}^{+} \oplus L_{x, N}^{+}$where $L_{x, N}^{ \pm}$are the Neumann operators on $L_{2}\left(\mathbf{R}_{ \pm}\right)$, respectively. Now let $F$ be a positive function on the half-line and define the operators $F_{N}=F\left(L_{x, N}\right)$ and $F_{N}^{ \pm}=F\left(L_{x, N}^{ \pm}\right)$by spectral theory. Then $F_{N}=F_{N}^{+} \oplus F_{N}^{-}$. Next let $f_{N}$ and $f_{N}^{ \pm}$be the forms corresponding to $F_{N}$ and $F_{N}^{ \pm}$. Each $\varphi \in D\left(f_{N}\right)$ has a unique decomposition $\varphi=\varphi_{+} \oplus \varphi_{-}$with $\varphi_{ \pm} \in D\left(f_{N}^{ \pm}\right)$and one has the relations $\|\varphi\|_{2}^{2}=\left\|\varphi_{+}\right\|_{2}^{2}+\left\|\varphi_{-}\right\|_{2}^{2}, f_{N}(\varphi)=f_{N}^{+}\left(\varphi_{+}\right)+f_{N}^{-}\left(\varphi_{-}\right)$and $\|\varphi\|_{1}=\left\|\varphi_{+}\right\|_{1}+\left\|\varphi_{-}\right\|_{1}$. Thus the Nash inequalities can be analyzed by examining the two subsystems on $L_{2}\left(\mathbf{R}_{ \pm}\right)$. This can then be handled by considering the extension of the operators from $L_{2}\left(\mathbf{R}_{ \pm}\right)$to the space $L_{2, e}(\mathbf{R})$ of even functions over the line. Since both cases are similar we only consider the extension of $L_{2}\left(\mathbf{R}_{+}\right)$.

First, if $\varphi \in L_{2}\left(\mathbf{R}_{+}\right)$we define the symmetric extension $E \varphi$ to $L_{2}(\mathbf{R})$ by $(E \varphi)( \pm x)=$ $\varphi(x)$ if $x \geq 0$. Then $L_{2, e}(\mathbf{R})$ is the range of $E$. Secondly, if $\varphi \in L_{2}(\mathbf{R})$ we define the restriction $R \varphi$ to $L_{2}\left(\mathbf{R}_{+}\right)$by $(R \varphi)(x)=\varphi(x)$ if $x \geq 0$. Thirdly, if $T$ denotes the semigroup generated by the one-dimensional Laplacian $L_{x}=-d^{2} / d x^{2}$ on $L_{2}(\mathbf{R})$ then $T$ leaves $L_{2, e}(\mathbf{R})$ invariant. Hence $R T E$ defines a semigroup on $L_{2}\left(\mathbf{R}_{+}\right)$. The semigroup property follows because

$$
\left(R T_{s} E\right)\left(R T_{t} E\right)=\left(R T_{s}\right)(E R)\left(T_{t} E\right)=\left(R T_{s}\right)\left(T_{t} E\right)=\left(R T_{s+t} E\right)
$$

where the second step uses the invariance. Now it is easy to check that $R T_{t} E=T_{t}^{(N)}$ where $T^{(N)}$ denotes the semigroup generated by $L_{x, N}$. Similar considerations apply to $L_{x_{1}, N} \otimes \mathbb{1}$ on $L_{2}\left(\mathbf{R} \times \mathbf{R}^{m}\right)$. If $E \varphi$ is the even extension of $\varphi \in L_{2}\left(\mathbf{R}_{+} \times \mathbf{R}^{m}\right)$ to $L_{2}\left(\mathbf{R} \times \mathbf{R}^{m}\right)$ and $R$ the corresponding restriction then one has $R\left(T_{t} \otimes \mathbb{1}\right) E=T_{t}^{(N)} \otimes \mathbb{1}$.

Next let $F$ be a positive bounded function on $\mathbf{R}_{+} \times \mathbf{R}^{m}$. Define the multiplier by $F$ by $\widetilde{(F \varphi)}\left(p_{1}, p_{2}\right)=F\left(p_{1}^{2}, p_{2}\right) \widetilde{\varphi}\left(p_{1}, p_{2}\right)$ and the corresponding operators on $L_{2}\left(\mathbf{R} \times \mathbf{R}^{m}\right)$ by $F=F\left(L_{x_{1}}, i \nabla_{x_{2}}\right)$ and $F_{N}=F\left(L_{x_{1}, N}, i \nabla_{x_{2}}\right)$. Let $F_{N}^{+}$denote the restriction of $F_{N}$ to $L_{2}\left(\mathbf{R}_{+} \times \mathbf{R}^{m}\right)$. Further let $f, f_{N}$ and $f_{N}^{+}$be the corresponding forms. Then if $\varphi \in D\left(f_{N}^{+}\right)$ one has $E \varphi \in D(f)$ and

$$
f(E \varphi)=(E \varphi, F E \varphi)=(E \varphi, E R F E \varphi)=\left(E \varphi, E F_{N}^{+} \varphi\right)=2\left(\varphi, F_{N}^{+} \varphi\right)=2 f_{N}^{+}(\varphi) .
$$

Hence using the estimate (8) for $F$ one deduces that

$$
\|\varphi\|_{2}^{2}=2^{-1}\|E \varphi\|_{2}^{2} \leq 2^{-1} r^{-2}(E \varphi, F E \varphi)+2^{-1} V_{F}(r)\|E \varphi\|_{1}^{2}=r^{-2} f_{N}^{+}(\varphi)+2(2 \pi)^{-d} V_{F}(r)\|\varphi\|_{1}^{2}
$$

for all $r>0$. 
One can reason similarly for the restriction to the left half line and then by combination one obtains the following analogue of Lemma 2.4 with $V_{F}(r)$ the volume of the set $\left\{\left(p_{1}, p_{2}\right)\right.$ : $\left.F\left(p_{1}^{2}, p_{2}\right)<r^{2}\right\}$.

Lemma 2.6 If $h \geq f_{N}$ in the form sense on $L_{2}\left(\mathbf{R} \times \mathbf{R}^{m}\right)$ then

$$
\|\varphi\|_{2}^{2} \leq r^{-2} h(\varphi)+4(2 \pi)^{-d} V_{F}(r)\|\varphi\|_{1}^{2}
$$

for all $\varphi \in D(h) \cap L_{1}\left(\mathbf{R} \times \mathbf{R}^{m}\right)$ and all $r>0$.

If the function $V_{F}$ in the Nash inequality of Lemma 2.6 has a polynomial growth of the type considered in Lemma 2.5 one then obtains analogous bounds on the crossnorm of the semigroup $S_{t}$. Thus the key point in the analysis of the Grušin operator with $n=1$ is to obtain a subelliptic estimate $h \geq f_{N}$ with the modified Fourier multiplier involving the Neumann Laplacian in the first direction.

\section{Subelliptic estimates}

In this section we examine the Grušin operators and derive uniform estimates on the semigroup crossnorms $\left\|S_{t}\right\|_{1 \rightarrow \infty}$ by use of the Nash inequalities of Lemmas [2.4 and 2.6. These bounds will then be improved by other techniques in the sequel. The principal result of this section is given by the following proposition.

Proposition 3.1 Let $S_{t}$ denote the positive self-adjoint semigroup on $L_{2}\left(\mathbf{R}^{n} \times \mathbf{R}^{m}\right)$ generated by the Grušin operator $H$ with coefficients $C \sim C_{\delta}$ where $C_{\delta}$ satisfies (2). Then

$$
\left\|S_{t}\right\|_{1 \rightarrow \infty} \leq a t^{\left(-D / 2,-D^{\prime} / 2\right)}
$$

where

$$
D=\left(n+m\left(1+\delta_{2}-\delta_{1}\right)\right)\left(1-\delta_{1}\right)^{-1} \quad \text { and } \quad D^{\prime}=\left(n+m\left(1+\delta_{2}^{\prime}-\delta_{1}^{\prime}\right)\right)\left(1-\delta_{1}^{\prime}\right)^{-1} .
$$

Note that the local dimension $D$ depends only on the parameters $\delta_{1}$ and $\delta_{2}$ which govern the local degeneracies of the coefficients of $H$ and the global dimension $D^{\prime}$ depends on the parameters $\delta_{1}^{\prime}$ and $\delta_{2}^{\prime}$ which govern the global degeneracies. Moreover, $D, D^{\prime} \geq n+m$, the Euclidean dimension, with $D=n+m$ if and only if $\delta_{1}=0=\delta_{2}$ and $D^{\prime}=n+m$ if and only if $\delta_{1}^{\prime}=0=\delta_{2}^{\prime}$.

The proof of the proposition is in two stages. First consider the operator

$$
H_{1}=-\nabla_{x} c_{\delta_{1}, \delta_{1}^{\prime}} \nabla_{x}
$$

on $L_{2}\left(\mathbf{R}^{n}\right)$. There are two possibilities.

Either $\delta_{1} \geq \delta_{1}^{\prime}$ and then $c_{\delta_{1}, \delta_{1}^{\prime}}(x) \sim|x|^{2 \delta_{1}}\left(1+|x|^{2 \delta_{1}}\right)^{-1+\delta_{1}^{\prime} / \delta_{1}}$,

or $\quad \delta_{1} \leq \delta_{1}^{\prime}$ and then $c_{\delta_{1}, \delta_{1}^{\prime}}(x) \sim|x|^{2 \delta_{1}}+|x|^{2 \delta_{1}^{\prime}}$.

Since the subsequent estimates are valid up to equivalence we can effectively replace $c_{\delta_{1}, \delta_{1}^{\prime}}$ by the appropriate function on the right. Moreover, the form

$$
h_{1}(\varphi)=\left(\nabla_{x} \varphi, c_{\delta_{1}, \delta_{1}^{\prime}} \nabla_{x} \varphi\right)=\int_{\mathbf{R}^{n}} d x c_{\delta_{1}, \delta_{1}^{\prime}}(x)\left|\left(\nabla_{x} \varphi\right)(x)\right|^{2}
$$


is closed on the domain $D\left(h_{1}\right)=W^{1,2}\left(\mathbf{R}^{n} ; c_{\delta_{1}, \delta_{1}^{\prime}} d x\right)$ and $C_{c}^{\infty}\left(\mathbf{R}^{n}\right)$ is a core of $h$. Therefore it suffices to establish the following form estimates on $C_{c}^{\infty}\left(\mathbf{R}^{n}\right)$.

It is convenient to express the subelliptic estimates in operator terms. Recall that $L_{x}=-\nabla_{x}^{2}$ denotes the Laplacian.

Proposition 3.2 Let $n \geq 2$, or $n=1$ and $\delta_{1}, \delta_{1}^{\prime} \in[0,1 / 2\rangle$. Then $h_{1} \geq f$ on $L_{2}\left(\mathbf{R}^{n} \times \mathbf{R}^{m}\right)$ where $f$ is the form of the operator $F$ with

$$
F \sim L_{x}^{\left(1-\delta_{1}^{\prime}\right)}\left(1+L_{x}\right)^{-\left(\delta_{1}-\delta_{1}^{\prime}\right)}
$$

if $\delta_{1} \geq \delta_{1}^{\prime}$ and

$$
F \sim L_{x}^{\left(1-\delta_{1}\right)}+L_{x}^{\left(1-\delta_{1}^{\prime}\right)}
$$

if $\delta_{1} \leq \delta_{1}^{\prime}$.

Moreover, if $n=1$ and $\delta_{1} \in[1 / 2,1\rangle$ or $\delta_{1}^{\prime} \in[1 / 2,1\rangle$ then $h_{1} \geq f_{N}$ where $f_{N}$ is the form of the operator $F_{N}$ obtained by replacing $L_{x}$ by $L_{x, N}$.

The two key properties needed for subelliptic estimates of $H_{1}$ are the following.

Lemma 3.3 If $\gamma \in[0,1 \wedge n / 2\rangle$ then

$$
L_{x}^{\gamma} \geq a|x|^{-2 \gamma}
$$

in the form sense on $L_{2}\left(\mathbf{R}^{n}\right)$.

Moreover, if $n=1$ then $L_{x, D} \geq\left(4 x^{2}\right)^{-1}$ on $L_{2}(\mathbf{R})$ where $L_{x, D}$ is the Laplacian with Dirichlet boundary conditions at the origin.

The statements are versions of Hardy's inequality (see, for example, Dav99 and references therein) and special cases of the inequalities of Caffarelli, Kohn and Nirenberg CKN84. The multidimensional version is often stated with $\gamma=1$ and $n \geq 3$. In the latter case one has $a=(n-2)^{2} / 4$ and this value is optimal. The fractional version follows from Strichartz' work [Str67] on Fourier multipliers.

Lemma 3.4 Let $A$ and $B$ be self-adjoint operators on $L_{2}\left(\mathbf{R}^{n}\right)$ and let $\gamma \in[0,1]$. If $A \geq$ $B \geq 0$ then

$$
A(I+A)^{-\gamma} \geq B(I+B)^{-\gamma}
$$

in the form sense.

Proof First one has

$$
A(\lambda I+A)^{-1}=I-\lambda(\lambda I+A)^{-1} \geq I-\lambda(\lambda I+B)^{-1}=B(\lambda I+B)^{-1}
$$

for all $\lambda>0$ and this gives the result for $\gamma=1$. But if $\gamma<1$ then

$$
\begin{aligned}
A(I+A)^{-\gamma} & =c_{\gamma} \int_{0}^{\infty} \frac{d \lambda}{\lambda^{\gamma}} A((1+\lambda) I+A)^{-1} \\
& \geq c_{\gamma} \int_{0}^{\infty} \frac{d \lambda}{\lambda^{\gamma}} B((1+\lambda) I+B)^{-1}=B(I+B)^{-\gamma}
\end{aligned}
$$

where we have used the standard integral representation of the fractional power. 
Proof of Proposition 3.2 Consider the case $\delta_{1} \geq \delta_{1}^{\prime}$. Then for $\varphi \in C_{c}^{\infty}\left(\mathbf{R}^{n}\right)$

$$
\begin{aligned}
h_{1}(\varphi) & \geq a\left(\nabla_{x} \varphi,|x|^{2 \delta_{1}}\left(1+|x|^{2 \delta_{1}}\right)^{-1+\delta_{1}^{\prime} / \delta_{1}} \nabla_{x} \varphi\right) \\
& \geq a\left(\nabla_{x} \varphi, L_{x}^{-\delta_{1}}\left(1+L_{x}^{-\delta_{1}}\right)^{-1+\delta_{1}^{\prime} / \delta_{1}} \nabla_{x} \varphi\right) \geq a\left(\varphi, L_{x}^{1-\delta_{1}^{\prime}}\left(1+L_{x}\right)^{-\left(\delta_{1}-\delta_{1}^{\prime}\right)} \varphi\right)
\end{aligned}
$$

by Lemmas 3.3 and 3.4 which are applicable if $\delta_{1} \in[0,1 \wedge n / 2\rangle$ and $\delta_{1}^{\prime} / \delta_{1} \in[0,1\rangle$. If, however, $n=1, \delta_{1} \in[1 / 2,1\rangle$ and $\varphi \in C_{c}^{\infty}\left(\mathbf{R}^{n} \backslash\{0\}\right)$ then

$$
h_{1}(\varphi) \geq a\left(\varphi^{\prime}, L_{x, D}^{-\delta_{1}}\left(1+L_{x, D}^{-\delta_{1}}\right)^{-1+\delta_{1}^{\prime} / \delta_{1}} \varphi^{\prime}\right) \geq a\left(\varphi, L_{x, N}^{1-\delta_{1}^{\prime}}\left(1+L_{x, N}\right)^{-\left(\delta_{1}-\delta_{1}^{\prime}\right)} \varphi\right)
$$

where the second bound follows from the argument given in Example 6.7 of ERSZ06.

The case $\delta_{1} \leq \delta_{1}^{\prime}$ is similar but simpler. It also uses the basic inequality $L_{x} \geq L_{x, N}$ which then extends to all fractional powers.

Next consider the Grušin operator (3) on $L_{2}\left(\mathbf{R}^{n} \times \mathbf{R}^{m}\right)$ and let $h$ denote the corresponding quadratic form. The $\delta$ and $\delta^{\prime}$ are positive and $\delta_{1}, \delta_{1}^{\prime} \in[0,1\rangle$ but we do not place any restriction on $\delta_{2}$ and $\delta_{2}^{\prime}$. Clearly $H \geq a H_{1}\left(=a H_{1} \otimes \mathbb{1}\right)$ and so the bounds of Proposition 3.2 are applicable. But then one has the following complementary bounds.

Proposition 3.5 The subelliptic estimate $h \geq f$ is valid on $L_{2}\left(\mathbf{R}^{n} \times \mathbf{R}^{m}\right)$ where $f$ is the form of the operator $F$ with

$$
F \sim L_{x_{2}}^{\alpha}\left(1+L_{x_{2}}\right)^{\alpha-\alpha^{\prime}}
$$

where

$$
\alpha=\left(1-\delta_{1}\right) /\left(1+\delta_{2}-\delta_{1}\right) \quad \text { and } \quad \alpha^{\prime}=\left(1-\delta_{1}^{\prime}\right) /\left(1+\delta_{2}^{\prime}-\delta_{1}^{\prime}\right)
$$

Proof First one has $h \geq a h_{\delta}$ and after a partial Fourier transformation, i.e., a transformation with respect to the $x_{2}$ variable, $H_{\delta}$ transforms to an operator $\widetilde{H}_{\delta}$ on $L_{2}\left(\mathbf{R}^{n}\right)$

$$
\widetilde{H}_{\delta}=H_{1}+c_{\delta_{2}, \delta_{2}^{\prime}}\left|p_{2}\right|^{2}
$$

where $H_{1}$ is the self-adjoint operator analyzed in Proposition [3.2. Therefore one may apply the latter proposition to $H_{1}$ to bound $\widetilde{H}_{\delta}$ below by a differential operator in the $\mathbf{R}^{n}$ variable. Now to proceed we again use the fractional Hardy inequality but to cover all the relevant cases we have to pass to a fractional power of $\widetilde{H}_{\delta}$. This is achieved with the aid of the following simple observation.

Lemma 3.6 Let $A$ and $B$ be positive self-adjoint operators such that the form sum $A+B$ is densely defined. Then the form sum $A^{1 / 2}+B^{1 / 2}$ is densely defined and

$$
(A+B)^{1 / 2} \geq 2^{-1 / 2}\left(A^{1 / 2}+B^{1 / 2}\right) .
$$

Similarly $A^{1 / 2^{n}}+B^{1 / 2^{n}}$ is densely defined and

$$
(A+B)^{1 / 2^{n}} \geq 2^{-1+2^{-n}}\left(A^{1 / 2^{n}}+B^{1 / 2^{n}}\right)
$$

for all positive integers $n$. 
Proof First one has $A+B \geq A$. Hence $(A+B)^{1 / 2} \geq A^{1 / 2}$ and $D\left(A^{1 / 2}\right) \supseteq D\left((A+B)^{1 / 2}\right)$. Similarly $(A+B)^{1 / 2} \geq B^{1 / 2}$ and $D\left(B^{1 / 2}\right) \supseteq D\left((A+B)^{1 / 2}\right)$. The first statement then follows because

$$
A+B=\left(A^{1 / 2}+B^{1 / 2}\right)^{2} / 2+\left(A^{1 / 2}-B^{1 / 2}\right)^{2} / 2 \geq\left(A^{1 / 2}+B^{1 / 2}\right)^{2} / 2 .
$$

The second statement follows by iteration.

The proof of Proposition 3.5 now continues by applying Lemma 3.6 to deduce that

$$
\widetilde{H}_{\delta}^{1 / 4} \geq a\left(H_{1}^{1 / 4}+c_{\delta_{2}, \delta_{2}^{\prime}}^{1 / 4}\left|p_{2}\right|^{1 / 2}\right)
$$

But if $\delta_{1} \leq \delta_{1}^{\prime}$ and $n \geq 2$, or $n=1$ and $\delta_{1}, \delta_{1}^{\prime} \in[0,1 / 2\rangle$, then, by Proposition 3.2.

$$
H_{1}^{1 / 4} \geq a L_{x_{1}}^{\left(1-\delta_{1}^{\prime}\right) / 4}\left(I+L_{x_{1}}^{\left(1-\delta_{1}^{\prime}\right) / 4}\right)^{-1+\sigma}
$$

where $\sigma=\left(1-\delta_{1}\right) /\left(1-\delta_{1}^{\prime}\right) \in\langle 0,1]$. But $0 \leq\left(1-\delta_{1}^{\prime}\right) / 4 \leq 1 / 4$. Therefore

$$
L_{x_{1}}^{\left(1-\delta_{1}^{\prime}\right) / 4} \geq a\left|x_{1}\right|^{-\left(1-\delta_{1}^{\prime}\right) / 2}
$$

by Lemma 3.3. Hence

$$
\begin{aligned}
L_{x_{1}}^{\left(1-\delta_{1}^{\prime}\right) / 4}\left(I+L_{x_{1}}^{\left(1-\delta_{1}^{\prime}\right) / 4}\right)^{-1+\sigma} & \geq a\left|x_{1}\right|^{-\left(1-\delta_{1}^{\prime}\right) / 2}\left(1+\left|x_{1}\right|^{-\left(1-\delta_{1}^{\prime}\right) / 2}\right)^{-1+\sigma} \\
& \geq a\left|x_{1}\right|^{-\left(\delta_{1}-\delta_{1}^{\prime}\right) / 2}\left(1+\left|x_{1}\right|\right)^{-\left(1-\delta_{1}\right) / 2}
\end{aligned}
$$

by Lemma 3.4. Then, however,

$$
\widetilde{H}_{\delta}^{1 / 4} \geq a\left(\left|x_{1}\right|^{-\left(\delta_{1}-\delta_{1}^{\prime}\right) / 2}\left(1+\left|x_{1}\right|\right)^{-\left(1-\delta_{1}\right) / 2}+c_{\delta_{2}, \delta_{2}^{\prime}}\left(x_{1}\right)^{1 / 4}\left|p_{2}\right|^{1 / 2}\right) .
$$

But the right hand side is a function of $\left|x_{1}\right|$ with a strictly positive minimum $m$ which is estimated by elementary arguments. The minimum value $m$ is a positive function of $\left|p_{2}\right|$ which then gives the bound $\widetilde{H}_{\delta} \geq M I$ with $M=m^{4}$.

In order to estimate the minimum $m$ we note that the first function in the last estimate, $x_{1} \mapsto\left|x_{1}\right|^{-\left(\delta_{1}-\delta_{1}^{\prime}\right) / 2}\left(1+\left|x_{1}\right|\right)^{-\left(1-\delta_{1}\right) / 2}$, is decreasing and the second function in the estimate, $x_{1} \mapsto c_{\delta_{2}, \delta_{2}^{\prime}}\left(x_{1}\right)^{1 / 4}\left|p_{2}\right|^{1 / 2}$, is increasing. Now if $\left|p_{2}\right|$ is small the graphs of the two functions intersect at a unique large value of $x_{1} \sim\left|p_{2}\right|^{-1 /\left(1+\delta_{2}^{\prime}-\delta_{1}^{\prime}\right)}$. At this point the value of the sum of the functions is proportional to $\left|p_{2}\right|^{\alpha^{\prime} / 2}$. Similarly if $\left|p_{2}\right|$ is large the graphs intersect at a small $x_{1} \sim\left|p_{2}\right|^{-1 /\left(1+\delta_{1}^{\prime}-\delta_{1}\right)}$ and the minimum value is proportional to $\left|p_{2}\right|^{\alpha / 2}$. Therefore

$$
m\left(p_{2}\right) \sim\left|p_{2}\right|^{\alpha^{\prime} / 2}\left(1+\left|p_{2}\right|^{2}\right)^{\left(\alpha-\alpha^{\prime}\right) / 4} \quad \text { and } \quad M\left(p_{2}\right) \sim\left|p_{2}\right|^{2 \alpha^{\prime}}\left(1+\left|p_{2}\right|^{2}\right)^{\alpha-\alpha^{\prime}}
$$

which is equivalent to the bound stated in the proposition.

If $n=1$ and $\delta_{1} \in[1 / 2,1\rangle$ or $\delta_{1}^{\prime} \in[1 / 2,1\rangle$ then the estimation procedure has to be slightly modified. Then Proposition 3.2 gives the lower bound

$$
H_{1}^{1 / 4} \geq a L_{x_{1}, N}^{\left(1-\delta_{1}^{\prime}\right) / 4}\left(I+L_{x_{1}, N}^{\left(1-\delta_{1}^{\prime}\right) / 4}\right)^{-1+\sigma}
$$

and $L_{x_{1}, N} \leq L_{x_{1}}$. Nevertheless if $\alpha<1 / 2$ then $L_{x_{1}, N}^{\alpha} \geq a L_{x_{1}}^{\alpha}$ since the boundary conditions do not affect small fractional powers. Therefore the bound (11) follows from (12) by another application of Lemma 3.4 . 
The case $\delta_{1} \geq \delta_{1}^{\prime}$ is similar but simpler.

Now we are prepared to estimate the crossnorm of the semigroup.

Proof of Proposition 3.1 It follows by definition that $h \sim h_{\delta}$ and then by combination of Propositions 3.2 and 3.5 that $h \geq f$ or $h \geq f_{N}$ where $f$ is the form of a multiplier $F\left(L_{x_{1}}, L_{x_{2}}\right)$ and $f_{N}$ the form of $F\left(L_{x_{1}, N}, L_{x_{2}}\right)$. Now consider the case $\delta_{1} \leq \delta_{1}^{\prime}$ and assume $n \geq 2$, or $n=1$ and $\delta_{1}, \delta_{1}^{\prime} \in[0,1 / 2\rangle$. Then one can apply Lemmas 2.4] and 2.5] with

$$
F\left(L_{x_{1}}, L_{x_{2}}\right)=a\left(L_{x_{1}}^{\left(1-\delta_{1}^{\prime}\right)}\left(1+L_{x_{1}}\right)^{-\left(\delta_{1}-\delta_{1}^{\prime}\right)}+L_{x_{2}}^{\alpha^{\prime}}\left(1+L_{x_{2}}\right)^{\alpha-\alpha^{\prime}}\right) .
$$

Therefore $V_{F}(r) \sim V_{F_{1}}(r) V_{F_{2}}(r)$ where

$$
V_{F_{1}}(r)=\left|\left\{p_{1}:\left|p_{1}\right|^{2\left(1-\delta_{1}^{\prime}\right)}\left(1+\left|p_{1}\right|^{2}\right)^{-\left(\delta_{1}-\delta_{1}^{\prime}\right)}<r^{2}\right\}\right|
$$

and

$$
V_{F_{2}}(r)=\left|\left\{p_{2}:\left|p_{2}\right|^{2 \alpha^{\prime}}\left(1+\left|p_{2}\right|^{2}\right)^{\alpha-\alpha^{\prime}}<r^{2}\right\}\right| .
$$

But $V_{F_{1}}(r) \sim r^{n\left(1 /\left(1-\delta_{1}^{\prime}\right), 1 /\left(1-\delta_{1}\right)\right)}$. Similarly $V_{F_{2}}(r) \sim r^{m\left(1 / \alpha^{\prime}, 1 / \alpha\right)}$. Therefore $V_{F}(r) \sim r^{\left(D^{\prime}, D\right)}$. Then the semigroup estimates of Proposition 3.1 follow from Lemma 2.5 .

The argument is similar if $\delta_{1} \geq \delta_{1}^{\prime}$ but one uses the second estimate of Proposition 3.2 . Finally if $n=1$ and $\delta_{1} \in[1 / 2,1\rangle$ or $\delta_{1}^{\prime} \in[1 / 2,1\rangle$ one can make an identical argument using the last statement of Proposition 3.2 and Lemma 2.6 which deals with the Neumann multipliers.

Remark 3.7 The arguments we have given for subellipticity estimates on $L_{2}\left(\mathbf{R}^{d} ; d x\right)$ also extend to weighted spaces such as $L_{2}\left(\mathbf{R}^{d} ;|x|^{\beta} d x\right)$. In this extension the Hardy inequality is replaced by the Caffarelli-Kohn-Nirenberg inequalities [CKN84.

\section{Comparison of kernels}

Proposition 3.1 gives bounds on the semigroup kernel associated with the Grušin operator which are uniform over $\mathbf{R}^{n} \times \mathbf{R}^{m}$. In this section we develop a method for transforming these bounds into bounds which better reflect the spatial behaviour, bounds expressed in terms of the corresponding Riemannian geometry. In particular we establish a comparison between the Grušin kernel and the kernel of a closely related non-degenerate operator. Our arguments are based on wave equation techniques and the initial problem is to establish that the wave equation has a finite speed of propagation when measured with respect to the Riemannian distance (44). We discuss this problem in the general context of the elliptic operators defined by the relaxation $h_{0}$ of the form $h$ corresponding to an elliptic operator $H$ given by (11).

First recall that the Riemannian distance is given by

$$
d(x ; y)=\sup _{\psi \in D}(\psi(x)-\psi(y))
$$

for all $x, y \in \mathbf{R}^{d}$ where

$$
D=\left\{\psi \in W^{1, \infty}\left(\mathbf{R}^{d}\right): \sum_{i, j=1}^{d} c_{i j}\left(\partial_{i} \psi\right)\left(\partial_{j} \psi\right) \leq 1\right\}
$$


Secondly, introduce the corresponding set-theoretic distance by

$$
d(A ; B)=\inf _{x \in A, y \in B} d(x ; y)
$$

where $A$ and $B$ are general measurable sets.

Our first aim is to establish the following basic estimates.

Proposition 4.1 Let $S^{(0)}$ denote the semigroup generated by the self adjoint operator $H_{0}$ associated with the relaxation $h_{0}$ of the elliptic form $h$ with coefficients $C=\left(c_{i j}\right)$. Then for each pair of open subsets $A, B$ of $\mathbf{R}^{d}$

$$
\left|\left(\varphi_{A}, S_{t}^{(0)} \varphi_{B}\right)\right| \leq e^{-d(A ; B)^{2}(4 t)^{-1}}\left\|\varphi_{A}\right\|_{2}\left\|\varphi_{B}\right\|_{2}
$$

for all $\varphi_{A} \in L_{2}(A), \varphi_{B} \in L_{2}(B)$ and $t>0$ with the convention $e^{-\infty}=0$. Moreover, the corresponding wave equation has a finite speed of propagation in the sense that

$$
\left(\varphi_{A}, \cos \left(t H_{0}^{1 / 2}\right) \varphi_{B}\right)=0
$$

for all $\varphi_{A} \in L_{2}(A), \varphi_{B} \in L_{2}(B)$ and all $t$ with $|t| \leq d(A ; B)$.

Proof First let $\psi \in D$ and introduce the one-parameter family of multiplication operators $\rho \rightarrow U_{\rho}=e^{\rho \psi}$ on $L_{2}\left(\mathbf{R}^{d}\right)$. Then $\left\|U_{\rho} S_{t}^{(0)} U_{\rho}^{-1}\right\|_{2 \rightarrow 2} \leq e^{\rho^{2} t}$. This is a standard estimate for strongly elliptic operators which extends to general elliptic operators (see, for example, the proof of Proposition 3.1 in [ERSZ06]). Now

$$
\begin{aligned}
\left|\left(\varphi_{A}, S_{t}^{(0)} \varphi_{B}\right)\right| & =\left|\left(U_{\rho}^{-1} \varphi_{A},\left(U_{\rho} S_{t}^{(0)} U_{\rho}^{-1}\right) U_{\rho} \varphi_{B}\right)\right| \\
& \leq e^{\rho^{2} t}\left\|U_{\rho}^{-1} \varphi_{A}\right\|_{2}\left\|U_{\rho} \varphi_{B}\right\|_{2} \leq e^{-\rho d_{\psi}(A ; B)} e^{\rho^{2} t}\left\|\varphi_{A}\right\|_{2}\left\|\varphi_{B}\right\|_{2}
\end{aligned}
$$

where

$$
d_{\psi}(A ; B)=\inf _{x \in A, y \in B}(\psi(x)-\psi(y)) .
$$

Therefore, optimizing over $\psi$ and $\rho$ one has

$$
\left|\left(\varphi_{A}, S_{t}^{(0)} \varphi_{B}\right)\right| \leq e^{-\hat{d}(A ; B)^{2}(4 t)^{-1}}\left\|\varphi_{A}\right\|_{2}\left\|\varphi_{B}\right\|_{2}
$$

where

$$
\hat{d}(A ; B)=\sup _{\psi \in D} d_{\psi}(A ; B) .
$$

These estimates are valid for all measurable $A, B$ and all $\varphi_{A} \in L_{2}(A), \varphi_{B} \in L_{2}(B)$.

Since $d_{\psi}(A ; B) \leq \psi(x)-\psi(y)$ for all $x \in A$ and $y \in B$ it follows that $\hat{d}(A ; B) \leq d(A ; B)$ again for all measurable $A$ and $B$. But the latter inequality has a partial converse.

Lemma 4.2 If $A$ and $B$ are compact subsets then $\hat{d}(A ; B)=d(A ; B)$.

Remarks 4.3 1. The proof is an interplay between compactness and continuity. It uses very little structure of the set $D$. Indeed it suffices for the proof that $\varphi \in D$ and $c \in \mathbf{R}$ imply $-\varphi+c \in D$ and $\varphi_{1}, \varphi_{2} \in D$ imply $\varphi_{1} \vee \varphi_{2}, \varphi_{1} \wedge \varphi_{2} \in D$. These properties are easily verified.

2. It is not necessarily the case that $\left\{\varphi_{n}\right\}_{n \geq 1} \in D$ implies $\sup _{n \geq 1} \varphi_{n} \in D$ or $\inf _{n \geq 1} \varphi_{n} \in D$. Proposition 6.5 in [ERSZ06], and its proof, give an example of a decreasing sequence $\chi_{n}$ of functions in $D$ such that $\inf _{n} \chi_{n} \notin W^{1, \infty}$. 
Proof of Lemma 4.2 Since $\hat{d}(A ; B) \leq d(A ; B)$ for all measurable $A$ and $B$ it suffices to prove $\hat{d}(A ; B) \geq d(A ; B)$ for $A$ and $B$ compact.

Fix $x \in A$ and $y \in B$. Then for each $\varepsilon>0$ there is a $\psi_{x, y} \in D$ such that $\psi_{x, y}(x)=0$ and $\psi_{x, y}(y) \geq d(x ; y)-\varepsilon / 4$. Next since $\psi_{x, y}$ is continuous there exists an open neighbourhood $U_{y}$ of $y$ such that

$$
\psi_{x, y}(z) \geq d(x ; y)-\varepsilon / 2 \geq d(A ; B)-\varepsilon / 2
$$

for and $z \in U_{y}$. Then $B \subset \bigcup_{y \in B} U_{y}$ and since $B$ is compact there exist $y_{1}, \ldots, y_{n} \in B$ such that $B \subset \bigcup_{k=1}^{n} U_{y_{k}}$. Set $\psi_{x}=\sup _{1 \leq k \leq n} \psi_{x, y_{k}}$ then $\psi_{x} \in D, \psi_{x}(x)=0$ and

$$
\inf _{z \in B} \psi_{x}(z) \geq \min _{1 \leq k \leq n} d\left(x ; y_{k}\right)-\varepsilon / 2 \geq d(A ; B)-\varepsilon / 2
$$

But since $\psi_{x}$ is continuous there is an open neighbourhood $U_{x}$ of $x$ such that $\psi_{x}(z) \leq \varepsilon / 2$ for all $z \in U_{x}$. Then by repeating the above covering argument one can select $x_{1}, \ldots, x_{m} \in A$ such that $\psi=\inf _{1 \leq k \leq m} \psi_{x_{k}}$ satisfies $\psi(z) \leq \varepsilon / 2$ for all $z \in A$. In addition one still has $\psi(z) \geq d(A ; B)-\varepsilon / 2$ for all $z \in B$. Therefore

$$
\inf _{x \in A, y \in B}(\psi(y)-\psi(x)) \geq d(A ; B)-\varepsilon .
$$

Thus $\hat{d}(A ; B) \geq d(A ; B)-\varepsilon$. Since $\varepsilon>0$ is arbitrary one deduces that $\hat{d}(A ; B) \geq d(A ; B)$ for $A$ and $B$ compact.

Now we can complete the proof of Proposition 4.1

End of proof of Proposition 4.1 Let $\varphi_{1} \in L_{2}(A)$ have compact support $U_{1} \subset A$ and $\varphi_{2} \in L_{2}(B)$ have compact support $U_{2} \subset B$. Then it follows from (17) and Lemma 4.2 that

$$
\begin{aligned}
\left|\left(\varphi_{1}, S_{t}^{(0)} \varphi_{2}\right)\right| & \leq e^{-\hat{d}\left(U_{1} ; U_{2}\right)^{2}(4 t)^{-1}}\left\|\varphi_{1}\right\|_{2}\left\|\varphi_{2}\right\|_{2} \\
& =e^{-d\left(U_{1} ; U_{2}\right)^{2}(4 t)^{-1}}\left\|\varphi_{1}\right\|_{2}\left\|\varphi_{2}\right\|_{2} \leq e^{-d(A ; B)^{2}(4 t)^{-1}}\left\|\varphi_{1}\right\|_{2}\left\|\varphi_{2}\right\|_{2} .
\end{aligned}
$$

But if $A$ is open then the functions of compact support in $L_{2}(A)$ are dense and similarly for $B$. Therefore the first statement (15) of the proposition follows by continuity. The second statement (16) is a direct consequence (see, for example, [ERSZ06] Lemma 3.3).

An equivalent way of expressing the finite speed of propagation is the following.

Lemma 4.4 Let $A$ be an open subset and $F$ a closed subset with $A \subset F$. Then

$$
\cos \left(t H_{0}^{1 / 2}\right) L_{2}(A) \subseteq L_{2}(F)
$$

for all $t \in \mathbf{R}$ with $|t| \leq d\left(A ; F^{\mathrm{c}}\right)$.

Proof Let $B$ be an open subset of $F^{\mathrm{c}}$. Then $d\left(A ; F^{\mathrm{c}}\right) \leq d(A ; B)$. Therefore $L_{2}(B) \perp$ $\cos \left(t H_{0}^{1 / 2}\right) L_{2}(A)$ for all $t \in \mathbf{R}$ with $|t| \leq d\left(A ; F^{\mathrm{c}}\right)$ by (16). Hence $L_{2}\left(F^{\mathrm{c}}\right) \perp \cos \left(t H_{0}^{1 / 2}\right) L_{2}(A)$ and one must have $\cos \left(t H_{0}^{1 / 2}\right) L_{2}(A) \subseteq L_{2}(F)$.

The subsequent comparison theorem depends on a generalization of the propagation property which emphasizes the local nature. As a preliminary let $h_{1}$ and $h_{2}$ be two elliptic forms with coefficients $C_{1}=\left(c_{i j}^{(1)}\right)$ and $C_{2}=\left(c_{i j}^{(2)}\right)$ and let $H_{1,0}$ and $H_{2,0}$ denote the corresponding relaxations. Moreover, assume that $C_{1} \geq C_{2}$ and $C_{1} \geq \mu I>0$. In particular $D\left(h_{1}\right) \subseteq D\left(h_{2}\right) \subseteq W^{1,2}\left(\mathbf{R}^{d}\right)$ and $d_{1}(x ; y) \leq d_{2}(x ; y)$ for all $x, y$ where $d_{1}$ and $d_{2}$ denote the Riemannian distances associated with $C_{1}$ and $C_{2}$. Let $U=\operatorname{supp}\left(C_{1}-C_{2}\right)$ and set $F=\overline{U^{\mathrm{c}}}$. 
Lemma 4.5 If $A$ is an open subset of the closed subset $F$ then

$$
\cos \left(t H_{1,0}^{1 / 2}\right) \varphi_{A}=\cos \left(t H_{2,0}^{1 / 2}\right) \varphi_{A}
$$

for all $\varphi_{A} \in L_{2}(A)$ and all $t \in \mathbf{R}$ with $|t| \leq d_{1}(A ; U)$.

Proof If $C_{1}$ and $C_{2}$ are strongly elliptic this result follows from the proof of Proposition 3.15 in [ERS06. The extension to the more general situation can then be made by approximation as follows.

Let $N>\varepsilon>0$. Set $C_{1, N, \varepsilon}=\left(C_{1} \wedge N I\right)+\varepsilon I$ and $C_{2, N, \varepsilon}=\left(C_{2} \wedge N I\right)+\varepsilon I$. Then the lemma is valid for the corresponding strongly elliptic operators $H_{1, N, \varepsilon}, H_{2, N, \varepsilon}$. But as $N \rightarrow \infty$ these operators converge in the strong resolvent sense to $H_{1, \varepsilon}=H_{1}+\varepsilon I$ and $H_{2, \varepsilon}=H_{2}+\varepsilon I$, respectively. Since $H_{1, \varepsilon} \geq H_{1, N, \varepsilon} \geq H_{2, N, \varepsilon}$ it follows that

$$
\cos \left(t H_{1, \varepsilon}^{1 / 2}\right) \varphi_{A}=\cos \left(t H_{2, \varepsilon}^{1 / 2}\right) \varphi_{A}
$$

for all $t \in \mathbf{R}$ with $|t| \leq d_{1, \varepsilon}(A ; U)$ where $d_{1, \varepsilon}$ denotes the Riemannian distance associated with $C_{1, \varepsilon}$.

Finally it follows that $H_{1, \varepsilon}$ and $H_{2, \varepsilon}$ converge in the strong resolvent sense to $H_{1,0}$ and $H_{2,0}$, respectively, as $\varepsilon \rightarrow 0$. Moreover, since $C_{1} \leq C_{1, \varepsilon} \leq\left(1+\varepsilon \mu^{-1}\right) C_{1}$ it follows that $\left(1+\varepsilon \mu^{-1}\right)^{-1 / 2} d_{1}(A ; B) \leq d_{1, \varepsilon}(A ; B) \leq d_{1}(A ; B)$ for all measurable $A$, $B$. Hence $d_{1, \varepsilon}(A ; B) \rightarrow d_{1}(A ; B)$ as $\varepsilon \rightarrow 0$. Therefore the statement of the lemma follows in the limit.

Now we are prepared to establish the principal comparison result. In the sequel $S^{(1,0)}$, $S^{(2,0)}$ denote the semigroups generated by $H_{1,0}, H_{2,0}$ and $K^{(1,0)}, K^{(2,0)}$ denote the corresponding kernels.

Theorem 4.6 Adopt the foregoing notation and assumptions. Let $\chi_{A}$ denote the characteristic function of the open subset $A$ of F. Set

$$
M_{N}(t)=\left\|\chi_{A}\left(I+t^{2} H_{1,0}\right)^{-N} \chi_{A}\right\|_{1 \rightarrow \infty}+\left\|\chi_{A}\left(I+t^{2} H_{2,0}\right)^{-N} \chi_{A}\right\|_{1 \rightarrow \infty}
$$

for $N \in \mathbf{N}$.

Then there is an $a_{N}>0$ such that

$$
\sup _{x, y \in A}\left|K_{t}^{(1,0)}(x ; y)-K_{t}^{(2,0)}(x ; y)\right| \leq a_{N} M_{N}(t / \rho)\left(\rho^{2} / t\right)^{-1 / 2} e^{-\rho^{2} /(4 t)}
$$

for all $t>0$ where $\rho=d_{1}(A ; U)$.

Note that there is no reason that $M_{N}$ is finite. Subsequently we give conditions which ensure that $M_{N}$ is indeed finite for large $N$.

The proof of the theorem is based on the estimates developed in [Sik96] and [Sik04]. As a preliminary we need some properties of functions of the operators $H_{0,1}$ and $H_{2,0}$.

First, let $\Psi$ be an even bounded Borel function with Fourier transform $\widetilde{\Psi}$ satisfying supp $\widetilde{\Psi} \subseteq[-\rho, \rho]$ where $\rho>0$. Then for each pair of open subsets $B$ and $C$

$$
\left(\varphi_{B}, \Psi\left(H_{1,0}^{1 / 2}\right) \varphi_{C}\right)=0=\left(\varphi_{B}, \Psi\left(H_{2,0}^{1 / 2}\right) \varphi_{C}\right)
$$


for all $\varphi_{B} \in L_{2}(B), \varphi_{C} \in L_{2}(C)$ where $\rho \leq d_{1}(B ; C)$. This follows for $H_{1,0}$ from the representation

$$
\Psi\left(H_{1,0}^{1 / 2}\right)=(2 \pi)^{-1 / 2} \int_{\mathbf{R}} d t \widetilde{\Psi}(t) \exp \left(i t H_{1,0}^{1 / 2}\right)=(2 \pi)^{-1 / 2} \int_{-\rho}^{\rho} d t \widetilde{\Psi}(t) \cos \left(t H_{1,0}^{1 / 2}\right)
$$

and condition (16). The argument for $H_{2,0}$ is similar.

Secondly, we have the key lemma.

Lemma 4.7 Let $\Psi$ be an even bounded Borel function with Fourier transform $\widetilde{\Psi}$ satisfying $\operatorname{supp} \widetilde{\Psi} \subseteq[-2 \rho, 2 \rho]$. Then

$$
\chi_{A} \Psi\left(H_{1,0}^{1 / 2}\right) \chi_{A}=\chi_{A} \Psi\left(H_{2,0}^{1 / 2}\right) \chi_{A}
$$

Proof Since $A \subset F$ and $\rho=d_{1}(A ; U)$ one has

$$
\cos \left(t H_{1,0}^{1 / 2}\right) \varphi_{A}=\cos \left(t H_{2,0}^{1 / 2}\right) \varphi_{A}
$$

for all $\varphi_{A} \in L_{2}(A)$ and $t \in \mathbf{R}$ with $|t| \leq \rho$ by Lemma 4.5.

Next remark that

$$
\begin{aligned}
& \left(\psi_{A}, \cos \left(2 t H_{1,0}^{1 / 2}\right) \varphi_{A}\right)-\left(\psi_{A}, \cos \left(2 t H_{2,0}^{1 / 2}\right) \varphi_{A}\right) \\
& \quad=2\left(\left(\cos \left(t H_{1,0}^{1 / 2}\right) \psi_{A}, \cos \left(t H_{1,0}^{1 / 2}\right) \varphi_{A}\right)-\left(\cos \left(t H_{2,0}^{1 / 2}\right) \psi_{A}, \cos \left(t H_{2,0}^{1 / 2}\right) \varphi_{A}\right)\right)=0
\end{aligned}
$$

for $|t| \leq \rho$ and $\psi_{A}, \varphi_{A} \in L_{2}(A)$. Then, however, one has

$$
\begin{aligned}
\left(\psi, \chi_{A} \Psi\left(H_{1,0}^{1 / 2}\right) \chi_{A} \varphi\right) & =(2 \pi)^{-1 / 2} \int_{-2 \rho}^{2 \rho} d t \widetilde{\Psi}(t)\left(\chi_{A} \psi, \cos \left(t H_{1,0}^{1 / 2}\right) \chi_{A} \varphi\right) \\
& =(2 \pi)^{-1 / 2} \int_{-2 \rho}^{2 \rho} d t \widetilde{\Psi}(t)\left(\chi_{A} \psi, \cos \left(t H_{2,0}^{1 / 2}\right) \chi_{A} \varphi\right)=\left(\psi, \chi_{A} \Psi\left(H_{2,0}^{1 / 2}\right) \chi_{A} \varphi\right)
\end{aligned}
$$

for all $\varphi \in L_{2}\left(\mathbf{R}^{d}\right)$.

Now we are prepared to prove the theorem. We use the notation $K_{T}$ for the kernel of an operator $T$.

Proof of Theorem 4.6 Let $\psi \in C^{\infty}(\mathbf{R})$ be an increasing function with

$$
\psi(u)= \begin{cases}0 & \text { if } u \leq-1 \\ 1 & \text { if } u \geq-1 / 2\end{cases}
$$

Then for $s>1$ define the family of functions $\varphi_{s}$ such that

$$
\varphi_{s}(u)=\psi(s(|u|-s))
$$

Next define functions $\widetilde{\Phi}_{s}$ and $\widetilde{\Psi}_{s}$ by

$$
\widetilde{\Phi}_{s}(u)=(4 \pi)^{-1 / 2} \exp \left(-u^{2} / 4\right)-\widetilde{\Psi}_{s}(u)=\varphi_{s}(u)(4 \pi)^{-1 / 2} \exp \left(-u^{2} / 4\right)
$$


Then the inverse Fourier transforms satisfy $\Phi_{s}(\lambda)+\Psi_{s}(\lambda)=(2 \pi)^{-1 / 2} \exp \left(-\lambda^{2}\right)$ and

$$
S_{t}^{(i, 0)}=\exp \left(-t H_{i, 0}\right)=\Phi_{s}\left(\left(t H_{i, 0}\right)^{1 / 2}\right)+\Psi_{s}\left(\left(t H_{i, 0}\right)^{1 / 2}\right)
$$

for $i=1,2$. Integration by parts $2 N$ times yields

$$
\int d u e^{-u^{2} / 4} e^{-i u \lambda} \varphi_{s}(u)=\int d u e^{-u^{2} / 4-i \lambda u} \underbrace{\left(\frac{1}{u / 2+i \lambda}\left(\cdots\left(\frac{1}{u / 2+i \lambda} \varphi_{s}(u)\right)^{\prime} \cdots\right)^{\prime}\right)^{\prime}}_{2 N} .
$$

Hence for any $N \in \mathbf{N}$ and $s>1$ there is an $a_{N}>0$ such that

$$
\left|\Phi_{s}(\lambda)\right| \leq a_{N} \frac{1}{s\left(1+\lambda^{2} / s^{2}\right)^{N}} e^{-s^{2} / 4}
$$

with the value of $a_{N}$ depending only on $N$.

Next note that supp $\widetilde{\Psi}_{s} \subseteq\left[-s+(2 s)^{-1}, s-(2 s)^{-1}\right] \subset[-s, s]$. So setting $s_{\rho}=2 \rho t^{-1 / 2}$ one has

$$
\chi_{A} \Psi_{s_{\rho}}\left(\left(t H_{1,0}\right)^{1 / 2}\right) \chi_{A}=\chi_{A} \Psi_{s_{\rho}}\left(\left(t H_{2,0}\right)^{1 / 2}\right) \chi_{A}
$$

by Lemma 4.7 and rescaling with $t^{1 / 2}$. Hence one deduces from (24) that

$$
\left.\left.\chi_{A}\left(S_{t}^{(1,0)}-S_{t}^{(2,0)}\right) \chi_{A}=\chi_{A} \Phi_{s_{\rho}}\left(\left(t H_{1,0}\right)^{1 / 2}\right)\right) \chi_{A}-\chi_{A} \Phi_{s_{\rho}}\left(\left(t H_{2,0}\right)^{1 / 2}\right)\right) \chi_{A} .
$$

Therefore

$$
\begin{aligned}
\sup _{x, y \in A} \mid K_{t}^{(1,0)}(x ; y)- & K_{t}^{(2,0)}(x ; y) \mid \\
& \leq\left\|\chi_{A} \Phi_{s_{\rho}}\left(\left(t H_{1,0}\right)^{1 / 2}\right) \chi_{A}\right\|_{1 \rightarrow \infty}+\left\|\chi_{A} \Phi_{s_{\rho}}\left(\left(t H_{2,0}\right)^{1 / 2}\right) \chi_{A}\right\|_{1 \rightarrow \infty} .
\end{aligned}
$$

Now let $\Theta_{s_{\rho}}$ be a possibly complex function such that $\Theta_{s_{\rho}}(\lambda)^{2}=\Phi_{s_{\rho}}\left(t^{1 / 2} \lambda\right)$. Then

$$
\begin{aligned}
& \left.\| \chi_{A} \Phi_{s_{\rho}}\left(\left(t H_{i, 0}\right)^{1 / 2}\right)\right) \chi_{A} \|_{1 \rightarrow \infty}=\left(\left\|\chi_{A} \Theta_{s_{\rho}}\left(\left(H_{i, 0}\right)^{1 / 2}\right)\right\|_{2 \rightarrow \infty}\right)^{2} \\
& \quad \leq\left(\left\|\left(I+t^{2} H_{i, 0} / \rho^{2}\right)^{N / 2} \Theta_{s_{\rho}}\left(\left(H_{i, 0}\right)^{1 / 2}\right)\right\|_{2 \rightarrow 2}\right)^{2}\left(\left\|\chi_{A}\left(I+t^{2} H_{i, 0} / \rho^{2}\right)^{-N / 2}\right\|_{2 \rightarrow \infty}\right)^{2}
\end{aligned}
$$

But

$$
\begin{aligned}
\left|\Theta_{s_{\rho}}(\lambda)\left(1+t^{2} \lambda^{2} / \rho^{2}\right)^{N / 2}\right|^{2} & =\left|\Phi_{s_{\rho}}\left(t^{1 / 2} \lambda\right)\left(1+t\left(t^{1 / 2} \lambda\right)^{2} / \rho^{2}\right)^{N}\right| \\
& =\left|\Phi_{s_{\rho}}\left(t^{1 / 2} \lambda\right)\left(1+4\left(t^{1 / 2} \lambda\right)^{2} / s_{\rho}^{2}\right)^{N}\right|
\end{aligned}
$$

Therefore

$$
\begin{aligned}
\left(\left\|\left(I+t^{2} H_{i, 0} / \rho^{2}\right)^{N / 2} \Theta_{s_{\rho}}\left(\left(H_{i, 0}\right)^{1 / 2}\right)\right\|_{2 \rightarrow 2}\right)^{2} & =\sup _{\lambda \geq 0}\left|\Theta_{s_{\rho}}(\lambda)\left(1+t^{2} \lambda^{2} / \rho^{2}\right)^{N / 2}\right|^{2} \\
& =\sup _{\lambda \geq 0}\left|\Phi_{s_{\rho}}\left(t^{1 / 2} \lambda\right)\left(1+4\left(t^{1 / 2} \lambda\right)^{2} / s_{\rho}^{2}\right)^{N}\right| \\
& \leq a_{N}\left(s_{\rho} / 2\right)^{-1} e^{-s_{\rho}^{2} / 16}=a_{N}\left(\rho^{2} / t\right)^{-1 / 2} e^{-\rho^{2} /(4 t)} .
\end{aligned}
$$


Combining these estimates gives

$$
\sup _{x, y \in A}\left|K_{t}^{(1,0)}(x ; y)-K_{t}^{(2,0)}(x ; y)\right|=a_{N} M_{N}(t / \rho)\left(\rho^{2} / t\right)^{-1 / 2} e^{-\rho^{2} /(4 t)}
$$

which establishes the statement of the theorem.

The statement of the theorem can be reformulated in terms of a priori bounds on the semigroups $S^{(1,0)}$ and $S^{(2,0)}$. But this requires a uniform estimate on the crossnorms $\left\|S^{(i, 0)}\right\|_{1 \rightarrow \infty}$.

If $h$ is strongly elliptic then $\left\|S_{t}^{(0)}\right\|_{1 \rightarrow \infty} \leq a t^{-d / 2}$ for all $t>0$ and we will assume analogous bounds

$$
\left\|S_{t}^{(0)}\right\|_{1 \rightarrow \infty} \leq a V(t)^{-1}
$$

where $V$ is a positive increasing function which satisfies the doubling property

$$
V(2 t) \leq a V(t)
$$

for some $a>0$ and all $t>0$. It follows from (27) that there are $a, \widetilde{D}>0$ such that

$$
V(s) \leq a(s / t)^{\widetilde{D}} V(t)
$$

for all $s \geq t>0$. The parameter $\widetilde{D}$ is the doubling dimension, although it need not be an integer.

Corollary 4.8 Adopt the hypotheses and notation of Theorem 4.6. Let $V$ be a positive increasing function which satisfies the doubling property (27). Assume that

$$
\left\|\chi_{A} S_{t}^{(1,0)} \chi_{A}\right\|_{1 \rightarrow \infty} \vee\left\|\chi_{A} S_{t}^{(2,0)} \chi_{A}\right\|_{1 \rightarrow \infty} \leq V(t)^{-1}
$$

for all $t>0$.

Then there is an $a>0$ such that

$$
\sup _{x, y \in A}\left|K_{t}^{(1,0)}(x ; y)-K_{t}^{(2,0)}(x ; y)\right| \leq a V\left(t^{2} / \rho^{2}\right)^{-1}\left(\rho^{2} / t\right)^{-1 / 2} e^{-\rho^{2} /(4 t)}
$$

for all $t>0$, where $\rho=d_{1}(A ; U)$.

Proof It follows by Laplace transformation that

$$
\begin{aligned}
\left\|\chi_{A}\left(I+t^{2} H_{0}\right)^{-N} \chi_{A}\right\|_{1 \rightarrow \infty} & \leq \frac{1}{(N-1) !} \int_{0}^{\infty} d s s^{N-1} e^{-s}\left\|\chi_{A} S_{s t^{2}}^{(0)} \chi_{A}\right\|_{1 \rightarrow \infty} \\
& \leq \frac{1}{(N-1) !} \int_{0}^{\infty} d s s^{N-1} e^{-s} V\left(s t^{2}\right)^{-1} \leq a_{N} V\left(t^{2}\right)^{-1}
\end{aligned}
$$

for all $t>0$ with $a_{N}$ finite if $N>\widetilde{D}$. The last step uses the doubling property (28) in the form $V\left(t^{2}\right) \leq \rho s^{-\widetilde{D}} V\left(s t^{2}\right)$ for $s \leq 1$. Therefore $M_{N}(t) \leq 2 a_{N} V\left(t^{2}\right)^{-1}$ and the statement of the corollary is an immediate consequence of Theorem 4.6.

In Section [6] we apply Corollary 4.8 to the Grušin operator $H$. Then we set $H_{2}=H$ and $H_{1}$ a Grušin operator with no local degeneracies but with the same growth properties for $\left|x_{1}\right| \geq 1$. 


\section{Volume estimates}

In this section we return to the analysis of the general Grušin operator $H$. Our aim is to calculate the Riemannian distance $d(\cdot ; \cdot)$, given by (4), and the volume of the corresponding balls $B(x ; r)=\left\{y \in \mathbf{R}^{n} \times \mathbf{R}^{m}: d(x ; y)<r\right\}$, up to equivalence. In Section 3 we established uniform bounds on the crossnorm $\left\|S_{t}\right\|_{1 \rightarrow \infty}$ on the semigroup generated by the relaxation of $H$ and these automatically give bounds on the semigroup kernel $K_{t}$ which are uniform over $\mathbf{R}^{n} \times \mathbf{R}^{m}$. But in Section [ we will improve these uniform bounds, with the help of the comparison results of Section 4 to obtain bounds $K_{t}(x ; y) \leq a\left(\left|B\left(x ; t^{1 / 2}\right)\right|\left|B\left(y ; t^{1 / 2}\right)\right|\right)^{-1 / 2}$ in terms of the volume of the balls.

First, remark that if $C_{1}, C_{2}$ are two positive symmetric matrices whose entries are measurable functions and $d_{1}(\cdot ; \cdot), d_{2}(\cdot ; \cdot)$ the corresponding distances then $C_{1} \sim C_{2}$ implies $d_{1}(\cdot ; \cdot) \sim d_{2}(\cdot ; \cdot)$. In particular if $a C_{1} \leq C_{2} \leq b C_{1}$ then $b^{-1 / 2} d_{1}(x ; y) \leq d_{2}(x ; y) \leq$ $a^{-1 / 2} d_{1}(x ; y)$ for all $x, y \in \mathbf{R}^{d}$. Moreover, the corresponding balls $B_{1}, B_{2}$ satisfy

$$
B_{2}\left(x ; b^{-1 / 2} r\right) \subseteq B_{1}(x ; r) \subseteq B_{2}\left(x ; a^{-1 / 2} r\right)
$$

for all $x \in \mathbf{R}^{d}$ and all $r>0$. Then $\left|B_{1}\right| \sim\left|B_{2}\right|$ and if $\left|B_{1}\right|$ satisfies the doubling property with doubling dimension $\widetilde{D}$ then $\left|B_{2}\right|$ also satisfies the property with the same dimension, and conversely.

Secondly, in considering the Grušin operator the coefficient matrix $C \sim C_{\delta}$ and to calculate the Riemannian distance, up to equivalence, we may make a convenient choice of the $C_{\delta}$. In particular we may choose $C_{\delta}$ such that its entries $c_{\delta_{1}, \delta_{1}^{\prime}}, c_{\delta_{2}, \delta_{2}^{\prime}}$ are continuous functions over $\mathbf{R}^{n}$. In fact we may assume $c_{\delta_{1}, \delta_{1}^{\prime}} \in C^{\delta_{1}}\left(\mathbf{R}^{n}\right)$ and $c_{\delta_{2}, \delta_{2}^{\prime}} \in C^{\delta_{2}}\left(\mathbf{R}^{n}\right)$. The continuity of the coefficients then allow us to appeal to path arguments in the computation of the Riemannian distance. Specifically the Riemannian distance defined by (4) is equivalent to the shortest distance of paths measured with respect to a continuous choice of $C_{\delta}$.

Thirdly, recall that we always assume that $0 \leq \delta_{1}, \delta_{1}^{\prime}<1$ and $0 \leq \delta_{2}, \delta_{2}^{\prime}$. Next let $B\left(x_{1}, x_{2} ; r\right)$ denote the ball with centre $x=\left(x_{1}, x_{2}\right)$ and $\left|B\left(x_{1}, x_{2} ; r\right)\right|$ its volume. Further let $D=\left(n+m\left(1+\delta_{2}-\delta_{1}\right)\right)\left(1-\delta_{1}\right)^{-1}$ and $D^{\prime}=\left(n+m\left(1+\delta_{2}^{\prime}-\delta_{1}^{\prime}\right)\right)\left(1-\delta_{1}^{\prime}\right)^{-1}$ denote the parameters occurring in the uniform bounds of Proposition 3.1. Next define the function $\Delta_{\delta}$ by the formula

$$
\Delta_{\delta}\left(x_{1}, x_{2} ; y_{1}, y_{2}\right)= \begin{cases}\left|x_{2}-y_{2}\right| /\left(\left|x_{1}\right|+\left|y_{1}\right|\right)^{\left(\delta_{2}, \delta_{2}^{\prime}\right)} & \text { if }\left|x_{2}-y_{2}\right| \leq\left(\left|x_{1}\right|+\left|y_{1}\right|\right)^{\left(\rho, \rho^{\prime}\right)} \\ \left|x_{2}-y_{2}\right|^{\left(1-\gamma, 1-\gamma^{\prime}\right)} & \text { if }\left|x_{2}-y_{2}\right| \geq\left(\left|x_{1}\right|+\left|y_{1}\right|\right)^{\left(\rho, \rho^{\prime}\right)}\end{cases}
$$

where $\rho=1+\delta_{2}-\delta_{1}, \rho^{\prime}=1+\delta_{2}^{\prime}-\delta_{1}^{\prime}, \gamma=\delta_{2} / \rho$ and $\gamma^{\prime}=\delta_{2}^{\prime} / \rho^{\prime}$. Now set

$$
D_{\delta}\left(x_{1}, x_{2} ; y_{1}, y_{2}\right)=\left|x_{1}-y_{1}\right| /\left(\left|x_{1}\right|+\left|y_{1}\right|\right)^{\left(\delta_{1}, \delta_{1}^{\prime}\right)}+\Delta_{\delta}\left(x_{1}, x_{2} ; y_{1}, y_{2}\right) \text {. }
$$

Note that

$$
\Delta_{\delta}\left(x_{1}, x_{2} ; y_{1}, y_{2}\right) \sim \frac{\left|x_{2}-y_{2}\right|}{\left(\left|x_{1}\right|+\left|y_{1}\right|\right)^{\left(\delta_{2}, \delta_{2}^{\prime}\right)}+\left|x_{2}-y_{2}\right|^{\left(\gamma, \gamma^{\prime}\right)}} .
$$

Note also that if $\left(\left|x_{1}\right|+\left|y_{1}\right|\right)^{\left(\rho, \rho^{\prime}\right)}=\left|x_{2}-y_{2}\right|$ then

$$
\frac{\left|x_{2}-y_{2}\right|}{\left(\left|x_{1}\right|+\left|y_{1}\right|\right)^{\left(\delta_{2}, \delta_{2}^{\prime}\right)}}=\frac{\left|x_{2}-y_{2}\right|}{\left|x_{2}-y_{2}\right|^{\left(\gamma, \gamma^{\prime}\right)}}=\left|x_{2}-y_{2}\right|^{\left(1-\gamma, 1-\gamma^{\prime}\right)}
$$

so $\Delta_{\delta}$ is a continuous function of the variables $x_{1}, x_{2}, y_{1}, y_{2}$. The main result in this section is the following. 
Proposition 5.1 Consider the general Grušin operator with coefficients $C \sim C_{\delta}$. If $d_{\delta}$ is the Riemannian distance defined by (4) with the coefficients $C_{\delta}$ then

$$
d_{\delta}\left(x_{1}, x_{2} ; y_{1}, y_{2}\right) \sim D_{\delta}\left(x_{1}, x_{2} ; y_{1}, y_{2}\right)
$$

Moreover, the volume of the corresponding balls satisfy

$$
\left|B\left(x_{1}, x_{2} ; r\right)\right| \sim \begin{cases}r^{\left(D, D^{\prime}\right)} & \text { if } r \geq\left|x_{1}\right|^{\left(1-\delta_{1}, 1-\delta_{1}^{\prime}\right)} \\ r^{n+m}\left|x_{1}\right|^{\left(\beta, \beta^{\prime}\right)} & \text { if } r \leq\left|x_{1}\right|^{\left(1-\delta_{1}, 1-\delta_{1}^{\prime}\right)}\end{cases}
$$

where $\beta=n \delta_{1}+m \delta_{2}$ and $\beta^{\prime}=n \delta_{1}^{\prime}+m \delta_{2}^{\prime}$.

Proof First note that the coefficients of $C_{\delta}$ do not depend on $x_{2}$. Hence

$$
d_{\delta}\left(x_{1}, x_{2} ; y_{1}, y_{2}\right)=d_{\delta}\left(x_{1}, 0 ; y_{1}, y_{2}-x_{2}\right)
$$

Without lost of generality one may assume $\left|x_{1}\right| \leq\left|y_{1}\right|$ and so $\left|y_{1}\right| \sim\left|x_{1}\right|+\left|y_{1}\right|$. We adopt this convention throughout the remainder of the proof. Next by the triangle inequality

$$
d_{\delta}\left(x_{1}, 0 ; y_{1}, y_{2}-x_{2}\right) \leq d_{\delta}\left(x_{1}, 0 ; y_{1}, 0\right)+d_{\delta}\left(y_{1}, 0 ; y_{1}, y_{2}-x_{2}\right)
$$

Now we argue that the first term on the right hand side of (32) satisfies the estimate

$$
d_{\delta}\left(x_{1}, 0 ; y_{1}, 0\right) \leq a \frac{\left|x_{1}-y_{1}\right|}{\left(\left|x_{1}\right|+\left|y_{1}\right|\right)^{\left(\delta_{1}, \delta_{1}^{\prime}\right)}}
$$

In order to establish this inequality we distinguish between two cases: $\left|x_{1}-y_{1}\right| \geq\left|y_{1}\right| / 2$ and $\left|x_{1}-y_{1}\right| \leq\left|y_{1}\right| / 2$.

If $\left|x_{1}-y_{1}\right| \geq\left|y_{1}\right| / 2$ then $d_{\delta}\left(x_{1}, 0 ; y_{1}, 0\right) \leq 2 d_{\delta}\left(0,0 ; y_{1}, 0\right)$. But $d_{\delta}\left(0,0 ; y_{1}, 0\right)$ is less than the length of a straight line path from 0 to $y_{1}$. Thus

$$
d_{\delta}\left(x_{1}, 0 ; y_{1}, 0\right) \leq a \frac{\left|y_{1}\right|}{\left|y_{1}\right|^{\left(\delta_{1}, \delta_{1}^{\prime}\right)}} \leq a \frac{\left|x_{1}-y_{1}\right|}{\left(\left|x_{1}\right|+\left|y_{1}\right|\right)^{\left(\delta_{1}, \delta_{1}^{\prime}\right)}} .
$$

If, however, $\left|x_{1}-y_{1}\right| \leq\left|y_{1}\right| / 2$ then we consider the path $\left(x_{1}(t), x_{2}(t)\right)=\left(t x_{1}+(1-t) y_{1}, 0\right)$. Note that $\left|t x_{1}+(1-t) y_{1}\right| \leq\left|y_{1}\right|-t\left|x_{1}-y_{1}\right| \leq\left|y_{2}\right| / 2$ so

$$
d_{\delta}\left(x_{1}, 0 ; y_{1}, 0\right) \leq a \int_{0}^{1} d t\left|x_{1}-y_{1}\right| c_{\delta_{1}, \delta_{1}^{\prime}}\left(\left|y_{1}\right| / 2\right)^{-1 / 2} \sim \frac{\left|x_{1}-y_{1}\right|}{\left(\left|x_{1}\right|+\left|y_{1}\right|\right)^{\left(\delta_{1}, \delta_{1}^{\prime}\right)}}
$$

This completes the bound of the first term on the right hand side of (32).

Next we bound the second term on the right of (32). Specifically we will establish that

$$
d_{\delta}\left(y_{1}, 0 ; y_{1}, y_{2}-x_{2}\right) \leq a \Delta_{\delta}\left(y_{1}, 0 ; y_{1}, x_{2}-y_{2}\right) \sim \Delta_{\delta}\left(x_{1}, x_{2} ; y_{1}, y_{2}\right)
$$

If $\left|x_{2}-y_{2}\right| \leq\left|y_{1}\right|^{\left(\rho, \rho^{\prime}\right)}$ then considering the path $y(t)=\left(y_{1}, t\left(x_{2}-y_{2}\right)\right)$ we find

$$
\begin{aligned}
d_{\delta}\left(y_{1}, 0 ; y_{1}, y_{2}-x_{2}\right) & \leq a \int_{0}^{1} d t\left|x_{2}-y_{2}\right| c_{\delta_{2}, \delta_{2}^{\prime}}\left(\left|y_{1}\right|\right)^{-1 / 2} \\
& \sim\left|x_{2}-y_{2}\right|\left|y_{1}\right|^{\left(-\delta_{2},-\delta_{2}^{\prime}\right)} \sim \Delta_{\delta}\left(x_{1}, x_{2} ; y_{1}, y_{2}\right)
\end{aligned}
$$


If, however, $\left|x_{2}-y_{2}\right| \geq\left|y_{1}\right|^{\left(\rho, \rho^{\prime}\right)}$ we set $\tilde{y}_{1}=\left(y_{1} /\left|y_{1}\right|\right)\left|x_{2}-y_{2}\right|^{\left(1 / \rho, 1 / \rho^{\prime}\right)}$. Then by (34)

$$
d_{\delta}\left(\tilde{y}_{1}, 0 ; \tilde{y}_{1}, y_{2}-x_{2}\right) \leq a\left|x_{2}-y_{2}\right|\left|\tilde{y}_{1}\right|^{\left(-\delta_{2},-\delta_{2}^{\prime}\right)} \sim\left|x_{2}-y_{2}\right|^{\left(1-\gamma, 1-\gamma^{\prime}\right)} \quad .
$$

Therefore

$$
\begin{aligned}
d_{\delta}\left(y_{1}, 0 ; y_{1}, y_{2}-x_{2}\right) & \leq 2 d_{\delta}\left(\tilde{y}_{1}, 0 ; \tilde{y}_{1}, 0\right)+d_{\delta}\left(\tilde{y}_{1}, 0 ; \tilde{y}_{1}, y_{2}-x_{2}\right) \\
& \leq a\left(\left|\tilde{y}_{1}\right|^{\left(1-\delta_{1}, 1-\delta_{1}^{\prime}\right)}+\left|x_{2}-y_{2}\right|^{\left(1-\gamma, 1-\gamma^{\prime}\right)}\right) \\
& \sim\left|x_{2}-y_{2}\right|^{\left(1-\gamma, 1-\gamma^{\prime}\right)} \sim \Delta_{\delta}\left(x_{1}, x_{2} ; y_{1}, y_{2}\right)
\end{aligned}
$$

Combination of these estimates then gives an upper bound

$$
d_{\delta}\left(x_{1}, x_{2} ; y_{1}, y_{2}\right) \leq a D_{\delta}\left(x_{1}, x_{2} ; y_{1}, y_{2}\right)
$$

for all $x, y$. Therefore to complete the proof of equivalence of the distances we have to establish a similar lower bound.

First we argue that

$$
c_{\delta_{1}, \delta_{1}^{\prime}}\left(x_{1}\right)\left(\nabla_{x_{1}} D_{\delta}\left(x_{1}, x_{2} ; y_{1}, y_{2}\right)\right)^{2}+c_{\delta_{2}, \delta_{2}^{\prime}}\left(x_{1}\right)\left(\nabla_{x_{2}} D_{\delta}\left(x_{1}, x_{2} ; y_{1}, y_{2}\right)\right)^{2} \leq a
$$

To establish (35) we first note that $\nabla_{x_{2}}\left(\left|x_{1}-y_{1}\right| /\left(\left|x_{1}\right|+\left|y_{1}\right|\right)^{\left(\delta_{1}, \delta_{1}^{\prime}\right)}\right)=0$. Secondly,

$$
\begin{gathered}
\nabla_{x_{1}} \frac{\left|x_{1}-y_{1}\right|}{\left(\left|x_{1}\right|+\left|y_{1}\right|\right)^{\left(\delta_{1}, \delta_{1}^{\prime}\right)}}=\left(\nabla_{x_{1}}\left|x_{1}-y_{1}\right|\right)\left(\left|x_{1}\right|+\left|y_{1}\right|\right)^{\left(-\delta_{1},-\delta_{1}^{\prime}\right)}+\left|x_{1}-y_{1}\right| \nabla_{x_{1}}\left(\left|x_{1}\right|+\left|y_{1}\right|\right)^{\left(-\delta_{1},-\delta_{1}^{\prime}\right)} \\
\leq\left(\left|x_{1}\right|+\left|y_{1}\right|\right)^{\left(-\delta_{1},-\delta_{1}^{\prime}\right)}+a \frac{\left|x_{1}-y_{1}\right|}{\left(\left|x_{1}\right|+\left|y_{1}\right|\right)^{\left(1+\delta_{1}, 1+\delta_{1}^{\prime}\right)}} \leq a\left(\left|x_{1}\right|+\left|y_{1}\right|\right)^{\left(-\delta_{1},-\delta_{1}^{\prime}\right)}
\end{gathered}
$$

Therefore

$$
c_{\delta_{1}, \delta_{1}^{\prime}}\left(x_{1}\right)\left(\nabla_{x_{1}} \frac{\left|x_{1}-y_{1}\right|}{\left(\left|x_{1}\right|+\left|y_{1}\right|\right)^{\left(\delta_{1}, \delta_{1}^{\prime}\right)}}\right)^{2}+c_{\delta_{2}, \delta_{2}^{\prime}}\left(x_{1}\right)\left(\nabla_{x_{2}} \frac{\left|x_{1}-y_{1}\right|}{\left(\left|x_{1}\right|+\left|y_{1}\right|\right)^{\left(\delta_{1}, \delta_{1}^{\prime}\right)}}\right)^{2} \leq a .
$$

Hence to establish (35) it is enough to show that

$$
c_{\delta_{1}, \delta_{1}^{\prime}}\left(x_{1}\right)\left(\nabla_{x_{1}} \Delta_{\delta}\left(x_{1}, x_{2} ; y_{1}, y_{2}\right)\right)^{2}+c_{\delta_{2}, \delta_{2}^{\prime}}\left(x_{1}\right)\left(\nabla_{x_{2}} \Delta_{\delta}\left(x_{1}, x_{2} ; y_{1}, y_{2}\right)\right)^{2} \leq a
$$

Now

$$
c_{\delta_{1}, \delta_{1}^{\prime}}\left(x_{1}\right)\left(\nabla_{x_{1}}\left|x_{2}-y_{2}\right|^{\left(1-\gamma, 1-\gamma^{\prime}\right)}\right)^{2}=0
$$

and

$$
c_{\delta_{2}, \delta_{2}^{\prime}}\left(x_{1}\right)\left(\nabla_{x_{2}}\left|x_{2}-y_{2}\right|^{\left(1-\gamma, 1-\gamma^{\prime}\right)}\right)^{2} \leq\left(\frac{\left|x_{1}\right|^{\left(\delta_{2}, \delta_{2}^{\prime}\right)}}{\left|x_{2}-y_{2}\right|^{\left(\gamma, \gamma^{\prime}\right)}}\right)^{2} \leq a
$$

for all $\left|x_{2}-y_{2}\right| \geq\left|x_{1}\right|+\left|y_{1}\right|^{\left(\rho, \rho^{\prime}\right)}$. Next

$$
\begin{gathered}
c_{\delta_{1}, \delta_{1}^{\prime}}\left(x_{1}\right)\left(\nabla_{x_{1}} \frac{\left|x_{2}-y_{2}\right|}{\left(\left|x_{1}\right|+\left|y_{1}\right|\right)^{\left(\delta_{2}, \delta_{2}^{\prime}\right)}}\right)^{2} \leq a c_{\delta_{1}, \delta_{1}^{\prime}}\left(x_{1}\right)\left(\frac{\left|x_{2}-y_{2}\right|}{\left(\left|x_{1}\right|+\left|y_{1}\right|\right)^{\left(1+\delta_{2}, 1+\delta_{2}^{\prime}\right)}}\right)^{2} \\
\leq a\left(\frac{\left|x_{2}-y_{2}\right|\left|x_{1}\right|^{\left(\delta_{1}, \delta_{1}^{\prime}\right)}}{\left(\left|x_{1}\right|+\left|y_{1}\right|\right)^{\left(1+\delta_{2}, 1+\delta_{2}^{\prime}\right)}}\right)^{2} \leq a\left(\frac{\left|x_{2}-y_{2}\right|}{\left(\left|x_{1}\right|+\left|y_{1}\right|\right)^{\left(\rho, \rho^{\prime}\right)}}\right)^{2} \leq a
\end{gathered}
$$


for all $\left|x_{2}-y_{2}\right| \leq\left|x_{1}\right|+\left|y_{1}\right|^{\left(\rho, \rho^{\prime}\right)}$. Finally

$$
c_{\delta_{2}, \delta_{2}^{\prime}}\left(x_{2}\right)\left(\nabla_{x_{2}} \frac{\left|x_{2}-y_{2}\right|}{\left(\left|x_{1}\right|+\left|y_{1}\right|\right)^{\left(\delta_{2}, \delta_{2}^{\prime}\right)}}\right)^{2} \leq\left(\frac{\left|x_{1}\right|^{\left(\delta_{2}, \delta_{2}^{\prime}\right)}}{\left(\left|x_{1}\right|+\left|y_{1}\right|\right)^{\left(\delta_{2}, \delta_{2}^{\prime}\right)}}\right)^{2} \leq a .
$$

This completes the verification of (35).

Since $D_{\delta}$ satisfies (35) it follows formally from the definition (4) of the Riemannian distance that $D_{\delta}\left(x_{1}, x_{2} ; y_{1}, y_{2}\right) \leq a d_{\delta}\left(x_{1}, x_{2} ; y_{1}, y_{2}\right)$. One cannot, however, immediately make this deduction since $D_{\delta} \notin W^{1, \infty}\left(\mathbf{R}^{n} \times \mathbf{R}^{m}\right)$. It is, however, a continuous function which is locally Lipschitz differentiable on $\left(\mathbf{R}^{n}-\{0\}\right) \times \mathbf{R}^{m}$. But the distance is not changed if one replaces the space of trial functions $W^{1, \infty}\left(\mathbf{R}^{n} \times \mathbf{R}^{m}\right)$ in the definition (41) by a space of functions which are Lipschitz differentiable on the complement of a closed set of measure zero. This can be deduced by remarking that since we may assume the coefficients are continuous both definitions agree with the shortest path definition of the distance. Therefore $D_{\delta}\left(x_{1}, x_{2} ; y_{1}, y_{2}\right) \leq a d_{\delta}\left(x_{1}, x_{2} ; y_{1}, y_{2}\right)$ and since we have already established the converse inequality one concludes that $D_{\delta}\left(x_{1}, x_{2} ; y_{1}, y_{2}\right) \sim d_{\delta}\left(x_{1}, x_{2} ; y_{1}, y_{2}\right)$.

It remains to prove the volume estimates (31). The proof will be divided into three steps. First we consider small $r$, small compared with $\left|x_{1}\right|$, secondly we consider large $r$ and finally we deal with intermediate values.

Step 1 Assume $c r \leq\left|x_{1}\right|^{\left(1-\delta_{1}, 1-\delta_{1}^{\prime}\right)}$ with $c>1$. In fact we will choose $c \gg 1$ in the course of the proof. We now argue that there are $a_{1}, a_{2} \in \mathbf{R}$ with $0<a_{1}<a_{2}$ such that

$$
\begin{gathered}
\left(x_{1}, x_{2}\right)+\left[-a_{1} r\left|x_{1}\right|^{\left(\delta_{1}, \delta_{1}^{\prime}\right)}, a_{1} r\left|x_{1}\right|^{\left(\delta_{1}, \delta_{1}^{\prime}\right)}\right]^{n} \times\left[-a_{1} r\left|x_{1}\right|^{\left(\delta_{2}, \delta_{2}^{\prime}\right)}, a_{1} r\left|x_{1}\right|^{\left(\delta_{2}, \delta_{2}^{\prime}\right)}\right]^{m} \subset B\left(x_{1}, x_{2} ; r\right) \\
\subset\left(x_{1}, x_{2}\right)+\left[-a_{2} r\left|x_{1}\right|^{\left(\delta_{1}, \delta_{1}^{\prime}\right)}, a_{2} r\left|x_{1}\right|^{\left(\delta_{1}, \delta_{1}^{\prime}\right)}\right]^{n} \times\left[-a_{2} r\left|x_{1}\right|^{\left(\delta_{2}, \delta_{2}^{\prime}\right)}, a_{2} r\left|x_{1}\right|^{\left(\delta_{2}, \delta_{2}^{\prime}\right)}\right]^{m} \cdot
\end{gathered}
$$

Once this is established one has the volume estimates

$$
\left|B\left(x_{1}, x_{2} ; r\right)\right| \sim r^{n+m}\left|x_{1}\right|^{\left(n \delta_{1}+m \delta_{2}, n \delta_{1}^{\prime}+m \delta_{2}^{\prime}\right)}=r^{n+m}\left|x_{1}\right|^{\left(\beta, \beta^{\prime}\right)}
$$

for $r \leq c^{-1}\left|x_{1}\right|^{\left(1-\delta_{1}, 1-\delta_{1}^{\prime}\right)}$

First consider the right hand inclusion of (37). Set $\|x\|=\max _{1 \leq k \leq n}\left|x^{(k)}\right|$ and $\|y\|=$ $\max _{1<l<m}\left|y^{(l)}\right|$ where $x^{(k)}$ and $y^{(l)}$ are the components of $x \in \mathbf{R}^{n}$ and $y \in \mathbf{R}^{m}$, respectively. Thus we have to prove that if $\left(y_{1}, y_{2}\right) \in B\left(x_{1}, x_{2} ; r\right)$ then $\left\|x_{1}-y_{1}\right\| \leq a_{2} r\left|x_{1}\right|^{\left(\delta_{1}, \delta_{1}^{\prime}\right)}$ and $\left\|x_{2}-y_{2}\right\| \leq a_{2} r\left|x_{1}\right|^{\left(\delta_{2}, \delta_{2}^{\prime}\right)}$. But $d_{\delta}\left(x_{1}, x_{2} ; y_{1}, y_{2}\right) \sim D_{\delta}\left(x_{1}, x_{2} ; y_{1}, y_{2}\right)$. Hence if $\left(y_{1}, y_{2}\right) \in$ $B\left(x_{1}, x_{2} ; r\right)$ then $\left\|x_{1}-y_{1}\right\| \leq\left|x_{1}-y_{1}\right| \leq \operatorname{ar}\left(\left|x_{1}\right|+\left|y_{1}\right|\right)^{\left(\delta_{1}, \delta_{1}^{\prime}\right)}$. Therefore

$$
\begin{aligned}
\left\|x_{1}\right\|-\left\|y_{1}\right\| \mid \leq\left\|x_{1}-y_{1}\right\| & \leq a^{\prime} c^{-1}\left(\left|x_{1}\right|+\left|y_{1}\right|\right)^{\left(1-\delta_{1}, 1-\delta_{1}^{\prime}\right)}\left(\left|x_{1}\right|+\left|y_{1}\right|\right)^{\left(\delta_{1}, \delta_{1}^{\prime}\right)} \\
& =a^{\prime} c^{-1}\left(\left|x_{1}\right|+\left|y_{1}\right|\right) \leq a^{\prime} c^{-1}(n+m)\left(\left\|x_{1}\right\|+\left\|y_{1}\right\|\right) .
\end{aligned}
$$

Choosing $c$ large one deduces that $\left\|y_{1}\right\| \sim\left\|x_{1}\right\|$ and $\left|y_{1}\right| \sim\left|x_{1}\right|$. In particular one has

$$
\left\|x_{1}-y_{1}\right\| \leq \operatorname{ar}\left(\left|x_{1}\right|+\left|y_{1}\right|\right)^{\left(\delta_{1}, \delta_{1}^{\prime}\right)} \leq a_{2} r\left|x_{1}\right|^{\left(\delta_{1}, \delta_{1}^{\prime}\right)}
$$

as required.

Secondly, since $\left(y_{1}, y_{2}\right) \in B\left(x_{1}, x_{2} ; r\right)$ one has $\Delta_{\delta}\left(x_{1}, x_{2} ; y_{1}, y_{2}\right) \leq a r$. There are two cases to consider. The first is if $\left|x_{2}-y_{2}\right| \geq\left(\left|x_{1}\right|+\left|y_{1}\right|\right)^{\left(\rho, \rho^{\prime}\right)}$ then $\Delta_{\delta}\left(x_{1}, x_{2} ; y_{1}, y_{2}\right)=$ $\left|x_{2}-y_{2}\right|^{\left(1-\gamma, 1-\gamma^{\prime}\right)}$. Hence

$$
\left|x_{2}-y_{2}\right|^{\left(1-\gamma, 1-\gamma^{\prime}\right)} \leq a r \leq a c^{-1}\left|x_{1}\right|^{\left(1-\delta_{1}, 1-\delta_{1}^{\prime}\right)} \leq a c^{-1}\left(\left|x_{1}\right|+\left|y_{1}\right|\right)^{\left(1-\delta_{1}, 1-\delta_{1}^{\prime}\right)} \quad .
$$


Thus if $c \geq a$ then

$$
\left|x_{2}-y_{2}\right| \leq\left(\left|x_{1}\right|+\left|y_{1}\right|\right)^{\left(\left(1-\delta_{1}\right)(1-\gamma)^{-1},\left(1-\delta_{1}^{\prime}\right)\left(1-\gamma^{\prime}\right)^{-1}\right)}=\left(\left|x_{1}\right|+\left|y_{1}\right|\right)^{\left(\rho, \rho^{\prime}\right)}
$$

which is in contradiction with the assumption $\left|x_{2}-y_{2}\right| \geq\left(\left|x_{1}\right|+\left|y_{1}\right|\right)^{\left(\rho, \rho^{\prime}\right)}$. Therefore one must have $\left|x_{2}-y_{2}\right| \leq\left(\left|x_{1}\right|+\left|y_{1}\right|\right)^{\left(\rho, \rho^{\prime}\right)}$ and $\Delta_{\delta}\left(x_{1}, x_{2} ; y_{1}, y_{2}\right)=\left|x_{2}-y_{2}\right| /\left(\left|x_{1}\right|+\left|y_{1}\right|\right)^{\left(\delta_{2}, \delta_{2}^{\prime}\right)}$. Then, however,

$$
\left\|x_{2}-y_{2}\right\| \leq\left|x_{2}-y_{2}\right| \leq \operatorname{ar}\left(\left|x_{1}\right|+\left|y_{1}\right|\right)^{\left(\delta_{2}, \delta_{2}^{\prime}\right)} \leq a_{2} r\left|x_{1}\right|^{\left(\delta_{2}, \delta_{2}^{\prime}\right)}
$$

because $\left|y_{1}\right| \sim\left|x_{1}\right|$ by the previous argument. Thus the proof of the right hand inclusion of (37) is complete.

Secondly, consider the left hand inclusion of (37). Now we need to prove that if one has $\left\|x_{1}-y_{1}\right\| \leq a_{1} r\left|x_{1}\right|^{\left(\delta_{1}, \delta_{1}^{\prime}\right)}$ and $\left\|x_{2}-y_{2}\right\| \leq a_{1} r\left|x_{1}\right|^{\left(\delta_{2}, \delta_{2}^{\prime}\right)}$ then $\left(y_{1}, y_{2}\right) \in B\left(x_{1}, x_{2} ; r\right)$. But by the first assumption

$$
\left|x_{1}-y_{1}\right| /\left(\left|x_{1}\right|+\left|y_{1}\right|\right)^{\left(\delta_{1}, \delta_{1}^{\prime}\right)} \leq a_{1} n r\left|x_{1}\right|^{\left(\delta_{1}, \delta_{1}^{\prime}\right)} /\left(\left|x_{1}\right|+\left|y_{1}\right|\right)^{\left(\delta_{1}, \delta_{1}^{\prime}\right)} \leq a_{1} n r .
$$

Then by the second assumption

$$
\begin{aligned}
\left|x_{2}-y_{2}\right| \leq a_{1} m r\left|x_{1}\right|^{\left(\delta_{2}, \delta_{2}^{\prime}\right)} & \leq a_{1} m c^{-1}\left|x_{1}\right|^{\left(1+\delta_{2}-\delta_{1}, 1+\delta_{2}^{\prime}-\delta_{1}^{\prime}\right)} \\
& =a_{1} m c^{-1}\left|x_{1}\right|^{\left(\rho, \rho^{\prime}\right)} \leq a_{1} m c^{-1}\left(\left|x_{1}\right|+\left|y_{1}\right|\right)^{\left(\rho, \rho^{\prime}\right)}
\end{aligned}
$$

Hence if $c \geq a_{1} m$ then $\left|x_{2}-y_{2}\right| \leq\left(\left|x_{1}\right|+\left|y_{1}\right|\right)^{\left(\rho, \rho^{\prime}\right)}$. Therefore it follows that $\Delta_{\delta}\left(x_{1}, x_{2} ; y_{1}, y_{2}\right)=$ $\left|x_{2}-y_{2}\right| /\left(\left|x_{1}\right|+\left|y_{1}\right|\right)^{\left(\delta_{2}, \delta_{2}^{\prime}\right)}$. Hence using $\left\|x_{2}-y_{2}\right\| \leq a_{1} r\left|x_{1}\right|^{\left(\delta_{2}, \delta_{2}^{\prime}\right)}$ again one has

$$
\Delta_{\delta}\left(x_{1}, x_{2} ; y_{1}, y_{2}\right) \leq a_{1} m r\left|x_{1}\right|^{\left(\delta_{2}, \delta_{2}^{\prime}\right)} /\left(\left|x_{1}\right|+\left|y_{1}\right|\right)^{\left(\delta_{2}, \delta_{2}^{\prime}\right)} \leq a_{1} m r
$$

and

$$
D_{\delta}\left(x_{1}, x_{2} ; y_{1}, y_{2}\right)=\left|x_{1}-y_{1}\right| /\left(\left|x_{1}\right|+\left|y_{1}\right|\right)^{\left(\delta_{1}, \delta_{1}^{\prime}\right)}+\Delta_{\delta}\left(x_{1}, x_{2} ; y_{1}, y_{2}\right) \leq a_{1}(n+m) r .
$$

Hence if $a_{1}$ is sufficiently small one concludes that $\left(y_{1}, y_{2}\right) \in B\left(x_{1}, x_{2} ; r\right)$.

Step 2 Assume $r / c \geq\left|x_{1}\right|^{\left(1-\delta_{1}, 1-\delta_{1}^{\prime}\right)}$ with $c>1$ where we will again choose $c \gg 1$. We now argue that there are $a_{1}, a_{2} \in \mathbf{R}$ with $0<a_{1}<a_{2}$ such that

$$
\begin{gathered}
\left(0, x_{2}\right)+\left[-a_{1} r^{\left(\sigma, \sigma^{\prime}\right)}, a_{1} r^{\left(\sigma, \sigma^{\prime}\right)}\right]^{n} \times\left[-a_{1} r^{\left(\rho \sigma, \rho^{\prime} \sigma^{\prime}\right)}, a_{1} r^{\left(\rho \sigma, \rho^{\prime} \sigma^{\prime}\right)}\right]^{m} \subset B\left(x_{1}, x_{2} ; r\right) \\
\subset\left(0, x_{2}\right)+\left[-a_{2} r^{\left(\sigma, \sigma^{\prime}\right)}, a_{2} r^{\left(\sigma, \sigma^{\prime}\right)}\right]^{n} \times\left[-a_{2} r^{\left(\rho \sigma, \rho^{\prime} \sigma^{\prime}\right)}, a_{2} r^{\left(\rho \sigma, \rho^{\prime} \sigma^{\prime}\right)}\right]^{m}
\end{gathered}
$$

where $\sigma=\left(1-\delta_{1}\right)^{-1}$ and $\sigma^{\prime}=\left(1-\delta_{1}^{\prime}\right)^{-1}$. These inclusions then yield the volume estimates

$$
\left|B\left(x_{1}, x_{2} ; r\right)\right| \sim r^{\left(n \sigma, n \sigma^{\prime}\right)} r^{\left(m \rho \sigma, m \rho^{\prime} \sigma^{\prime}\right)}=r^{\left(D, D^{\prime}\right)} .
$$

The first step in deducing the inclusions (39) is to observe that if $c$ is sufficiently large then

$$
B\left(0, x_{2} ; r / 2\right) \subseteq B\left(x_{1}, x_{2} ; r\right) \subseteq B\left(x_{1}, x_{2} ; 2 r\right)
$$

and so the proof is effectively reduced to the case $x_{1}=0$. But then the rest of the proof is similar to the argument given in Step 1 but somewhat simpler because of the choice $x_{1}=0$. We omit the details. 
Step 3 It follows from Step 1, and in particular (38), that the volume estimates (31) are valid if $\left|x_{1}\right|^{\left(1-\delta_{1}, 1-\delta_{1}^{\prime}\right)} \geq c r$. Alternatively, it follows from Step 2, and in particular (40), that the estimates are valid if $r \geq c\left|x_{1}\right|^{\left(1-\delta_{1}, 1-\delta_{1}^{\prime}\right)}$. Therefore we now assume that $r \in\left[c^{-1}\left|x_{1}\right|^{\left(1-\delta_{1}, 1-\delta_{1}^{\prime}\right)}, c\left|x_{1}\right|^{\left(1-\delta_{1}, 1-\delta_{1}^{\prime}\right)}\right]$. But then setting $r_{1}=r / c$ and $r_{2}=r c$ one has $B\left(x_{1}, x_{2} ; r_{1}\right) \subset B\left(x_{1}, x_{2} ; r\right) \subset B\left(x_{1}, x_{2} ; r_{2}\right)$. But $r_{1} \leq\left|x_{1}\right|^{\left(1-\delta_{1}, 1-\delta_{1}^{\prime}\right)}$. Hence $B\left(x_{1}, x_{2} ; r_{1}\right) \sim r_{1}^{n+m}\left|x_{1}\right|^{\left(\beta, \beta^{\prime}\right)} \sim r^{n+m}\left|x_{1}\right|^{\left(\beta, \beta^{\prime}\right)}$ by Step 1. Moreover, $r_{2} \geq\left|x_{1}\right|^{\left(1-\delta_{1}, 1-\delta_{1}^{\prime}\right)}$. Hence $B\left(x_{1}, x_{2} ; r_{2}\right) \sim r_{2}^{\left(D, D^{\prime}\right)} \sim r^{\left(D, D^{\prime}\right)}$ by Step 2. Combining these estimates one concludes that

$$
a r^{n+m}\left|x_{1}\right|^{\left(\beta, \beta^{\prime}\right)} \leq\left|B\left(x_{1}, x_{2} ; r\right)\right| \leq a^{\prime} r^{\left(D, D^{\prime}\right)}
$$

for all $r \in\left[c^{-1}\left|x_{1}\right|^{\left(1-\delta_{1}, 1-\delta_{1}^{\prime}\right)}, c\left|x_{1}\right|^{\left(1-\delta_{1}, 1-\delta_{1}^{\prime}\right)}\right]$. But in this range $r^{\left(D, D^{\prime}\right)} \sim a r^{n+m}\left|x_{1}\right|^{\left(\beta, \beta^{\prime}\right)}$. Therefore the volume estimates are established again and the proof of the proposition is complete.

The volume estimates allow one to prove the doubling property and to identify the doubling dimension.

Corollary 5.2 The Riemannian balls $B\left(x_{1}, x_{2} ; r\right)$ associated with the Grušin operator satisfy the doubling property

$$
\left|B\left(x_{1}, x_{2} ; s r\right)\right| \leq a s^{\left(D \vee D^{\prime}\right)}\left|B\left(x_{1}, x_{2} ; r\right)\right|
$$

for all $\left(x_{1}, x_{2}\right) \in \mathbf{R}^{n+m}$ and all $s \geq 1$.

Proof There are three cases to consider.

Case $1 r \leq r s \leq\left|x_{1}\right|^{\left(1-\delta_{1}, 1-\delta_{1}^{\prime}\right)}$. Then the volume estimates of Proposition 5.1 give

$$
\left|B\left(x_{1}, x_{2} ; s r\right)\right| \sim(r s)^{n+m}\left|x_{1}\right|^{\left(\beta, \beta^{\prime}\right)} \sim s^{n+m}\left|B\left(x_{1}, x_{2} ; r\right)\right| .
$$

But $n+m \leq D \vee D^{\prime}$ and $s \geq 1$ so $s^{n+m} \leq s^{\left(D \vee D^{\prime}\right)}$ and the doubling property follows.

Case $2\left|x_{1}\right|^{\left(1-\delta_{1}, 1-\delta_{1}^{\prime}\right)} \leq r \leq r s$. Then the volume estimates give

$$
\left|B\left(x_{1}, x_{2} ; s r\right)\right| \sim(r s)^{\left(D, D^{\prime}\right)} \leq s^{\left(D \vee D^{\prime}\right)} r^{\left(D, D^{\prime}\right)} \sim s^{\left(D \vee D^{\prime}\right)}\left|B\left(x_{1}, x_{2} ; r\right)\right|
$$

and the doubling property is established.

Case $3 r \leq\left|x_{1}\right|^{\left(1-\delta_{1}, 1-\delta_{1}^{\prime}\right)} \leq r s$. Then the volume estimates give

$$
\left|B\left(x_{1}, x_{2} ; s r\right)\right| \sim(r s)^{\left(D, D^{\prime}\right)} \leq s^{\left(D \vee D^{\prime}\right)} r^{\left(D, D^{\prime}\right)} .
$$

But

$$
r^{\left(D, D^{\prime}\right)}=r^{n+m} r^{\left(\beta\left(1-\delta_{1}\right)^{-1}, \beta^{\prime}\left(1-\delta_{1}^{\prime}\right)^{-1}\right)} \leq r^{n+m}\left|x_{1}\right|^{\left(\beta, \beta^{\prime}\right)} \sim\left|B\left(x_{1}, x_{2} ; r\right)\right| .
$$

Combination of these estimates gives the doubling property again.

\section{$6 \quad$ Kernel bounds}

In this section we derive two basic properties of the semigroup kernel $K_{t}$ associated with a general Grušin operator $H$. Initially we show that the kernel conserves probability or, in probabilistic terms, that it is stocastichally complete. Then we establish that it satisfies offdiagonal volume dependent bounds. Subsequently it is possible to apply standard reasoning 
to obtain further more detailed properties such as Gaussian bounds and on diagonal lower bounds. This will be discussed at the end of the section.

First, since the closure of the form $h$ associated with the Grušin operator $H$ is a Dirichlet form the corresponding semigroup $S$ is submarkovian. In particular it extends to a contractive semigroup on $L_{\infty}\left(\mathbf{R}^{n} \times \mathbf{R}^{m}\right)$. In terms of the kernel this means that

$$
0 \leq \operatorname{esssup}_{x \in \mathbf{R}^{n+m}} \int_{\mathbf{R}^{n+m}} d y K_{t}(x ; y) \leq 1
$$

But the particular structure of the operator gives a stronger result.

Theorem 6.1 The semigroup $S$ associated with the Grušin operator on $L_{\infty}\left(\mathbf{R}^{n} \times \mathbf{R}^{m}\right)$ satisfies $S_{t} \mathbb{1}=\mathbb{1}$ for all $t>0$. Hence the kernel satisfies

$$
\int_{\mathbf{R}^{n} \times \mathbf{R}^{m}} d y K_{t}(x ; y)=1
$$

for all $t>0$ almost all $x \in \mathbf{R}^{n+m}$.

Proof Given that the semigroup satisfies the $L_{2}$ off-diagonal bounds of Proposition 4.1 and the volume of the Riemannian balls have polynomial growth, by the estimates of Proposition 5.1, one can prove the theorem by a slight variation of the argument given in Proposition 3.6 of [ERSZ06] but estimating with respect to a Riemannian distance instead of the Euclidean distance. We will, however, give a different variation on the argument which establishes a useful convergence result.

Let $H_{N, \varepsilon}$ be the strongly elliptic approximants, with coefficients $C_{N, \varepsilon}=(C \wedge N I)+\varepsilon I$, to the Grušin operator. Then the semigroups $S_{t}^{(N, \varepsilon)}$ generated by the $H_{N, \varepsilon}$ converge strongly on $L_{2}$ to the semigroup $S_{t}$ in the double limit $N \rightarrow \infty$ followed by $\varepsilon \rightarrow 0$. But the convergence is stronger.

Proposition 6.2 The semigroups $S_{t}^{(N, \varepsilon)}$ converge strongly to $S_{t}$ on each of the $L_{p^{-}}$spaces with $p \in[1, \infty)$ and in the weak $k^{*}$ sense on $L_{\infty}$.

Proof It suffices to prove the convergence on $L_{1}$. Then weak* convergence on $L_{\infty}$ follows by duality and $L_{p}$-convergence for $p \in\langle 1, \infty\rangle$ follows since

$$
\left\|\left(S_{t}^{(N, \varepsilon)}-S_{t}\right) \psi\right\|_{p} \leq\left\|\left(S_{t}^{(N, \varepsilon)}-S_{t}\right) \psi\right\|_{1}^{1 / p}\left(2\|\psi\|_{\infty}\right)^{1-1 / p}
$$

for all $\psi$ in the dense subset $L_{1} \cap L_{\infty}$.

Since the semigroups are contractive it suffices to establish the $L_{1}$-convergence on a subset of $L_{1}$ whose span is dense. In particular it suffices to prove convergence on $L_{1}(A) \cap$ $L_{2}(A)$ for each bounded open subset $A$. Moreover one can restrict to positive functions.

Fix $\varphi_{A} \in L_{1}(A) \cap L_{2}(A)$ and assume $\varphi_{A}$ is positive. Next let $B \supset A$ be a bounded closed set. Then

$$
\begin{aligned}
\left\|\left(S_{t}^{(N, \varepsilon)}-S_{t}\right) \varphi_{A}\right\|_{1} & \leq\left\|\mathbb{1}_{B}\left(S_{t}^{(N, \varepsilon)}-S_{t}\right) \varphi_{A}\right\|_{1}+\left\|\mathbb{1}_{B^{\mathrm{c}}} S_{t}^{(N, \varepsilon)} \varphi_{A}\right\|_{1}+\left\|\mathbb{1}_{B^{\mathrm{c}}} S_{t} \varphi_{A}\right\|_{1} \\
& \leq|B|^{1 / 2}\left\|\left(S_{t}^{(N, \varepsilon)}-S_{t}\right) \varphi_{A}\right\|_{2}+\left(\mathbb{1}_{B^{\mathrm{c}}}, S_{t}^{(N, \varepsilon)} \varphi_{A}\right)+\left(\mathbb{1}_{B^{\mathrm{c}}}, S_{t} \varphi_{A}\right)
\end{aligned}
$$

where we have used the positivity of the semigroups and the functions to express the norms as pairings between $L_{1}$ and $L_{\infty}$. 
Next let $d_{N, \varepsilon}$ denote the Riemannian distance corresponding to the coefficients $C_{N, \varepsilon}$ and $d_{1}$ the distance corresponding to the coefficients $C+I$ then $d_{1} \leq d_{N, \varepsilon}$ for all $N \geq 0$ and all $\varepsilon \in\langle 0,1]$. But it follows from Proposition 4.1 applied to $S_{t}^{(N, \varepsilon)}$ that

$$
\left|\left(\varphi_{C}, S_{t}^{(N, \varepsilon)} \varphi_{A}\right)\right| \leq e^{-d_{N, \varepsilon}(A ; C)^{2}(4 t)^{-1}}\left\|\varphi_{A}\right\|_{2}\left\|\varphi_{C}\right\|_{2} \leq e^{-d_{1}(A ; C)^{2}(4 t)^{-1}}\left\|\varphi_{A}\right\|_{2}\left\|\varphi_{C}\right\|_{2}
$$

for all open sets $C$ and all $\varphi_{C} \in L_{2}(C)$. Now choose $R$ sufficiently large that $A \subseteq B_{1, R}=$ $\left\{x: d_{1}(0 ; x)<R\right\}$ and let $B=\overline{B_{1,2 R}}$. Then one can separate $B^{\mathrm{c}}$ into annuli and make a quadrature estimate, as in the proof of Proposition 3.6 of [ERSZ06], to find

$$
\left(\mathbb{1}_{B^{\mathrm{c}}}, S_{t}^{(N, \varepsilon)} \varphi_{A}\right) \leq \sum_{n \geq 2} e^{-d_{1}\left(B_{1,(n+1) R} \backslash B_{1, n R} ; B_{1, R}\right)^{2}(4 t)^{-1}}\left|B_{1,(n+1) R}\right|^{1 / 2}\left\|\varphi_{A}\right\|_{2}
$$

uniformly for $N \geq 1$ and $\varepsilon \in\langle 0,1]$.

But $d_{1}\left(B_{1,(n+1) R} \backslash B_{1, n R} ; B_{1, R}\right) \geq(n-1) R$ by the triangle inequality. Moreover $H_{1}$, the operator with coefficents $C+I$, is a Grušin operator with $\delta_{1}=0=\delta_{2}$. Hence it follows that $\left|B_{1,(n+1) R}\right|^{1 / 2} \leq a(n R)^{D^{\prime} / 2}$ for all $n \geq 2$ and $R \geq 1$ by Proposition 5.1 . Therefore one obtains an estimate

$$
\left(\mathbb{1}_{B^{\mathrm{c}}}, S_{t}^{(N, \varepsilon)} \varphi_{A}\right) \leq a \sum_{n \geq 2}(n R)^{D^{\prime} / 2} e^{-a^{\prime} n^{2} R^{2} t^{-1}}\left\|\varphi_{A}\right\|_{2}
$$

for all $R \geq 1$, uniform in $N$ and $\varepsilon$. A similar bound is valid for $S_{t}$ by the same reasoning.

Combining these estimates gives

$$
\left\|\left(S_{t}^{(N, \varepsilon)}-S_{t}\right) \varphi_{A}\right\|_{1} \leq\left|B_{1,2 R}\right|^{1 / 2}\left\|\left(S_{t}^{(N, \varepsilon)}-S_{t}\right) \varphi_{A}\right\|_{2}+2 a_{R}\left\|\varphi_{A}\right\|_{2}
$$

where $a_{R} \rightarrow 0$ as $R \rightarrow \infty$ uniformly in $N$ and $\varepsilon$. Therefore

$$
\limsup _{\varepsilon \rightarrow 0, N \rightarrow \infty}\left\|\left(S_{t}^{(N, \varepsilon)}-S_{t}\right) \varphi_{A}\right\|_{1} \leq 2 a_{R}\left\|\varphi_{A}\right\|_{2}
$$

by the $L_{2}$ convergence. Then the $L_{1}$ convergence follows because $a_{R}$ can be made arbitrarily small by choosing $R$ sufficiently large.

Proof of Theorem 6.1 Since the approximants $H_{N, \varepsilon}$ are strongly elliptic $S_{t}^{(N, \varepsilon)} \mathbb{1}=\mathbb{1}$ for all $t>0$. Then by weak* convergence $S_{t} \mathbb{1}=\mathbb{1}$.

Remark 6.3 The proof of Proposition 6.2 and Theorem 6.1 uses very little structure of the Grušin operator. It only requires $L_{2}$ off-diagonal bounds and polynomial volume growth. The first are given for general elliptic operators by Proposition 4.1 and the polynomial growth follows from the Grušin structure.

Next we consider upper bounds on the semigroup kernel.

Theorem 6.4 There is an $a>0$ such that the semigroup kernel $K$ of the Grušin operator $H$ satisfies

$$
0 \leq K_{t}(x ; y) \leq a\left(\left|B\left(x ; t^{1 / 2}\right)\right|\left|B\left(y ; t^{1 / 2}\right)\right|\right)^{-1 / 2}
$$

for all $t>0$ and almost all $x, y \in \mathbf{R}^{n+m}$. 
Since the semigroup $S$ is self-adjoint it follows that the semigroup kernel is positivedefinite. Therefore one formally has $\left|K_{t}(x ; y)\right|^{2} \leq K_{t}(x ; x) K_{t}(y ; y)$ and the estimate apparently reduces to an on-diagonal estimate. But this is only a formal calculation since the kernel is not necessarily continuous and its diagonal value is not necessarily defined. Nevertheless the starting point of the proof is a set-theoretic reduction to an on-diagonal estimate.

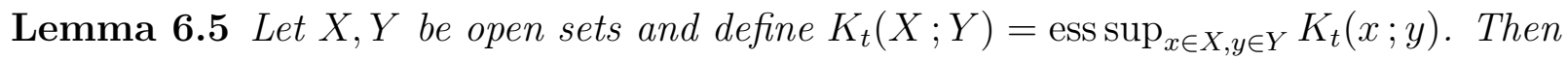

$$
\left|K_{t}(X ; Y)\right|^{2} \leq K_{t}(X ; X) K_{t}(Y ; Y)
$$

Proof First observe that

$$
K_{t}(X ; Y)=\left\|\mathbb{1}_{Y} S_{t} \mathbb{1}_{X}\right\|_{1 \rightarrow \infty} \leq\left\|S_{t / 2} \mathbb{1}_{X}\right\|_{1 \rightarrow 2}\left\|\mathbb{1}_{Y} S_{t / 2}\right\|_{2 \rightarrow \infty}=\left\|\mathbb{1}_{X} S_{t / 2}\right\|_{2 \rightarrow \infty}\left\|\mathbb{1}_{Y} S_{t / 2}\right\|_{2 \rightarrow \infty} .
$$

But if $T$ is bounded from $L_{2}$ to $L_{\infty}$ then $\left(\|T\|_{2 \rightarrow \infty}\right)^{2}=\left\|T T^{*}\right\|_{1 \rightarrow \infty}$. Therefore

$$
\left|K_{t}(X ; Y)\right|^{2} \leq\left\|\mathbb{1}_{X} S_{t} \mathbb{1}_{X}\right\|_{1 \rightarrow \infty}\left\|\mathbb{1}_{Y} S_{t} \mathbb{1}_{Y}\right\|_{1 \rightarrow \infty}=K_{t}(X ; X) K_{t}(Y ; Y)
$$

as required.

Proof of Theorem 6.4 It follows from the lemma that

$$
K_{t}(x ; y) \leq \inf _{X \ni x} K_{t}(X ; X)^{1 / 2} \inf _{Y \ni y} K_{t}(Y ; Y)^{1 / 2}
$$

Thus it suffices to prove that

$$
\inf _{X \ni x} K_{t}(X ; X) \leq a\left|B\left(x ; t^{1 / 2}\right)\right|^{-1}
$$

There are two distinct cases corresponding to the different volume behaviours given by Proposition 5.1 .

First, let $x=\left(x_{1}, x_{2}\right)$ and suppose $\left|x_{1}\right|^{\left(1-\delta_{1}, 1-\delta_{1}^{\prime}\right)} \leq t^{1 / 2}$. Then $\left|B\left(x ; t^{1 / 2}\right)\right| \sim t^{\left(D / 2, D^{\prime} / 2\right)}$ by Proposition [5.1. On the other hand $\left\|S_{t}\right\|_{1 \rightarrow \infty} \leq a t^{\left(-D / 2,-D^{\prime} / 2\right)}$ by Proposition 3.1. Therefore

$$
\inf _{X \ni x} K_{t}(X ; X) \leq\left\|K_{t}\right\|_{\infty}=\left\|S_{t}\right\|_{1 \rightarrow \infty} \leq a t^{\left(-D / 2,-D^{\prime} / 2\right)} \leq a^{\prime}\left|B\left(x ; t^{1 / 2}\right)\right|^{-1} .
$$

Secondly, suppose that $t^{1 / 2} \leq\left|x_{1}\right|^{\left(1-\delta_{1}, 1-\delta_{1}^{\prime}\right)}$. Then $\left|B\left(x ; t^{1 / 2}\right)\right| \sim t^{(n+m) / 2}\left|x_{1}\right|^{\left(\beta, \beta^{\prime}\right)}$ by Proposition 5.1. This case is considerably more difficult to analyze and it is here that we apply the comparison techniques of Section 4.

Set $r=\left|x_{1}\right|$. Let $C$ denote the coefficient matrix of $H$ and choose $a_{n}, a_{m}>0$ such that $C(y) \geq a_{n}\left|y_{1}\right|^{\left(2 \delta_{1}, 2 \delta_{1}^{\prime}\right)} I_{n}+a_{m}\left|y_{1}\right|^{\left(2 \delta_{2}, 2 \delta_{2}^{\prime}\right)} I_{m}$ for all $y=\left(y_{1}, y_{2}\right)$ with $\left|y_{1}\right| \leq r / 2$. Next set $C_{r}(y)=C(y)$ if $\left|y_{1}\right|>r / 2$ and $C_{r}(y)=a_{n} r^{\left(2 \delta_{1}, 2 \delta_{1}^{\prime}\right)} I_{n}+a_{m} r^{\left(2 \delta_{2}, 2 \delta_{2}^{\prime}\right)} I_{m}$ if $\left|y_{1}\right| \leq r / 2$. Then $C_{r} \geq a C$ for a suitable $a>0$. Let $H_{r}$ be the Grušin operator with coefficient matrix $C_{r}$ and set $H_{1}=H_{r}$ and $H_{2}=a H$. Then $H_{1} \geq H_{2}$. Moreover $H_{1} \geq \mu I>0$ for some $\mu=a\left(a_{n} r^{\left(2 \delta_{1}, 2 \delta_{1}^{\prime}\right)} \wedge a_{m} r^{\left(2 \delta_{2}, 2 \delta_{2}^{\prime}\right)}\right)$. Thus the basic assumptions of Theorem 4.6 and Corollary 4.8 are satisfied with this choice. Now $H_{r}$ is a Grušin operator with local parameters $\delta_{1}=0=\delta_{2}$ but with the same global parameters $\delta_{1}^{\prime}, \delta_{2}^{\prime}$ as $H$. Therefore

$$
\left\|S_{t}^{(1,0)}\right\|_{1 \rightarrow \infty} \vee\left\|S_{t}^{(2,0)}\right\|_{1 \rightarrow \infty} \leq V(t)^{-1}
$$


for all $t>0$ where $V(t)=a t^{\left(D / 2, D^{\prime} / 2\right)}$. Since $V$ satisfies the doubling property one may now apply Corollary 4.8. Note that for this application $U=\left\{y:\left|y_{1}\right| \leq r / 2\right\}$ and $d(x ; U) \sim r^{\left(1-\delta_{1}, 1-\delta_{1}^{\prime}\right)}$.

Next we need an improved estimate on the crossnorm $\left\|S_{t}^{(1,0)}\right\|_{1 \rightarrow \infty}$ of the comparison semigroup. Let $\widehat{H}$ denote the constant coefficient operator with coefficients $\widehat{C}_{r}=$ $a_{n} r^{\left(2 \delta_{1}, 2 \delta_{1}^{\prime}\right)} I_{n}+a_{m} r^{\left(2 \delta_{2}, 2 \delta_{2}^{\prime}\right)} I_{m}$. Then $\widehat{H}$ is a Fourier multiplier and the corresponding function $F$ is given by $F\left(p_{1}, p_{2}\right)=a_{n} r^{\left(2 \delta_{1}, 2 \delta_{1}^{\prime}\right)} p_{1}^{2}+a_{m} r^{\left(2 \delta_{2}, 2 \delta_{2}^{\prime}\right)} p_{2}^{2}$. But there is an $a>0$ such that $H_{1} \geq a \widehat{H}$ and we can apply Lemma 2.5 to obtain a uniform bound on $\left\|S_{t}^{(1,0)}\right\|_{1 \rightarrow \infty}$. First, suppose $r=1$ then one immediately has $\left\|S_{t}^{(1,0)}\right\|_{1 \rightarrow \infty} \leq a t^{-(n+m) / 2}$ for all $t>0$. Secondly, the introduction of $r$ corresponds to a dilation of $\mathbf{R}^{n} \times \mathbf{R}^{m}$ with each direction in $\mathbf{R}^{n}$ dilated by $r^{\left(-\delta_{1},-\delta_{1}^{\prime}\right)}$ and each direction in $\mathbf{R}^{m}$ dilated by $r^{\left(-\delta_{2},-\delta_{2}^{\prime}\right)}$. The dilation adds a factor to the crossnorm corresponding to the Jacobian $r^{\left(-n \delta_{1}-m \delta_{2},-n \delta_{1}^{\prime}-m \delta_{2}^{\prime}\right)}=r^{\left(-\beta,-\beta^{\prime}\right)}$ of the dilation. Therefore

$$
\left\|S_{t}^{(1,0)}\right\|_{1 \rightarrow \infty} \leq a^{\prime} t^{-(n+m) / 2} r^{\left(-\beta,-\beta^{\prime}\right)} \leq a^{\prime \prime}\left|B\left(x ; t^{1 / 2}\right)\right|^{-1}
$$

where the last bound follows from the second estimate of Proposition 5.1

Now we may apply Corollary 4.8 with $A=X$. One obtains an estimate

$$
\begin{aligned}
\inf _{X \ni x} K_{t}(X ; X) & =\inf _{X \ni x} K_{t}^{(2,0)}(X ; X) \\
& \leq\left\|S_{t}^{(1,0)}\right\|_{1 \rightarrow \infty}+\inf _{X \ni x} \sup _{y, z \in X}\left|K_{t}^{(1,0)}(y ; z)-K_{t}^{(2,0)}(y ; z)\right| \\
& \leq a\left|B\left(x ; t^{1 / 2}\right)\right|^{-1}+a V\left(t^{2} / \rho^{2}\right)^{-1}\left(\rho^{2} / t\right)^{-1 / 2} e^{-\rho^{2} /(4 t)} \\
& \leq a\left|B\left(x ; t^{1 / 2}\right)\right|^{-1}(1+R(x ; t))
\end{aligned}
$$

where

$$
R(x ; t)=\left|B\left(x ; t^{1 / 2}\right)\right| V\left(t^{2} / \rho^{2}\right)^{-1}\left(\rho^{2} / t\right)^{-1 / 2} e^{-\rho^{2} /(4 t)},
$$

$\rho=d(x ; U) \sim r^{\left(1-\delta_{1}, 1-\delta_{1}^{\prime}\right)}$ and $V(t)=a t^{\left(D / 2, D^{\prime} / 2\right)}$. Thus it suffices to show that $R(x ; t)$ is uniformly bounded for $x, t$ satisfying $t^{1 / 2} \leq r^{\left(1-\delta_{1}, 1-\delta_{1}^{\prime}\right)}$ with $r=\left|x_{1}\right|$. It is necessary to distinguish between three cases.

Case $1 t, r \leq 1$. In this case $t^{1 / 2} \leq r^{1-\delta_{1}} \leq 1$ and $\rho \sim r^{1-\delta_{1}}$. Hence $\rho^{2} / t \sim\left(r^{1-\delta_{1}} t^{-1 / 2}\right)^{2}$ and $t^{2} / \rho^{2} \leq a t$. Therefore, since $t \leq 1$, one must have $V\left(t^{2} / \rho^{2}\right)^{-1} \leq a t^{-D / 2}$. Moreover, $\left|B\left(x ; t^{1 / 2}\right)\right| \sim t^{(n+m) / 2} r^{\beta}$. Then one computes that

$$
\begin{aligned}
R(x ; t) & \leq a t^{(n+m) / 2} r^{\beta} t^{-D / 2}\left(r^{1-\delta_{1}} t^{-1 / 2}\right)^{-1} e^{-a^{\prime}\left(r^{1-\delta_{1}} t^{-1 / 2}\right)^{2}} \\
& =a\left(r^{1-\delta_{1}} t^{-1 / 2}\right)^{-1+\beta\left(1-\delta_{1}\right)^{-1}} e^{-a^{\prime}\left(r^{1-\delta_{1}} t^{-1 / 2}\right)^{2}} .
\end{aligned}
$$

But since $r^{1-\delta_{1}} t^{-1 / 2} \geq 1$ it follows that $R(x ; t)$ is uniformly bounded.

Case $2 t, r \geq 1$. In this case $1 \leq t^{1 / 2} \leq r^{1-\delta_{1}^{\prime}}$ and $\rho \sim r^{1-\delta_{1}^{\prime}}$. Hence $\rho^{2} / t \sim\left(r^{1-\delta_{1}^{\prime}} t^{-1 / 2}\right)^{2}$ and $t^{2} / \rho^{2} \leq a t$. But now $t \geq 1$ and so $V\left(t^{2} / \rho^{2}\right)^{-1} \leq a t^{-D^{\prime} / 2}$. Moreover, $\left|B\left(x ; t^{1 / 2}\right)\right| \sim$ $t^{(n+m) / 2} r^{\beta^{\prime}}$. Then one computes as in Case 1 that

$$
\begin{aligned}
R(x ; t) & \leq a t^{(n+m) / 2} r^{\beta^{\prime}} t^{-D^{\prime} / 2}\left(r^{1-\delta_{1}^{\prime}} t^{-1 / 2}\right)^{-1} e^{-a^{\prime}\left(r^{1-\delta_{1}^{\prime}} t^{-1 / 2}\right)^{2}} \\
& =a\left(r^{1-\delta_{1}^{\prime}} t^{-1 / 2}\right)^{-1+\beta^{\prime}\left(1-\delta_{1}^{\prime}\right)^{-1}} e^{-a^{\prime}\left(r^{1-\delta_{1}^{\prime}} t^{-1 / 2}\right)^{2}} .
\end{aligned}
$$


But since $r^{1-\delta_{1}^{\prime}} t^{-1 / 2} \geq 1$ it again follows that $R(x ; t)$ is uniformly bounded.

Case $3 t \leq 1, r \geq 1$. This is a hybrid case which is rather different to the previous two cases. One again has $\rho \sim r^{1-\delta_{1}^{\prime}}$ and $\rho^{2} / t \sim\left(r^{1-\delta_{1}^{\prime}} t^{-1 / 2}\right)^{2}$. Moreover, $t^{2} / \rho^{2} \leq a t$. But since $t \leq 1$ one has $V\left(t^{2} / \rho^{2}\right)^{-1} \leq a t^{-D / 2}$. In addition $\left|B\left(x ; t^{1 / 2}\right)\right| \sim t^{(n+m) / 2} r^{\beta^{\prime}}$. Therefore one now estimates that

$$
\begin{aligned}
R(x ; t) & \leq a t^{(n+m) / 2} r^{\beta^{\prime}} t^{-D / 2}\left(r^{1-\delta_{1}^{\prime}} t^{-1 / 2}\right)^{-1} e^{-a^{\prime}\left(r^{1-\delta_{1}^{\prime}} t^{-1 / 2}\right)^{2}} \\
& =a r^{\beta^{\prime}}\left(t^{-1 / 2}\right)^{\beta\left(1-\delta_{1}\right)^{-1}}\left(r^{1-\delta_{1}^{\prime}} t^{-1 / 2}\right)^{-1} e^{-a^{\prime}\left(r^{1-\delta_{1}^{\prime}} t^{-1 / 2}\right)^{2}} .
\end{aligned}
$$

But now $r^{1-\delta_{1}^{\prime}} t^{-1 / 2} \geq 1$. Consequently for each $N \geq 1$ there is an $a_{N}$ such that

$$
R(x ; t) \leq a_{N} r^{\beta^{\prime}}\left(t^{-1 / 2}\right)^{\beta\left(1-\delta_{1}\right)^{-1}}\left(r^{1-\delta_{1}^{\prime}} t^{-1 / 2}\right)^{-N}
$$

Choosing $N$ large ensures that this expression is uniformly bounded for all $r \geq 1$ and $t \leq 1$.

The proof of the theorem is now complete.

Theorems 6.1 and 6.4 have a number of standard implications. First one may convert the volume bounds of the latter theorem into Gaussian bounds.

Corollary 6.6 For each $\varepsilon>0$ there is an $a>0$ such that the semigroup kernel $K$ of the Grušin operator satisfies

$$
0 \leq K_{t}(x ; y) \leq a\left(\left|B\left(x ; t^{1 / 2}\right)\right|\left|B\left(y ; t^{1 / 2}\right)\right|\right)^{-1 / 2} e^{-d(x ; y)^{2} /(4(1+\varepsilon) t)}
$$

for all $t>0$ and almost all $x, y \in \mathbf{R}^{n+m}$.

There are several different arguments for passing from on-diagonal kernel bounds to Gaussian bounds (see, for example, the lecture notes of Grigor'yan [Gri99]). One proof of the corollary which is in the spirit of the present paper is given by Theorem 4 of [Sik04]. Note that in the latter reference it is implicitly assumed that the kernel is well-defined on the diagonal but this is not essential. One can argue with open sets and near diagonal estimates as in the proof of the comparison theorem, Theorem 4.6. and in the proof of Theorem 6.4.

It is also a standard argument to pass from the Gaussian bounds of Corollary 6.6 and the conservation property of Theorem 6.1 to on-diagonal lower bounds. But again one has to avoid problems with the definition of the diagonal values.

Corollary 6.7 There is an $a>0$ such that the semigroup kernel $K$ of the Grušin operator satisfies

$$
\inf _{X \ni x}|X|^{-2} \int_{X} d x \int_{X} d y K_{t}(x ; y) \geq a\left|B\left(x ; t^{1 / 2}\right)\right|^{-1}
$$

for all $t>0$ and almost all $x \in \mathbf{R}^{n+m}$ where the average is over open subsets $X$.

Proof At the risk of confusion with the earlier definition we set

$$
K_{t}(X ; Y)=\int_{X} d x \int_{Y} d y K_{t}(x ; y)=\left(\mathbb{1}_{X}, S_{t} \mathbb{1}_{Y}\right)
$$


for each pair of bounded open sets $X, Y$. Then using self-adjointness and the semigroup property one again verifies that

$$
\left|K_{t}(X ; Y)\right|^{2} \leq K_{t}(X ; X) K_{t}(Y ; Y)
$$

Now fix $x$ and $X \ni x$ and let $Y=B\left(x ; R t^{1 / 2}\right)$ for $R>0$. Then

$$
K_{t}(X ; Y)=\int_{X} d x\left(1-\int_{Y^{\mathrm{c}}} d y K_{t}(x ; y)\right)
$$

for all $t>0$ by Theorem 6.1. But now using the Gaussian bounds of Theorem 6.4 and choosing $R$ large one can ensure that

$$
K_{t}(X ; Y) \geq|X| / 2
$$

Then, however, with this choice of $Y$ one has

$$
|X|^{-2} K_{t}(X ; X) \geq\left(4 K_{t}(Y ; Y)\right)^{-1}
$$

But using the bounds of Theorem 6.4 one immediately finds

$$
K_{t}(Y ; Y) \leq a\left(\int_{d(x ; y)<R t^{1 / 2}} d y\left|B\left(y ; t^{1 / 2}\right)\right|^{-1 / 2}\right)^{2}
$$

If $y \in B\left(x ; R t^{1 / 2}\right)$ the doubling property gives

$$
\left|B\left(x ; t^{1 / 2}\right)\right| \leq\left|B\left(y ; d(x ; y)+t^{1 / 2}\right)\right| \leq\left|B\left(y ;(1+R) t^{1 / 2}\right)\right| \leq a(1+R)^{\widetilde{D}}\left|B\left(y ; t^{1 / 2}\right)\right|
$$

with $\widetilde{D}=D \vee D^{\prime}$. In addition

$$
\left|B\left(x ; R t^{1 / 2}\right)\right| \leq a R^{\widetilde{D}}\left|B\left(x ; t^{1 / 2}\right)\right|
$$

Therefore

$$
\begin{aligned}
K_{t}(Y ; Y) & \leq a(1+R)^{2 \widetilde{D}}\left(\int_{d(x ; y)<R t^{1 / 2}} d y\left|B\left(x ; t^{1 / 2}\right)\right|^{-1 / 2}\right)^{2} \\
& \leq a(1+R)^{2 \widetilde{D}}\left|B\left(x ; t^{1 / 2}\right)\right|^{-1}\left|B\left(x ; R t^{1 / 2}\right)\right|^{2} \leq a^{\prime}((1+R) R)^{2 \widetilde{D}}\left|B\left(x ; t^{1 / 2}\right)\right|
\end{aligned}
$$

Combining these estimates gives

$$
|X|^{-2} \int_{X} d x \int_{X} d y K_{t}(x ; y) \geq a\left|B\left(x ; t^{1 / 2}\right)\right|^{-1}
$$

for all bounded open sets $X$ containing $x$ and this gives the statement of the corollary.

Remark 6.8 If the kernel is continuous then the on-diagonal values are well defined and the corollary gives

$$
K_{t}(x ; x)=\inf _{X \ni x}|X|^{-2} \int_{X} d x \int_{X} d y K_{t}(x ; y) \geq a\left|B\left(x ; t^{1 / 2}\right)\right|^{-1}
$$

for all $x \in \mathbf{R}^{n+m}$ and $t>0$. 
The semigroup kernel $K_{t}$ of the Grušin operator $H$ is not necessarily continuous. In particular if $n=1$ and $\delta_{1} \in[1 / 2,1\rangle$ then the kernel is discontinuous. The discontinuity is a direct consequence of the fact established in [ERSZ06] that the action of the corresponding semigroup $S_{t}$ on $L_{2}\left(\mathbf{R} \times \mathbf{R}^{m}\right)$ is not ergodic. If $H_{+}=\left\{\left(x_{1}, x_{2}\right) \in \mathbf{R} \times \mathbf{R}^{m}: x_{1}>0\right\}$ and $H_{-}=\left\{\left(x_{1}, x_{2}\right) \in \mathbf{R} \times \mathbf{R}^{m}: x_{1}<0\right\}$ then $S_{t} L_{2}\left(H_{+}\right) \subseteq L_{2}\left(H_{+}\right)$and $S_{t} L_{2}\left(H_{-}\right) \subseteq L_{2}\left(H_{-}\right)$ for all $t>0$. This is established as follows.

Let $\varphi \in D(h) \subseteq W^{1,2}(\mathbf{R})$ and set $\varphi_{n}=\chi_{n} \varphi$ with $\chi_{n}: \mathbf{R} \rightarrow[0,1]$ defined by

$$
\chi_{n}(x)=\left\{\begin{array}{ll}
0 & \text { if } x \leq-1, \\
-\log |x| / \log n & \text { if } x \in\left\langle-1,-n^{-1}\right\rangle \\
1 & \text { if } x \geq-n^{-1} .
\end{array},\right.
$$

Then one verifies that $\left\|\varphi_{n}-\mathbb{1}_{+} \varphi\right\|_{2} \rightarrow 0$ as $n \rightarrow \infty$, where $\mathbb{1}_{+}$is the indicator function of $\bar{H}_{+}$, and $h\left(\varphi_{n}-\varphi_{m}\right) \rightarrow 0$ as $n, m \rightarrow \infty$. Thus $\mathbb{1}_{+} \varphi \in D(\bar{h})$. (See ERSZ06, Proposition 6.5 and the discussion in Section 4 of RS05. Note that it is crucial for the last limit that $\delta \in[1 / 2,1\rangle$ : the conclusion is not valid for $\delta \in[0,1 / 2\rangle$.) Similarly, replacing $\chi_{n}(x)$ by $\chi_{n}(-x)$ one can conclude that $\mathbb{1}_{-} \varphi \in D(\bar{h})$ with $\mathbb{1}_{-}$the indicator function of $\bar{H}_{-}$. Moreover, $\bar{h}(\varphi)=\bar{h}\left(\mathbb{1}_{+} \varphi\right)+\bar{h}\left(\mathbb{1}_{-} \varphi\right)$. This suffices to deduce that the semigroup leaves the subspaces $L_{2}\left(H_{ \pm}\right)$invariant (see [FOT94, Theorem 1.6.1).

Now one can deduce by contradiction that the kernel has a discontinuity. Suppose the kernel $K_{t}$ of $S_{t}$ is continuous. Then it follows from Remark 6.8 that $K_{t}(x ; x) \geq a_{t}>0$ where $a_{t}=a\left(\sup _{x \in \mathbf{R} \times \mathbf{R}^{m}} \mid B\left(x ; t^{1 / 2} \mid\right)^{-1}\right.$. But since $\left(\varphi, S_{t} \psi\right)=0$ for $\varphi \in L_{2}\left(H_{+}\right)$and $\psi \in L_{2}\left(H_{-}\right)$one must have $K_{t}(x ; y)=0$ for all $x \in H_{+}$and $y \in H_{-}$. But this contradicts the continuity hypothesis.

The separation phenomenon raises the question of boundary conditions on the hypersurface of separation $x_{1}=0$. The closed form $\bar{h}$ has the decomposition $\bar{h}(\varphi)=$ $\bar{h}\left(\mathbb{1}_{+} \varphi\right)+\bar{h}\left(\mathbb{1}_{-} \varphi\right)$ for all $\varphi \in D(h)$, and by closure for all $\varphi \in D(\bar{h})$ Since this is the direct analogue of the decomposition of the form corresponding to the Laplacian with Neumann boundary conditions on the hypersurface $x_{1}=0$ it is tempting to describe the separation in terms of Neumann boundary conditions. But this decomposition is misleading since Neumann and Dirichlet boundary conditions coincide in this case. This can be established by potential theoretic reasoning RS05.

First introduce the form $h_{\text {Dir }}$ by restriction of $h$ to the subspace $D(h) \cap L_{2, c}\left(H_{+} \oplus H_{-}\right)$ where $L_{2, c}(\Omega)$ is defined as the subspace of $L_{2}(\Omega)$ spanned by the functions with compact support. Then $h_{\text {Dir }}$ is closable and its closure $\bar{h}_{\text {Dir }}$ corresponds to the operator $H$ with Dirichlet boundary conditions imposed at the boundary $x_{1}=0$. Moreover, $\bar{h}_{\text {Dir }} \geq \bar{h}$ in the sense of the ordering of forms. But comparison of $\bar{h}_{\text {Dir }}$ and $\bar{h}$ gives a sharp distinction between the weakly degenerate case $\delta_{1} \in[0,1 / 2\rangle$ and the strongly degenerate case $\delta_{1} \in$ $[1 / 2,1\rangle$.

Proposition 6.9 Consider the Grušin operator with $n=1$. If $\delta_{1} \in[0,1 / 2\rangle$ then $\bar{h}_{\text {Dir }}>\bar{h}$ but if $\delta_{1} \in[1 / 2,1\rangle$ then $\bar{h}_{\text {Dir }}=\bar{h}$.

Proof First, observe that $D(h) \cap L_{2, c}\left(H_{+} \oplus H_{-}\right)$is a core of $\bar{h}_{\text {Dir }}$, by definition, and $\bar{h}_{\text {Dir }}=\bar{h}$ in restriction to the core. Secondly, it follows from [RS05, Proposition 3.2, that $D(h) \cap L_{2, c}\left(H_{+} \oplus H_{-}\right)$is a core of $\bar{h}$ if, and only if, $C_{\bar{h}}\left(\left\{x_{1}=0\right\}\right)=0$ where $C_{\bar{h}}(A)$ denotes the capacity, with respect to $\bar{h}$ of the measurable set $A$. Thus $\bar{h}_{\text {Dir }}=\bar{h}$ if, and only if, $C_{\bar{h}}\left(\left\{x_{1}=0\right\}\right)=0$. 
Now suppose $\delta \in[1 / 2,1\rangle$ and define $\xi_{n}$ by $\xi_{n}\left(x_{1}\right)=\chi_{n}\left(x_{1}\right) \wedge \chi_{n}\left(-x_{1}\right)$ where $\chi_{n}$ is given by (45). Then one verifies that $\xi_{n} \in[0,1]$ and $\xi_{n}(x)=1$ for $x \in\left[-n^{-1}, n^{-1}\right]$. Moreover, if $\varphi \in D(h)$ then $\varphi_{n}=\xi_{n} \varphi \in D(h)$ and $h\left(\varphi_{n}\right)+\left\|\varphi_{n}\right\|_{2}^{2} \rightarrow 0$ as $n \rightarrow \infty$. But this means that $C_{\bar{h}}(A)=0$ for each bounded measurable subset of the hypersurface $\left\{x_{1}=0\right\}$. Then it follows by the monotonicity and additivity properties of the capacity (see, for example, [FOT94, Section 2.1, or [BH91, Section 1.8) that $C_{\bar{h}}\left(\left\{x_{1}=0\right\}\right)=0$. This establishes the second statement of the proposition.

Finally suppose $\delta \in[0,1 / 2\rangle$. Then one can find a Fourier multiplier $F$ such that $H \geq F$ and, by Proposition 3.2 , one may choose $F$ such that $1+F\left(p_{1}, p_{2}\right) \geq 1+a\left|p_{1}\right|^{2\left(1-\delta_{1}\right)}$ for some $a>0$ and all $\left(p_{1}, p_{2}\right) \in \mathbf{R} \times \mathbf{R}^{m}$. Then if $U \subset \mathbf{R} \times \mathbf{R}^{m}$ is an open set with $|U|<\infty$ it follows by the calculation at the end of Section 3 of [RS05] that

$$
C_{\bar{h}}(U) \geq|U|^{2}\left(\mathbb{1}_{U},(I+F)^{-1} \mathbb{1}_{U}\right)^{-1} .
$$

Now set $U_{\varepsilon}=\langle-\varepsilon, \varepsilon\rangle \times V$ where $V$ is an open subset of $\mathbf{R}^{m}$. Then calculating as in the proof of Proposition 4.1 of [RS05] one finds

$$
\begin{aligned}
C_{\bar{h}}\left(U_{\varepsilon}\right) & \geq 4 \varepsilon^{2}|V|^{2}\left(4 \varepsilon^{2} \int_{\mathbf{R}^{m}} d p_{2}\left(\tilde{\mathbb{1}}_{V}\left(p_{2}\right)\right)^{2} \int_{\mathbf{R}} d p_{1}\left(1+F\left(p_{1}, p_{2}\right)\right)^{-1}\left(\sin \left(\varepsilon p_{1}\right) /\left(\varepsilon p_{1}\right)\right)^{2}\right)^{-1} \\
& \geq|V|\left(\int_{\mathbf{R}} d p_{1}\left(1+a\left|p_{1}\right|^{2\left(1-\delta_{1}\right)}\right)^{-1}\right)^{-1} \geq a_{\delta_{1}}|V|
\end{aligned}
$$

where $a_{\delta_{1}}>0$. Note that the strict positivity of $a_{\delta_{1}}$ requires $\delta_{1} \in[0,1 / 2\rangle$. Then, however, one must have $C_{\bar{h}}\left(\left\{x_{1}=0\right\}\right)>0$.

The moral of the proposition is that in the strongly degenerate case $\delta \in[1 / 2,1\rangle$ the Dirichlet and Neumann boundary conditions coincide. The separation is a spontaneous effect which is not characterized by a particular choice of boundary conditions.

\section{$7 \quad$ A one dimensional example}

In this section we give a further analysis of the one-dimensional example discussed in ERSZ06, Sections 5 and 6. This example is a special case of the Grušin operator with $n=1, m=0$ and $\delta_{1}^{\prime}=0$. Its structure provides a guide to the anticipated structure of the more interesting examples with $n=1$ and $m \geq 1$.

Let $h(\varphi)=\left(\varphi^{\prime}, c_{\delta} \varphi^{\prime}\right)$ be the form on $L_{2}(\mathbf{R})$ with domain $W^{1,2}(\mathbf{R})$ where $c_{\delta}$ is given by $c_{\delta}(x)=\left(x^{2} /\left(1+x^{2}\right)\right)^{\delta}$ with $\delta>0$. The form is closable and we let $\bar{h}$ denote its closure (relaxation). Let $H$ be the corresponding positive self-adjoint operator on $L_{2}(\mathbf{R})$, $S$ the semigroup generated by $H$ and $K$ the kernel of $S$. All the qualitative features we subsequently derive extend to the semigroups associated with forms $h(\varphi)=\left(\varphi^{\prime}, c \varphi^{\prime}\right)$ with $c \in L_{\infty}(\mathbf{R})$ and $c \sim c_{\delta}$.

The Riemannian distance is now given by $d(x ; y)=\left|\int_{y}^{x} d t c_{\delta}(t)^{-1 / 2}\right|$. If $\delta \in[0,1\rangle$ then $d(\cdot ; \cdot)$ is a genuine distance but if $\delta \geq 1$ then the distance between the left and half right lines is infinite. We concentrate on the case $\delta \in[0,1\rangle$ and comment on the distinctive features of the case $\delta \geq 1$ at the end of the section.

If $\delta \in[0,1\rangle$ the Riemannian ball $B(x ; r)=\{y: d(x ; y)<r\}$ is an interval and the volume is the length of the interval. It is straightforward to estimate this length from the 
explicit form of $c_{\delta}$. One finds $|B(x ; r)| \sim r$ if $|x| \geq 1$ or if $r \geq 1,|B(x ; r)| \sim r^{1 /(1-\delta)}$ if $|x| \leq 1, r \leq 1$ and $d(0 ; x)<r$ and $|B(x ; r)| \sim|x|^{\delta} r$ if $|x| \leq 1, r \leq 1$ and $d(0 ; x) \geq r$. Then $|B|$ satisfies the doubling property (28) with doubling dimension $\widetilde{D}=D=1 /(1-\delta)$. These bounds are all consistent with the general estimates of Proposition 5.1

Moreover, if $B_{+}(x ; r)=B(x ; r) \cap \mathbf{R}_{+}$then $|B(x ; r)| / 2 \leq\left|B_{+}(x ; r)\right| \leq|B(x ; r)|$ for $x \geq 0$. Hence $\left|B_{+}\right|$satisfies similar estimates for $x \geq 0$.

Now we consider bounds on the associated semigroup kernel $K_{t}$. There are two distinct cases $\delta \in[0,1 / 2\rangle$ and $\delta \in[1 / 2,1\rangle$. These correspond to weak and strong degeneracy in the terminology of [ABCF06] and [MV06] who have considered control theory aspects of similar one-dimensional examples.

Case I $\delta \in[0,1 / 2\rangle$. The restriction on $\delta$ means that the degeneracy of the coefficient is relatively mild and the general conclusions of [Tru73] [FKS82 are applicable. But in the present situation one can deduce much more. The Gaussian upper bounds of Corollary 6.6 are valid but there are matching lower bounds.

Proposition 7.1 If $\delta \in[0,1 / 2\rangle$ there are $b, c>0$ such that

$$
K_{t}(x ; y) \geq b\left|B\left(x ; t^{1 / 2}\right)\right|^{-1} e^{-c d(x ; y)^{2} / t}
$$

for all $x, y \in \mathbf{R}$ and $t>0$.

The deduction of the off-diagonal lower bounds requires some additional information on continuity.

Lemma 7.2 If $\delta \in[0,1 / 2\rangle$ then there is an $a>0$ such that

$$
|\varphi(x)-\varphi(y)|^{2} \leq a \frac{d(x ; y)^{2}}{V(x ; y)} h(\varphi)
$$

for all $x, y \in \mathbf{R}$ and all $\varphi \in D(h)$ where $V(x ; y)=|B(x ; d(x ; y))| \vee|B(y ; d(x ; y))|$.

Proof Let $\varphi \in W^{1,2}(\mathbf{R})$. Then

$$
\begin{aligned}
|\varphi(x)-\varphi(y)|^{2} & =\left|\int_{x}^{y} d s \varphi^{\prime}(s)\right|^{2} \\
& \leq\left|\int_{x}^{y} d s c_{\delta}(s)^{-1}\right|\left|\int_{x}^{y} d s c_{\delta}(s) \varphi^{\prime}(s)^{2}\right| \leq\left|\int_{x}^{y} d s c_{\delta}(s)^{-1}\right| h(\varphi) .
\end{aligned}
$$

Now the proof of the lemma follows from the upper bound in the next lemma.

Note that at this point it is essential that $\delta \in[0,1 / 2\rangle$ to ensure that $c_{\delta}^{-1}$ is locally integrable.

Lemma 7.3 If $\delta \in[0,1 / 2\rangle$ then there is an $a>0$ such that

$$
\frac{d(x ; y)^{2}}{|x-y|} \leq\left|\int_{x}^{y} d s c_{\delta}(s)^{-1}\right| \leq a \frac{d(x ; y)^{2}}{V(x ; y)}
$$

for all $x, y \in \mathbf{R}$. 
Proof The left hand bound follows directly from the Cauchy-Schwarz inequality;

$$
d(x ; y)^{2}=\left|\int_{x}^{y} d s c_{\delta}(s)^{-1 / 2}\right|^{2} \leq|x-y|\left|\int_{x}^{y} d s c_{\delta}(s)^{-1}\right| .
$$

The right hand bound uses the volume estimates and follows by treating various different cases.

First set $D(x ; y)=\left|\int_{x}^{y} d s c_{\delta}(s)^{-1}\right|$. Then if $|x-y| \geq 1 / 2$ one has $d(x ; y) \sim|x-y|$ and by similar reasoning $D(x ; y) \sim|x-y|$. Moreover, $V(x ; y) \sim|x-y|$ by the volume estimate with $r \geq 1$. Therefore $D(x ; y) \sim d(x ; y)^{2} / V(x ; y)$. If, however, $x, y \geq 1 / 2$ or $x, y \leq-1 / 2$ then the estimate follows by similar reasoning but using the volume estimate with $|x| \geq 1$. It remains to consider $x, y$ such that $|x-y| \leq 1$. But by symmetry the discussion can be reduced to two cases $0 \leq y<x \leq 1$ and $-1 \leq y<0<x \leq 1$.

Consider the first case. Then $D(x ; y) \sim x^{1-2 \delta}-y^{1-2 \delta}$ and $d(x ; y) \sim x^{1-\delta}-y^{1-\delta}$ by explicit calculation. Moreover, $V(x ; y)=|B(x ; d(x ; y))| \leq a(x-y)$ for some $a \geq 1$. But

$$
\left(x^{1-\delta}-y^{1-\delta}\right)^{2}-\left(x^{1-2 \delta}-y^{1-2 \delta}\right)(x-y)=\left(x^{1 / 2-\delta} y^{1 / 2}-x^{1 / 2} y^{1 / 2-\delta}\right)^{2} \geq 0
$$

This again establishes the required bound.

Finally consider the second case and suppose that $x \geq|y|$. Then $d(x ; y) \sim d(x ; 0) \sim$ $x^{1-\delta}$ and $D(x ; y) \sim D(x ; 0) \sim x^{1-2 \delta}$. Moreover,

$$
V(x ; y)=|B(x ; d(x ; y))| \leq|B(x ; 2 d(x ; 0))| \leq a|B(x ; d(x ; 0))| \leq a^{\prime} x
$$

where the second estimate uses volume doubling. The required bound follows immediately. The case $|y|>x$ is similar with the roles of $x$ and $y$ interchanged.

The bound in Lemma 7.2 is now an immediate consequence of the upper bound of Lemma 7.3. Moreover the Gaussian lower bound in Proposition 7.1]follows directly from the the Gaussian upper bound and the continuity bound of Lemmal7.2. This last implication is, for example, a direct consequence of Theorem 3.1 in Cou03. This theorem is applied with $w=2, p=2$ and $\alpha=1$. Note that the doubling dimension $D=1 /(1-\delta)<\alpha p=2$ because $\delta \in[0,1 / 2\rangle$. Therefore Theorem 3.1 of $\mathrm{Cou} 03$ is indeed applicable. This completes the proof of Proposition 7.1 .

Case $2 \delta \in[1 / 2,1\rangle$. This case corresponds to strong degeneracy in the terms of ABCF06] and [MV06] and describes a quite different situation. It follows from Proposition 6.5 of ERSZ06 that $S_{t} L_{2}\left(\mathbf{R}_{ \pm}\right) \subseteq L_{2}\left(\mathbf{R}_{ \pm}\right)$. Thus the system separates into two ergodic components and the semigroup kernel has the property $K_{t}(x ; y)=0$ for $x<0$ and $y>0$. One can, however, extend the foregoing analysis to the two components. First we prove that the kernel of the semigroup restricted to $L_{2}\left(\mathbf{R}_{+}\right)$is Hölder continuous. Note that the generator of the restriction of the semigroup to $L_{2}\left(\mathbf{R}_{+}\right)$is the operator associated with the closure of the form obtained by restricting $\bar{h}$ to $W^{1,2}\left(\mathbf{R}_{+}\right)=\mathbb{1}_{\mathbf{R}_{+}} W^{1,2}(\mathbf{R})$. Now the continuity proof is by a variation of the usual Sobolev inequalities

$$
|\varphi(x)|^{2} \leq a\left(\left\|\varphi^{\prime}\right\|_{2}^{2}+\|\varphi\|_{2}^{2}\right)
$$

and

$$
|x-y|^{-2 \gamma}|\varphi(x)-\varphi(y)|^{2} \leq a_{\gamma}\left(\left\|\varphi^{\prime}\right\|_{2}^{2}+\|\varphi\|_{2}^{2}\right)
$$


where $\gamma \in\langle 0,1 / 2\rangle$. Fix $\sigma>0$ and let $\psi \in C_{c}^{\infty}(\sigma / 2, \infty)$ with $\psi(x)=1$ for $x>\sigma$. Then

$$
\begin{aligned}
\left\|(\psi \varphi)^{\prime}\right\|_{2}^{2}+\|\psi \varphi\|_{2}^{2} & \leq\left\|\psi^{\prime} \varphi+\varphi^{\prime} \psi\right\|_{2}^{2}+\|\psi \varphi\|_{2}^{2} \leq 2\left\|\psi^{\prime} \varphi\right\|_{2}^{2}+2\left\|\psi \varphi^{\prime}\right\|_{2}^{2}+\|\psi \varphi\|_{2}^{2} \\
& \leq 2\left\|\psi \varphi^{\prime}\right\|_{2}^{2}+(1+4 / \sigma)\|\varphi\|_{2}^{2} \leq 2 c_{\delta}(\sigma / 2)^{-1}\left\|c_{\delta}^{1 / 2} \varphi^{\prime}\right\|_{2}^{2}+(1+4 / \sigma)\|\varphi\|_{2}^{2} \\
& \leq a_{\sigma}\left(h(\varphi)+\|\varphi\|_{2}^{2}\right) .
\end{aligned}
$$

Therefore

$$
|\varphi(x)|^{2}=|(\psi \varphi)(x)|^{2} \leq a_{\sigma}^{\prime}\left(h(\varphi)+\|\varphi\|_{2}^{2}\right)
$$

and

$$
|x-y|^{-2 \gamma}|\varphi(x)-\varphi(y)|^{2} \leq a_{\sigma, \gamma}\left(h(\varphi)+\|\varphi\|_{2}^{2}\right)
$$

for all $x, y \geq \sigma$ and all $\varphi \in W^{1,2}\left(\mathbf{R}_{+}\right)$. These bounds then extend by continuity to the closure of $h$ and are sufficient to deduce that the semigroup kernel $K_{t}$ is uniformly bounded and Hölder continuous on $[\sigma, \infty\rangle \times[\sigma, \infty\rangle$ for each $\sigma>0$. In particular $K_{t}$ is continuous on $\langle 0, \infty\rangle \times\langle 0, \infty\rangle$. But the bounds depend on $\sigma$ and do not give good a priori bounds on $\sup _{x, y>0} K_{t}(x ; y)=\left\|S_{t}\right\|_{1 \rightarrow \infty}$. This can again be accomplished by a Nash inequality argument.

If $h_{+}$temporarily denotes the closed form of the generator of the semigroup on $L_{2}\left(\mathbf{R}_{+}\right)$ and $E \varphi$ denotes the symmetric extension of $\varphi \in L_{2}\left(\mathbf{R}_{+}\right)$to $E \varphi \in L_{2}(\mathbf{R})$ then $\bar{h}(E \varphi)=$ $2 h_{+}(\varphi)$ for all $\varphi \in D\left(h_{+}\right)$. Moreover, $\|E \varphi\|_{2}^{2}=2\|\varphi\|_{2}^{2}$ and $\|E \varphi\|_{1}^{2}=4\|\varphi\|_{1}^{2}$. Therefore the Nash inequalities (7) give similar inequalities

$$
\|\varphi\|_{2}^{2} \leq r^{-2} h_{+}(\varphi)+\pi^{-1} V_{F}(r)\|\varphi\|_{1}^{2}
$$

for all $\varphi \in D\left(h_{+}\right)$. Hence the semigroup restricted to $L_{2}\left(\mathbf{R}_{+}\right)$, or $L_{2}\left(\mathbf{R}_{-}\right)$, again satisfies bounds $\left\|S_{t}\right\|_{1 \rightarrow \infty} \leq a t^{(-1 /(2(1-\delta)),-1 / 2)}$ for all $t>0$. In particular, the kernel $K_{t}$ is uniformly bounded on $\mathbf{R}_{+} \times \mathbf{R}_{+}$, or $\mathbf{R}_{-} \times \mathbf{R}_{-}$, for each $t>0$.

One can then prove the analogues of Corollaries 6.6 and 6.7 on the half-lines.

Proposition 7.4 If $\delta \in[1 / 2,1\rangle$ and $B_{ \pm}(x ; r)=B(x ; r) \cap \mathbf{R}_{ \pm}$then for each $\varepsilon \in\langle 0,1]$ there is an $a>0$ such that

$$
K_{t}(x ; y) \leq a\left|B_{ \pm}\left(x ; t^{1 / 2}\right)\right|^{-1} e^{-d(x ; y)^{2} /(4 t(1+\varepsilon))}
$$

for all $x, y \in \mathbf{R}_{ \pm}$and $t>0$. Moreover, there is $b>0$ such that

$$
K_{t}(x ; x) \geq b\left|B_{ \pm}\left(x ; t^{1 / 2}\right)\right|^{-1}
$$

for all $x \in \mathbf{R}_{ \pm}$and $t>0$.

In the one-dimensional case the statement of Proposition 6.9 can also be described in terms of the vector fields defining $h$ and $h_{\text {Dir }}$.

Let $X=c_{\delta}^{1 / 2} d$, with $d=d / d x$, denote the $C^{\delta}$-vector field acting on $L_{2}(\mathbf{R})$ with domain $D(X)=C_{c}^{\infty}(\mathbf{R})$ and $X_{0}$ the restriction of $X$ to $D\left(X_{0}\right)=C_{c}^{\infty}(\mathbf{R} \backslash\{0\})$. Then $h(\varphi)=\|X \varphi\|_{2}^{2}$ and $h_{\text {Dir }}(\varphi)=\left\|X_{0} \varphi\right\|_{2}^{2}$. It follows straightforwardly that the corresponding positive selfadjoint operators are given by $H=X^{*} \bar{X}$ and $H_{\text {Dir }}=X_{0}^{*} \bar{X}_{0}$. But Proposition 6.9 can now be restated as follows.

Corollary 7.5 If $\delta \in[0,1 / 2\rangle$ then $\bar{X}_{0} \subset \bar{X}$ but if $\delta \in[1 / 2,1\rangle$ then $\bar{X}_{0}=\bar{X}$. 
Although we have restricted attention to the case $\delta \in[0,1\rangle$ the form $h$ is densely defined and closable for all $\delta \geq 0$. But the semigroup and its kernel have very different properties if $\delta \geq 1$. The properties of the distance $d(x ; y)=\left|\int_{y}^{x} d t c_{\delta}(t)^{-1 / 2}\right|$ are also quite different. The distance is finite on the open half line $\langle 0, \infty\rangle$ but since the integral diverges at zero the distance to the origin is at infinity. Now consider points $x, y \in\langle 0,1]$. Then $d(x ; y) \sim|\ln x / y|$ if $\delta=1$ and $d(x ; y) \sim\left|x^{1-\delta}-y^{1-\delta}\right|$ if $\delta>1$. Consider the case $\delta=1$ with the equivalent distance $\tilde{d}(x ; y)=|\ln x / y|$. Let $x_{n} \in\langle 0,1\rangle$ be an arbitrary sequence which converges downward to zero. One may assume $x_{1} \leq e^{-1}$. Then $\tilde{d}\left(x_{n} ; x_{n} e\right)=1=$ $\tilde{d}\left(x_{n} ; x_{n} e^{-1}\right)$ and $\left|B\left(x_{n} ; 1\right)\right|=2 x_{n} \sinh 1$. Therefore $\left|B\left(x_{n} ; 1\right)\right| \rightarrow 0$ as $n \rightarrow \infty$.

Alternatively if $x_{n}=e^{-n}$ with $n \geq 1$ then $B\left(x_{n} ; n / 2\right)=\left\langle e^{-3 n / 2}, e^{-n / 2}\right\rangle$ and $B\left(x_{n} ; n\right)=$ $\left\langle e^{-2 n}, 1\right\rangle$. Therefore $\left|B\left(x_{n} ; n / 2\right)\right|=e^{-n / 2}\left(1-e^{-n}\right) \rightarrow 0$ and $\left|B\left(x_{n} ; n\right)\right|=1-e^{-2 n} \rightarrow 1$ as $n \rightarrow \infty$ so the volume cannot satisfy the volume doubling property.

These divergences allow one to argue that the semigroup kernel is not bounded near the origin.

\section{Applications}

In the foregoing we established that Grušin operators have many important properties in common with strongly elliptic operators; the wave equation has a finite propagation speed, the heat kernel satisfies Gaussian upper bounds and the heat semigroup conserves probability. Then one can readily adapt arguments developed for strongly elliptic operators to obtain further detailed information about the Grušin operators, e.g., information on boundedness of Riesz transforms, spectral multipliers and Bochner-Riesz summability, holomorphic functional calculus, Poincaré inequalities and maximal regularity. We conclude by describing briefly some of these applications to Grušin operators which are a straightforward consequence of general theory and which require no further detailed arguments. It should, however, be emphasized that there are significant differences between the degenerate and the non-degenerate theories related to continuity and positivity properties. In particular the one-dimensional example in Section [ 7 demonstrates that the heat kernel is not necessarily continuous nor strictly positive. Therefore there are limitations to possible extensions of the results of classical analysis to the degenerate case.

\subsection{Boundedness of Riesz transforms}

First we consider boundedness of the Riesz transforms associated with a general Grušin operator $H$ on $L_{p}\left(\mathbf{R}^{n+m}\right)$ for $p \in\langle 1,2]$. The result can be stated in terms of the carré du champ associated with $H$ (see, for example, Section I.4 of [BH91]). Formally the carré du champ is given by

$$
\Gamma_{\psi}=\psi(H \psi)-2^{-1} H \psi^{2}
$$

Note that if $\psi \in C_{c}^{\infty}\left(\mathbf{R}^{n+m}\right)$ then

$$
\Gamma_{\psi}(x)=\sum_{i, j=1}^{n+m} c_{i j}(x)\left(\partial_{i} \psi\right)(x)\left(\partial_{j} \psi\right)(x)
$$

and

$$
\left\|\Gamma_{\psi}\right\|_{1}=\left\|H^{1 / 2} \psi\right\|_{2}
$$


Now one can formulate the result concerning boundedness of the Riesz transform in an analogous manner.

Theorem 8.1 If $H$ is Grušin operator then

$$
\left\|\Gamma_{H^{-1 / 2}}\right\|_{p / 2} \leq\|\psi\|_{p}
$$

for all $\psi \in L^{p}\left(\mathbf{R}^{n+m}\right)$ and all $p \in\langle 1,2]$. In addition the map $\psi \rightarrow \Gamma_{H^{-1 / 2} \psi}^{1 / 2}$ is weak type $(1,1)$, i.e.,

$$
\mid\left\{x \in X:\left|\Gamma_{H^{-1 / 2} \psi}(x)\right|^{1 / 2}>\lambda\right\} \leq a\|\psi\|_{1} / \lambda
$$

for all $\lambda \in \mathbf{R}_{+}$and all $\psi \in L_{1}\left(\mathbf{R}^{n+m}\right)$.

Proof The proof of Theorem 8.1 is a straightforward modification of the proof of Theorem 5 of Sik04]. The assumptions of Theorem 5 of [Sik04 hold in virtue of the property of finite speed of propagation proved in Proposition 4.1 and the kernel bounds (42) of Theorem 6.4.

\subsection{Spectral multipliers}

Each Grušin operator $H$ is positive definite and self-adjoint. Therefore $H$ admits a spectral resolution $E_{H}(\lambda)$ and for any bounded Borel function $F:[0, \infty) \rightarrow \mathbf{C}$ one can define the operator $F(H)$ by

$$
F(H)=\int_{0}^{\infty} d E_{H}(\lambda) F(\lambda) .
$$

It then follows that $F(H)$ is bounded on $L^{2}\left(\mathbf{R}^{n+m}\right)$. Spectral multiplier theorems investigate sufficient conditions on function $F$ which ensure that the operator $F(H)$ extends to a bounded operator on $L_{q}$ for some $q \in[1, \infty]$.

Theorem 8.2 If $H$ is a Grušin operator, $s>\left(D \vee D^{\prime}\right) / 2$ and $F:[0, \infty) \rightarrow \mathbf{C}$ is a bounded Borel function such that

$$
\sup _{t>0}\left\|\eta \delta_{t} F\right\|_{W^{s, \infty}}<\infty
$$

where $\delta_{t} F(\lambda)=F(t \lambda)$ and $\|F\|_{W^{s, p}}=\left\|\left(I-d^{2} / d x^{2}\right)^{s / 2} F\right\|_{L_{p}}$. Then $F(H)$ is weak type $(1,1)$ and bounded on $L_{q}$ for all $q \in\langle 1, \infty\rangle$.

Proof The proof of Theorem 8.2 is a direct consequence of the Gaussian bounds (43) on the heat kernel corresponding to $H$ given by Corollary 6.6] and Theorem 3.1 of [DOS02]. (See also Theorem 3.5 of [CSC95].)

The theory of spectral multipliers is related to and motivated by the study of convergence of the Riesz means or convergence of other eigenfunction expansions of self-adjoint operators. To define the Riesz means of the operator $H$ we set

$$
\sigma_{R}^{s}(\lambda)=\left\{\begin{array}{cc}
(1-\lambda / R)^{s} & \text { for } \lambda \leq R \\
0 & \text { for } \lambda>R
\end{array}\right.
$$

We then define $\sigma_{R}^{s}(H)$ by spectral theory. The operator $\sigma_{R}^{s}(H)$ is the Riesz or the BochnerRiesz mean of order $s$. The basic question in the theory of the Riesz means is to establish the critical exponent for continuity and convergence of the Riesz means. More precisely one wishes to ascertain the optimal range of $s$ for which the Riesz means $\sigma_{R}^{s}(H)$ are uniformly bounded on $L_{1}\left(\mathbf{R}^{n+m}\right)$. A result of this type is given by the following. 
Theorem 8.3 If $H$ is a Grušin operator and $s>\left(D \vee D^{\prime}\right) / 2$ then

$$
\sup _{R>0}\left\|\sigma_{R}^{s}(H)\right\|_{q \rightarrow q} \leq a<\infty
$$

for all $q \in[1, \infty]$. Hence

$$
\lim _{R \rightarrow \infty}\left\|\sigma_{R}^{s}(H) \varphi-\varphi\right\|_{q \rightarrow q}=0
$$

for all $q \in[1, \infty]$ and all $\psi \in L_{q}\left(\mathbf{R}^{n+m}\right)$.

Proof The proof of Theorem 8.3 is again a direct consequence of the Gaussian bounds (43) on the heat kernel corresponding to $H$ and Corollary 6.3 of [DOS02].

Next we consider the implication of the Gaussian bounds on the heat kernel for the holomorphic function calculus of the Grušin operators. First we briefly recall the notion of holomorphic function calculus. For each $\theta>0$ set $\Sigma(\theta)=\{z \in C \backslash\{0\}:|\arg z|<\theta\}$. Let $F$ be a bounded holomorphic function on $\Sigma(\theta)$. By $\|F\|_{\theta, \infty}$ we denote the supremum of $F$ on $\Sigma(\theta)$. The general problem of interest is to find sharp bounds, in terms of $\theta$, of the norm of $F(H)$ as an operator acting on $L_{p}\left(\mathbf{R}^{n+m}\right)$. It is known (see [CDMY96], Theorem 4.10) that these bounds on the holomorphic functional calculus when $\theta$ tends to 0 are related to spectral multiplier theorems for $H$. The following theorem describing holomorphic function calculus for Grušin type operators follows from (43) and Corollary 6.6.

Theorem 8.4 If $H$ is a Grušin operator and $s>\left(D \vee D^{\prime}\right)|1 / p-1 / 2|$ then

$$
\|F(H)\|_{p \rightarrow p} \leq a \theta^{-s}\|F\|_{\theta, \infty}
$$

for all $\theta>0$.

Proof Theorem 8.4 follows from the Gaussian bounds (43) of Corollary 6.6 and Proposition 8.1 of [DOS02].

\subsection{Concluding remarks and comments}

The above statements on the boundedness of the Riesz transforms and the spectral multipliers for Grušin operators are not always optimal. Using the basic estimates of Corollary 6.6 and Proposition 4.1 one can analyze the boundedness of the Riesz transforms for $p>2$.

In the multiplier result discussed above, Theorem 8.2 the critical exponent required for the order of differentiability of the function $F$ is equal to half of the homogeneous dimension $D \vee D^{\prime}$. This is a quite typical situation and for the standard Laplace operator this exponent is optimal. We expect, however, that in many cases it is possible to obtain multiplier results for Grušin operators with critical exponent essentially smaller then the half of the homogeneous dimension $D \vee D^{\prime}$.

It is also possible to obtain a version of the Poincare inequality and Nash type results similar to those discussed in Section [7. But results of this nature require substantial new proofs which we hope to describe elsewhere We conclude by stressing that Corollary 6.6 and Proposition 4.1 provide a sound basis for further analysis of Grušin type operators.

\section{Acknowledgement}

This work was supported by an Australian Research Council (ARC) Discovery Grant DP 0451016. It grew out of an earlier collaboration with Tom ter Elst to whom the authors are indebted for many helpful discussions about degenerate operators. 


\section{References}

[ABCF06] Alabau-Boussouira, F., Cannarsa, P., and Fragnelli, G., Carleman estimates for degenerate parabolic operators with applications to nullcontrollability. J. Evol. Eqs. 6 (2006), 161-204.

[AH05] Ariyoshi, T., and Hino, M., Small-time asymptotic estimates in local Dirichlet spaces. Elec. J. Prob. 10 (2005), 1236-1259.

[Aro67] Aronson, D. G., Bounds for the fundamental solution of a parabolic equation. Bull. Amer. Math. Soc. 73 (1967), 890-896.

[BH91] Bouleau, N., and Hirsch, F., Dirichlet forms and analysis on Wiener space, vol. 14 of de Gruyter Studies in Mathematics. Walter de Gruyter \& Co., Berlin, 1991.

[BR97] Bratteli, O., and Robinson, D. W., Operator algebras and quantum statistical mechanics, vol. 2. Second edition. Springer-Verlag, New York etc., 1997.

[Bra02] Braides, A., Г-convergence for beginners, vol. 22 of Oxford Lecture Series in Mathematics and its Applications. Oxford University Press, Oxford, 2002.

[CDMY96] Cowling, M., Doust, I., McIntosh, A., and Yagi, A., Banach space operators with a bounded $H^{\infty}$ functional calculus. J. Austr. Math. Soc. (Series A) 60 (1996), 51-89.

[CKN84] Caffarelli, L., Kohn, R., and Nirenberg, L., First order interpolation inequalities with weights. Compositio Math. 53 (1984), 259-275.

[CKS87] Carlen, E. A., Kusuoka, S., and Stroock, D. W., Upper bounds for symmetric Markov transition functions. Ann. Inst. Henri Poincaré 23 (1987), $245-287$.

[Cou03] Coulhon, T., Off-diagonal heat kernel lower bounds without Poincaré. J. London Math. Soc. 68 (2003), 795-816.

[CSC95] Coulhon, T., and Saloff-Coste, L., Variétés riemanniennes isométriques à l'infini. Rev. Mat. Iberoamericana 11 (1995), 687-726.

[Dal93] Dal Maso, G., An introduction to $\Gamma$-convergence, vol. 8 of Progress in Nonlinear Differential Equations and their Applications. Birkhäuser Boston Inc., Boston, MA, 1993.

[Dav99] Davies, E. B., A review of Hardy inequalities. In The Maz'ya anniversary collection, Vol. 2 (Rostock, 1998), vol. 110 of Oper. Theory Adv. Appl., 55-67. Birkhäuser, Basel, 1999.

[De 57] De Giongi, E., Sulla differenziabilità e l'analiticità delle estremali degli integrali multipli regolari. Mem. Accad. Sci. Torino cl. Sci. Fis. Mat. Nat. 3 (1957), 25-43. 
[DOS02] Duong, X. T., Ouhabaz, E.-M., and Sikora, A., Plancherel-type estimates and sharp spectral multipliers. J. Funct. Anal. 196 (2002), 443-485.

[ERS06] Elst, A. F. M. ter, Robinson, D. W., and Sikora, A., Small time asymptotics of diffusion processes. J. Evol. Equ. (2006). To appear, arXiv:math.AP/0601350.

[ERSZ06] Elst, A. F. M. ter, Robinson, D. W., Sikora, A., and Zhu, Y., Secondorder operators with degenerate coefficients. J. London Math. Soc. (2006). To appear, arXiv:math.AP/0601307.

[ET76] Ekeland, I., and Temam, R., Convex analysis and variational problems. North-Holland Publishing Co., Amsterdam, 1976.

[FKS82] Fabes, E. B., Kenig, C. E., and Serapioni, R. P., The local regularity of solutions of degenerate elliptic equations. Comm. Part. Diff. Eq. 7 (1982), $77-116$.

[FOT94] Fukushima, M., Oshima, Y., and Takeda, M., Dirichlet forms and symmetric Markov processes, vol. 19 of de Gruyter Studies in Mathematics. Walter de Gruyter \& Co., Berlin, 1994.

[FP83] Fefferman, C., and Phong, D. H., Subelliptic eigenvalue problems. In Conference on harmonic analysis in honor of Antoni Zygmund, Wadsworth Math. Ser., 590-606. Wadsworth, Belmont, CA, 1983.

[FSC86] Fefferman, C. L., and SÁnchez-Calle, A., Fundamental solutions for second order subelliptic operators. Ann. Math. 124 (1986), 247-272.

[Gri92] Grigor'yan, A., The heat equation on noncompact Riemannian manifolds. Math. USSR-Sb. 72, No. 1 (1992), 47-77. Mat. Sb. 182, No. 1, (1991), 55-87.

[Gri99] _ Estimates of heat kernels on Riemannian manifolds. In Spectral theory and geometry (Edinburgh, 1998), vol. 273 of London Math. Soc. Lecture Note Ser., 140-225. Cambridge Univ. Press, Cambridge, 1999.

[Gru70] Grušin, V. V., A certain class of hypoelliptic operators. Mat. Sb. (N.S.) 83 (125) (1970).

[Hör67] Hörmander, L., Hypoelliptic second order differential equations. Acta Math. 119 (1967), 147-171.

[HR03] Hino, M., and RamíREz, J. A., Small-time Gaussian behavior of symmetric diffusion semigroups. Ann. Prob. 31 (2003), 254-1295.

[Jos98] Jost, J., Nonlinear Dirichlet forms. In New directions in Dirichlet forms, vol. 8 of AMS/IP Stud. Adv. Math., 1-47. Amer. Math. Soc., Providence, RI, 1998.

[JSC87] Jerison, D., and SÁnChez-CALle, A., Subelliptic, second order differential operators. In Berenstein, C. A., ed., Complex analysis III, Lecture Notes in Mathematics 1277. Springer-Verlag, Berlin etc., 1987, 46-77. 
[Kat80] Kato, T., Perturbation theory for linear operators. Second edition, Grundlehren der mathematischen Wissenschaften 132. Springer-Verlag, Berlin etc., 1980.

[Mos94] Mosco, U., Composite media and asymptotic Dirichlet forms. J. Funct. Anal. 123 (1994), 368-421.

[MV06] Martinez, P., and Vancostenoble, J., Carleman estimates for onedimensional degenerate heat equations. J. Evol. Eqs. 6 (2006), 325-362.

[Nas58] NAsh, J., Continuity of solutions of parabolic and elliptic equations. Amer. J. Math. 80 (1958), 931-954.

[Ouh05] Ouhabaz, E.-M., Analysis of heat equations on domains, vol. 31 of London Mathematical Society Monographs Series. Princeton University Press, Princeton, NJ, 2005.

[Rob91] Robinson, D. W., Elliptic operators and Lie groups. Oxford Mathematical Monographs. Oxford University Press, Oxford etc., 1991.

[RS05] Robinson, D. W., and Sikora, A. Degenerate elliptic operators: capacity, flux and separation, 2005. arXiv:math.AP/0601351.

[SC95] SAloff-Coste, L., Parabolic Harnack inequality for divergence-form secondorder differential operators. Potential Anal. 4 (1995), 429-467.

[SC02] _ Aspects of Sobolev-type inequalities. London Math. Soc. Lect. Note Series 289. Cambridge University Press, Cambridge, 2002.

[Sik96] SikorA, A., Sharp pointwise estimates on heat kernels. Quart. J. Math. Oxford 47 (1996), 371-382.

[Sik04] _ Riesz transform, Gaussian bounds and the method of wave equation. Math. Z. 247 (2004), 643-662.

[Sim78] Simon, B., A canonical decomposition for quadratic forms with applications to monotone convergence theorems. J. Funct. Anal. 28 (1978), 377-385.

[Str67] Strichartz, R. S., Multipliers on fractional Sobolev spaces. J. Math. Mech. 16 (1967), 1031-1060.

[SW06] SAwyer, E. T., and Wheeden, R. L., Hölder continuity of weak solutions to subelliptic equations with rough coefficients. Mem. Amer. Math. Soc. 180, No. 847 (2006).

[Tru73] Trudinger, N. S., Linear elliptic operators with measurable coefficients. Ann. Scuola Norm. Sup. Pisa 27 (1973), 265-308.

[Xu92] XU, C. J., Regularity for quasilinear second order subelliptic equations. Comm. Pure Appl. Math. 45 (1992), 77-96. 